\title{
Modulation of neuronal excitability in the cognitive control network by electrical stimulation
}

\author{
Dissertation
}

for the award of the degree

Doctor rerum naturalium

of the Georg-August-Universität Göttingen

within the doctoral program Neurosciences

of the Georg-August University School of Science (GAUSS)

submitted by

Albert Lehr

from Berlin, Germany

Göttingen 2020 


\title{
Members of the Thesis Advisory Committee and the Examination Board
}

\author{
Prof. Dr. Andrea Antal \\ Clinic for Clinical Neurophysiology, University Medical Center Göttingen \\ Prof. Dr. Susann Boretius \\ Functional Imaging Laboratory, German Primate Center \\ Arezoo Pooresmaeili, MD, Ph.D. \\ Perception and Cognition Group, European Neuroscience Institute

\section{Further members of the Examination Board}

\section{Dr. Peter Dechent}

Cognitive Neurology, University Medical Center Göttingen

Dr. Roberto Goya-Maldonado

Laboratory of Systems Neuroscience and Imaging in Psychiatry, University Medical Center Göttingen

Prof. Dr. Melanie Wilke

Cognitive Neurology, University Medical Center Göttingen

Date of submisssion:

2nd of April, 2020

Date of oral examination:

14th of May, 2020 


\section{Contents}

$\begin{array}{lll}\text { Frontpage } & \text { I }\end{array}$

Contents

List of Abbreviations $\quad$ VII

$\begin{array}{lll}\text { Abstract } & \text { IX }\end{array}$

1 General introduction $\quad 1$

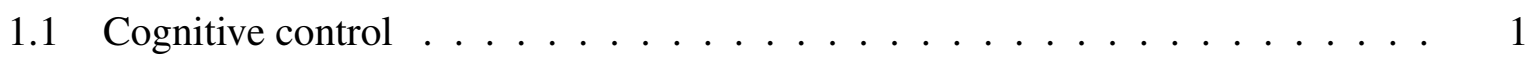

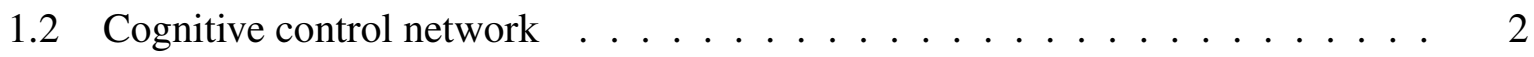

1.2.1 Dorsolateral prefrontal cortex ................ 4

1.2.2 Dorsal anterior cingulate cortex .............. 5

1.2.3 Different roles of dACC and DLPFC . . . . . . . . . 8

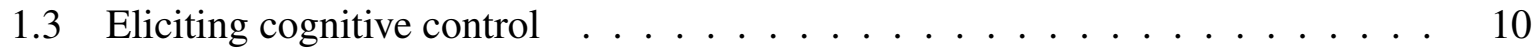

1.3.1 Stroop task ......................... 10

1.3.2 Instrumental learning and Pavlovian biases . . . . . . . . . 11

1.4 Neural oscillations . . . . . . . . . . . . . . . . . 12

1.5 Transcranial electrical stimulation . . . . . . . . . . . . . . 14

1.5.1 Technical limitations of tES . . . . . . . . . . 15

1.6 Objectives and structure of this dissertation $\ldots \ldots \ldots$

2 Modulation of conflict processing by theta range tACS over the dorsolateral pre$\begin{array}{lr}\text { frontal cortex } & 18\end{array}$

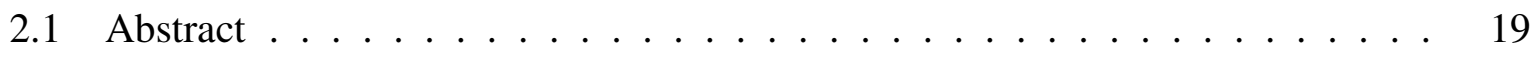

2.2 Introduction . . . . . . . . . . . . . . . . . . 19

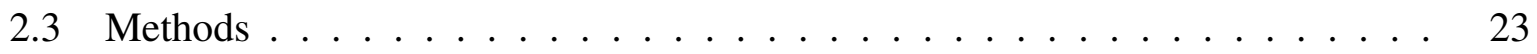

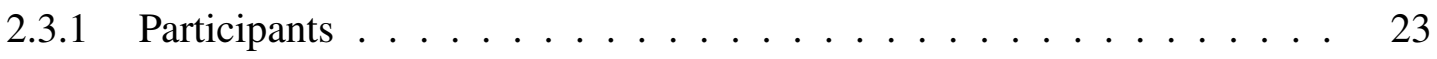

2.3.2 Experimental protocol ................... 23 
2.3 .3 Task . . . . . . . . . . . . . . . . . . . . . 24

2.3.4 Transcranial alternating current stimulation . . . . . . . . . 25

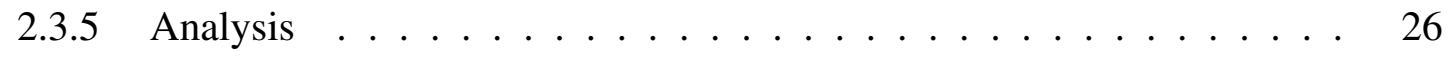

2.3.5.1 Generalized linear mixed models . . . . . . . . . . 26

2.3.5.2 Fitting drift diffusion models for conflict tasks . . . . . . 27

2.3.5.3 Arousal and sleep . . . . . . . . . . . . . . 28

2.4 Results . . . . . . . . . . . . . . . . . . . . . . . . . . . . . . 29

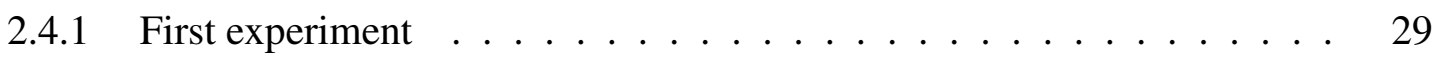

$2.4 .2 \quad$ Second experiment $\ldots \ldots \ldots \ldots \ldots \ldots \ldots \ldots \ldots \ldots$

2.4.3 Joint analysis of both datasets $\ldots \ldots \ldots \ldots \ldots \ldots$

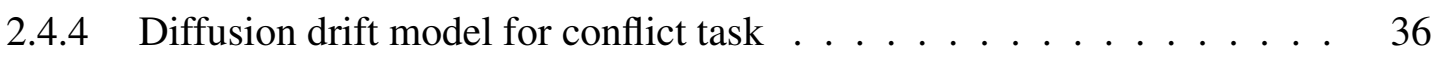

2.4 .5 Arousal and sleep . . . . . . . . . . . . . . . 36

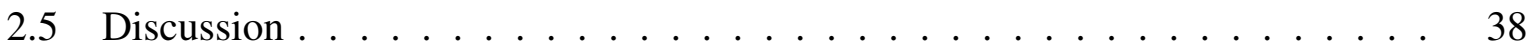

2.5.1 Electrophysiology of the Stroop task . . . . . . . . . . . . 38

2.5.2 Outlook and clinical relevance $\ldots \ldots \ldots \ldots \ldots \ldots$

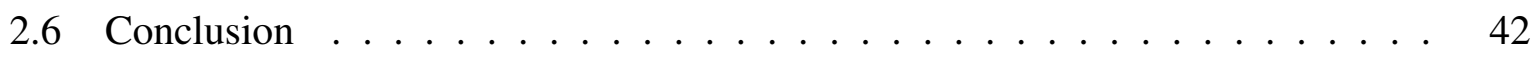

2.7 Supplementary material $\ldots \ldots \ldots \ldots \ldots$

3 Theta transcranial alternating current stimulation decreases conflict effect in Stroop task $\quad 46$

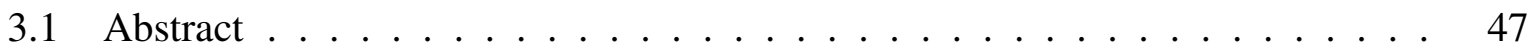

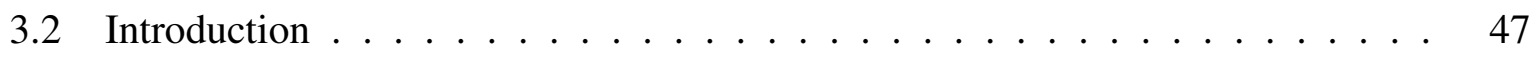

3.3 Methods ........................... 50

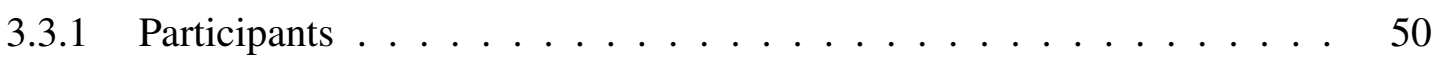

3.3 .2 Experimental protocol $\ldots \ldots \ldots \ldots \ldots \ldots \ldots$

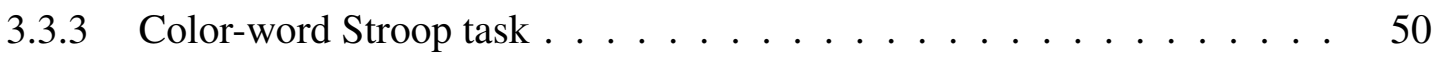

3.3.4 Transcranial electrical stimulation $\ldots \ldots \ldots \ldots \ldots \ldots$

3.3 .5 EEG recording $\ldots \ldots \ldots \ldots \ldots \ldots \ldots \ldots \ldots \ldots$

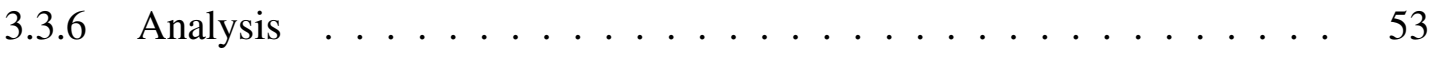


3.3.6.1 Sleep and arousal . . . . . . . . . . . . . 54

3.3.6.2 Generalized linear mixed models for behavioral data . . . . . 54

3.3.6.3 Time-frequency analysis . . . . . . . . . . 55

3.3.6.4 Drift diffusion models for conflict tasks . . . . . . . . . . 56

3.4 Results . . . . . . . . . . . . . . . . . . . . . . . 57

3.4 .1 Behavioral results . . . . . . . . . . . . . . . 57

3.4.1.1 Stimulation phase $\ldots \ldots \ldots \ldots \ldots$

3.4.1.2 Before and after stimulation phase $\ldots \ldots \ldots \ldots$

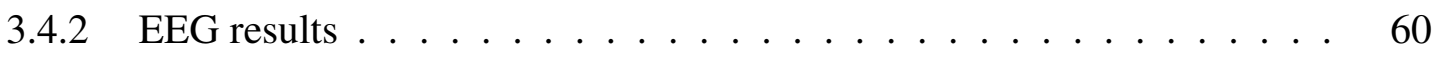

3.4.3 Drift diffusion model for conflict tasks . . . . . . . . . . . . . . 63

3.5 Discussion . . . . . . . . . . . . . . . . . . . 63

3.6 Supplementary material . . . . . . . . . . . . . . . . . 67

4 Theta-gamma cross-frequency transcranial alternating current stimulation over $\begin{array}{lr}\text { the trough impairs cognitive control } & 68\end{array}$

$4.1 \quad$ Abstract . . . . . . . . . . . . . . . . . . . . . . . . . . . 69

4.2 Introduction . . . . . . . . . . . . . . . . . . . . . . 69

4.3 Methods . . . . . . . . . . . . . . . . . . . . . . . 71

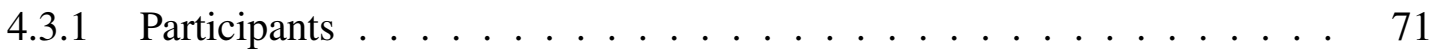

4.3.2 Ethic statement and data availability . . . . . . . . . . . 72

4.3 .3 Experimental design $\ldots \ldots \ldots \ldots \ldots \ldots$

4.3.4 Behavioral Paradigm . . . . . . . . . . . . . . . . . 72

4.3.5 Transcranial alternating current stimulation $\ldots \ldots \ldots \ldots \ldots$

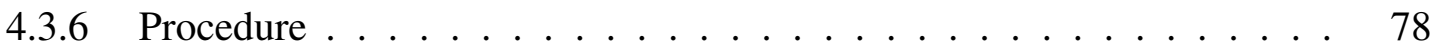

4.3.7 Statistical analysis . . . . . . . . . . . . . . . . . 79

4.3.8 Computational modelling $\ldots \ldots \ldots \ldots \ldots$

4.4 Results . . . . . . . . . . . . . . . . . . . . . . 80

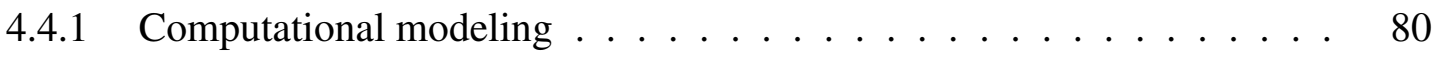

4.4 .2 Accuracy and learning $\ldots \ldots \ldots \ldots \ldots \ldots$ 
4.4 .3 Average accuracy .................... 83

4.4.4 Learning rate analysis .................... 85

4.4.5 Perceptual adverse effects ................... 86

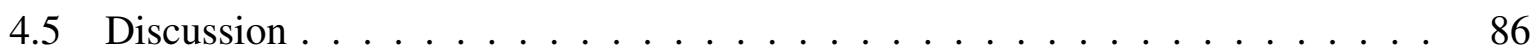

4.6 Supplemental information . . . . . . . . . . . . . . . . . 90

5 General Discussion $\quad 96$

5.1 DLPFC and dACC causally underlie cognitive control . . . . . . . . . . . 96

5.2 Scientific limitations of tES . . . . . . . . . . . . . . 98

5.3 General conclusions and perspectives . . . . . . . . . . . . . 100

$\begin{array}{ll}\text { References } & 101\end{array}$

$\begin{array}{ll}\text { Bibliography } & 101\end{array}$

$\begin{array}{ll}\text { List of Figures } & \text { X }\end{array}$

$\begin{array}{lll}\text { List of Tables } & \text { XI }\end{array}$

A Acknowledgments $\quad$ XII

B Declaration of Contribution $\quad$ XIII

$\begin{array}{ll}\text { C Curriculum Vitae } & \text { XIV }\end{array}$ 


\section{List of Abbreviations}

\begin{tabular}{|c|c|}
\hline AIC & Anterior insular cortex \\
\hline $\mathrm{CCN}$ & Cognitive control network \\
\hline $\mathrm{CCT}$ & Congruency of the current trial \\
\hline $\mathrm{CFC}$ & Cross-frequency coupling \\
\hline $\mathrm{CPT}$ & Congruency of the preceding trial \\
\hline $\mathrm{dACC}$ & Dorsal anterior cingulate cortex \\
\hline DDM & Drift diffusion model \\
\hline DLPFC & Dorsolateral prefrontal cortex \\
\hline DMC & Drift diffusion model for conflict tasks \\
\hline dPMC & Dorsal premotor cortex \\
\hline EEG & Electroencephalography \\
\hline EVC & Expected value of control \\
\hline fMRI & Funtional magnetic resonance imaging \\
\hline HDI & Highest-density interval \\
\hline HMC & Hamiltonian Monte-Carlo \\
\hline IFJ & Inferior frontal junction \\
\hline LOOIC & Leave-one-out information criterion \\
\hline PFC & Prefrontal cortex \\
\hline PPC & Posterior parietal cortex \\
\hline pSMA & Pre-supplementary motor area \\
\hline RL & Reinforcement learning \\
\hline
\end{tabular}


List of Abbreviations

ROI Region of interest

RT Response time(s)

tACS Transcranial alternating current stimulation

tDCS Transcranial direct current stimulation

tES Transcranial electrical stimulation

tRNS Transcranial random noise stimulation 


\section{Abstract}

Cognitive control is the ability to behave advantageously while ignoring distracting stimuli or automated behaviors. Dorsolateral prefrontal cortex (DLFPC) and dorsal anterior cingulate cortex (dACC) are the main hubs of the cognitive control network which is active during cognitive control. The strength of their interaction was shown to correlate to neural oscillations in midtheta range that modify gamma range oscillations. In this thesis, the roles of DLPFC and dACC in the emergence of cognitive control are investigated.

Transcranial electrical stimulation induces changes in brain activity by externally inducing currents. A subform, transcranial alternating current stimulation (tACS), is thought to entrain neural oscillations, which lead to the propagation of activity throughout networks.

In the first two experiments a total of 22 participants performed a color-word Stroop task which induces cognitive control. Concurrent tACS in the mid-theta range and targeted to the DLPFC reduced the Stroop effect, a indirect measure of cognitive control.

In the next experiment 24 participants performed a similar color-word Stroop task while concurrently receiving, among others, mid-theta range tACS. The stimulation was here targeted to the dACC. Again, Stroop effect was reduced. Task-based electroencephalography measured before and after stimulation did not concur with the reduced Stroop effect found during stimulation.

In a fourth experiment 24 participants performed a conflict-inducing instrumental-learning task during which they received cross-frequency tACS that nested gamma oscillations into theta oscillations. Again, the stimulation was targeted to the dACC. Reduction in cognitive control occurred phase-specifically, i.e. when gamma oscillations occurred during the trough of theta oscillations.

In summary, the causal role of theta and gamma oscillations within the cognitive control network for the emergence of cognitive control is affirmed. 


\section{General introduction}

\subsection{Cognitive control}

Higher cognitive functions are widely regarded as setting humans apart from all other living animals. While neuroscience has shown few animals possess similar cognitive functions, the flexibility, self-regulation and self-reflection of humans' higher cognition is unmatched (Premack, 2007). This cognition allows wilful choosing and planning of how to achieve intentional goals. Attaining chosen goals necessitates selecting appropriate behaviors. Also, the constant implementation of and the outcomes of these behaviors have to be supervised until the goal was either attained or abandoned.

Executive functions are the cognitive processes that control other brain processes. As such they are necessary for selecting and controlling the implementation of complex goal-directed behavior. The goal has to be maintained in working memory during implementation, although distracting external stimuli are perceived constantly. These external stimuli might elicit behaviors that are not in line with the attainment of the chosen goals. Therefore, these unhelpful behaviors have to be actively inhibited to allow the correct behavior to continue. Thus, these more basic executive functions (selection and implementation of goals) need to be engaged and supported by cognitive control.

Cognitive control entails the ability to orchestrate behaviors and cognitive processes based on task demands, while simultaneously suppressing inappropriate habitual actions or interference by competing stimuli (Miller and Cohen, 2001). This corresponds to the executive functions information updating and monitoring as well as inhibition of automatic responses as defined in the seminal paper by Miyake et al. (2000). According to Botvinick et al. (2001), cognitive control is the ability of the brain to adjust perception, bias responses and maintain contextual information depending on specific task requirements (Niendam et al., 2012).

Cognitive control describes the adaption of cognitive processes to current task demands. By exerting top-down control, information processing is biased towards attending to currently important stimuli and to actively maintaining task rules for selecting appropriate behavior (Chein and Schneider, 2005). Brain regions responsible for cognitive control are active early during practice in many tasks. As they become less active upon further practice, apparently the need for cognitive control declines with practice (Chein and Schneider, 2005). This begs the ques- 
tion why cognitive control is not implemented perpetually in order to always maintain the best possible task performance and to allow for well-planned instead of habituated responses.

Exerting cognitive control is effortful and thereby carries an intrinsic subjective cost (Kool et al., 2010). Similarly to physical effort, mental effort is minimized where possible. A multitude of studies have shown humans to rely on automatic, not on effortful mental calculations whenever possible, perhaps best described in Kahneman (2011). Humans are biased against top-down control requiring tasks, even delaying task goals or foregoing rewards if the mental effort is too burdensome compared to the reward (Dixon and Christoff, 2012; Kool et al., 2010; Westbrook et al., 2013). Also, different intensities of cognitive control (as required by task difficulty) exist and correlate with mental costs. This implies a cost-benefit trade-off between the advantage of improved performance and the mental cost of implementing a specific level of cognitive control (Dixon and Christoff, 2012; Kool et al., 2010). Therefore, cognitive control has to be implemented just when the expected improvement/reward outweighs the mental effort and has to be minimized to the levels still sufficient for optimal performance.

\subsection{Cognitive control network}

The term cognitive control network $(\mathrm{CCN})$ was coined as a multitude of cognitive control tasks consistently activate the same cortical areas (e.g. feedback-based Go/No-Go task Cavanagh et al. (2013); Stroop task Bench et al. (1993)). These areas act as a domain-general control system (Dosenbach et al., 2006; Chein and Schneider, 2005). These tasks include conflicting or novel information and require instructions to be converted to stimulus-response associations for optimal task performance (Cole and Schneider, 2007). Selection of the correct behaviors depends on these stimulus-response associations and structurally on the prefrontal cortex (PFC, Miller (2000)). The active cortical areas include the dorsolateral prefrontal cortex (DLPFC) and the dorsal anterior cingulate cortex in conjunction with the pre-supplementary motor area (dACC / pSMA), which are both prefrontal target areas for the stimulation experiments described in this dissertation (Cole and Schneider, 2007). Additionally, the posterior parietal cortex (PPC), the dorsal premotor cortex (dPMC), the anterior insular cortex (AIC) and the inferior frontal junction (IFJ) complete the CCN according to Cole and Schneider (2007) (see Figure 1). These brain areas form a functional network as their activity showed higher correlation among themselves than with other brain regions both in rest and in task performance. However, the CCN was 
also connected strongly to other cortical regions, which agrees with the purported role of master regulator of many cortical processes (Cole and Schneider, 2007). In a meta-analysis of 193 functional neuroimaging studies including 2,832 healthy participants prefrontal areas as DLPFC, anterior cingulate cortex, frontopolar cortex and orbitofrontal cortex and also the superior and inferior parietal cortices constituted the CCN (Niendam et al., 2012). Furthermore, a wide array of regions were activated concurrently based on specific task demands.

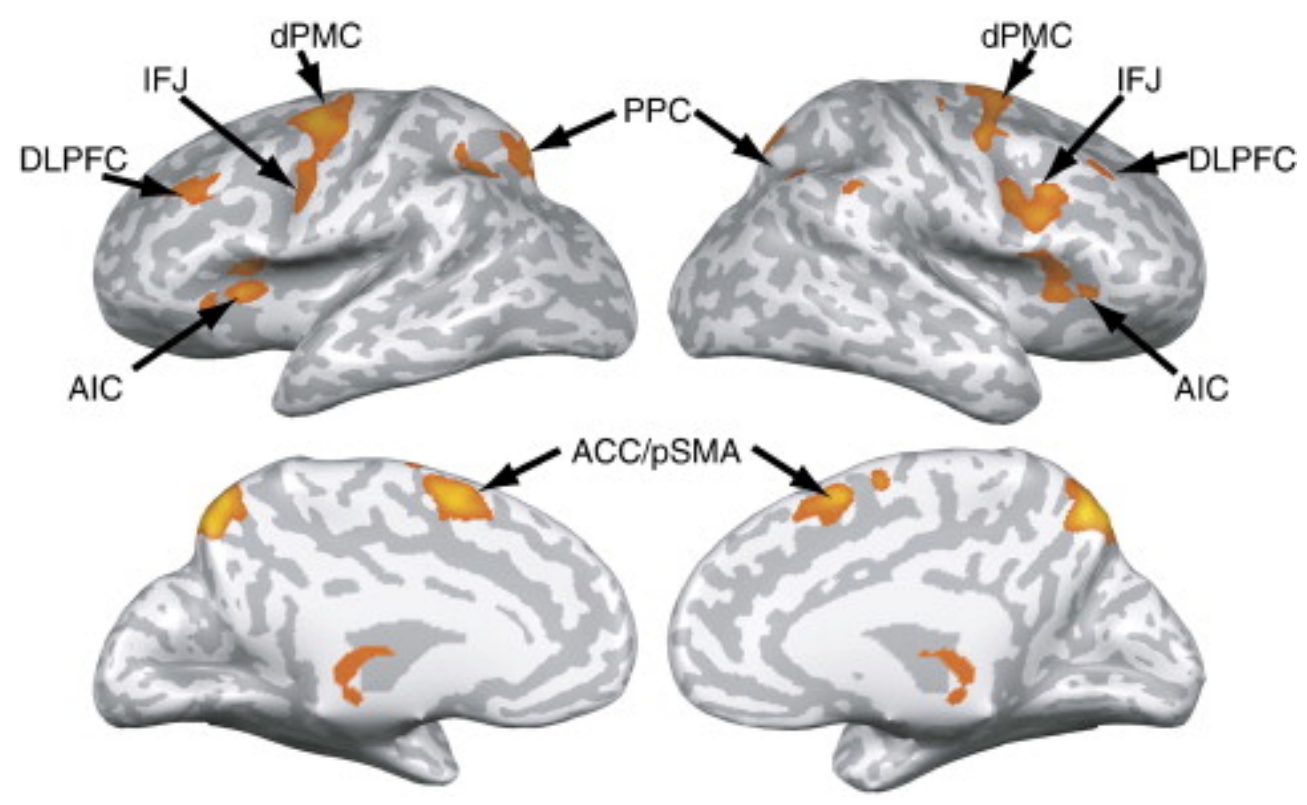

Figure 1: These six locations constitute the cognitive control network according to Cole and Schneider (2007). DLPFC - dorsolateral prefrontal cortex; IFJ - inferior frontal junction; AIC - anterior insular cortex; dPMC - dorsal premotor cortex; PPC - posterior parietal cortex; ACC/pSMA - anterior cingulate cortex and pre-supplementary motor area. Modified from Figure 2, Cole and Schneider (2007).

While not entirely overlapping, Cole and Schneider (2007) and Niendam et al. (2012) agree on the central importance of the frontal cortex for the CCN. Specifically, DLPFC and dACC are often implicated as taking over key roles in cognitive control (Curtis and D’Esposito, 2003; Kerns et al., 2004). Their activity correlates highly throughout a plethora of task demands, suggesting their tight communication to be essential and necessary for many underlying functions (Duncan and Owen, 2000). Also, the dACC in conjunction with the AIC has been shown to causally induce activation of the DLPFC and PPC during tasks while simultaneously deactivating the default mode network, which is active during task-free resting state (Sridharan et al., 2008). 


\subsubsection{Dorsolateral prefrontal cortex}

The DLPFC lies in the middle frontal gyrus (see Figure 2). It corresponds to Brodmann areas 46 and includes the lateral part of Brodmann area 9 (Mtui et al., 2015). The DLPFC is defined by its widespread connections. Anatomically closely linked structures include the anterior and posterior cingulate cortex, premotor areas, the PPC, superior temporal cortex, retrosplenial cortex and the neocerebellum (Collins et al., 2001).

The DLPFC is, however, better defined functionally than anatomically. It is activated as the terminal part of the dorsal visual stream that underlies spatial processing (Goodale and Milner, 1992; Mtui et al., 2015). According to O'Reilly (2010), this stream mainly underpinned by the parietal cortex is responsible for the perception for action planning and is therefore called the 'how' stream. The DLPFC is a control system of the dorsal stream and maps stimuli to responses. Where a task with easy-to-discriminate stimuli and complex rules engaged the dorsal stream and therefore the DLPFC, another task with hard-to-discriminate stimuli but easy rules activated the ventral stream ('what' stream) and its control region, the ventrolateral prefrontal cortex (Nagel et al., 2008). Thus, this stream is responsible for maintaining rules that match stimuli to correct behaviors.

In cognitive control tasks, the DLPFC has been shown to be activated strongly when visual working memory is sustained for eight seconds (Barch et al., 1997). However, DLPFC activity declined when visual working memory was sustained for thirty-two seconds, while the purportedly subordinated areas dPMC, AIC, IFJ and lateral PPC maintained activity throughout the full period (Cole and Schneider, 2007). This result was corroborated by single-unit recordings in primates. Lateral PPC activity was constant during working memory demands, but DLPFC activity declined (Quintana and Fuster, 1999). DLPFC activity seems to ensure correct task performance by converting stimuli into stimuli-behavior associations, which are transferred to subordinate regions during sustained task performance (Cole and Schneider, 2007). Processing in the posterior parietal cortex, which is involved in the execution of control-demanding behaviors, is then biased appropriately (Shenhav et al., 2013).

DLPFC activity correlates with response conflict (Laird et al., 2005). Thus, DLPFC activity is heightened during high-conflict (incongruent) trials in Stroop task and correlates with behavioral performance (Silton et al., 2010). This suggests a crucial role of the DLPFC in implementing cognitive control and thereby adjusting behavior appropriately (Mansouri et al., 2009). However, 
the DLPFC contributes to conflict-induced behavioral adjustment alongside the dACC (Mansouri et al., 2007). These adjustments become necessary when conflicting rules or responses have to be resolved before proper response selection, as is the case in the Wisconsin Card Sorting Test or the Stroop task (Grant and Berg, 1948; MacLeod and MacDonald, 2000). In the former task, conflict-induced behavioral adjustments were distinguished by artificial lesions in the DLPFC of macaque monkeys, while they persisted after lesions to the dACC (Mansouri et al., 2007). More generally, pathological DLPFC activity has been related to reduced attention and motivation (Miller and Cummings, 2017). Minor DLPFC lesions led to disinterest in one's surroundings and to less motivation for actions for oneself or others (Miller and Cummings, 2017).

\subsubsection{Dorsal anterior cingulate cortex}

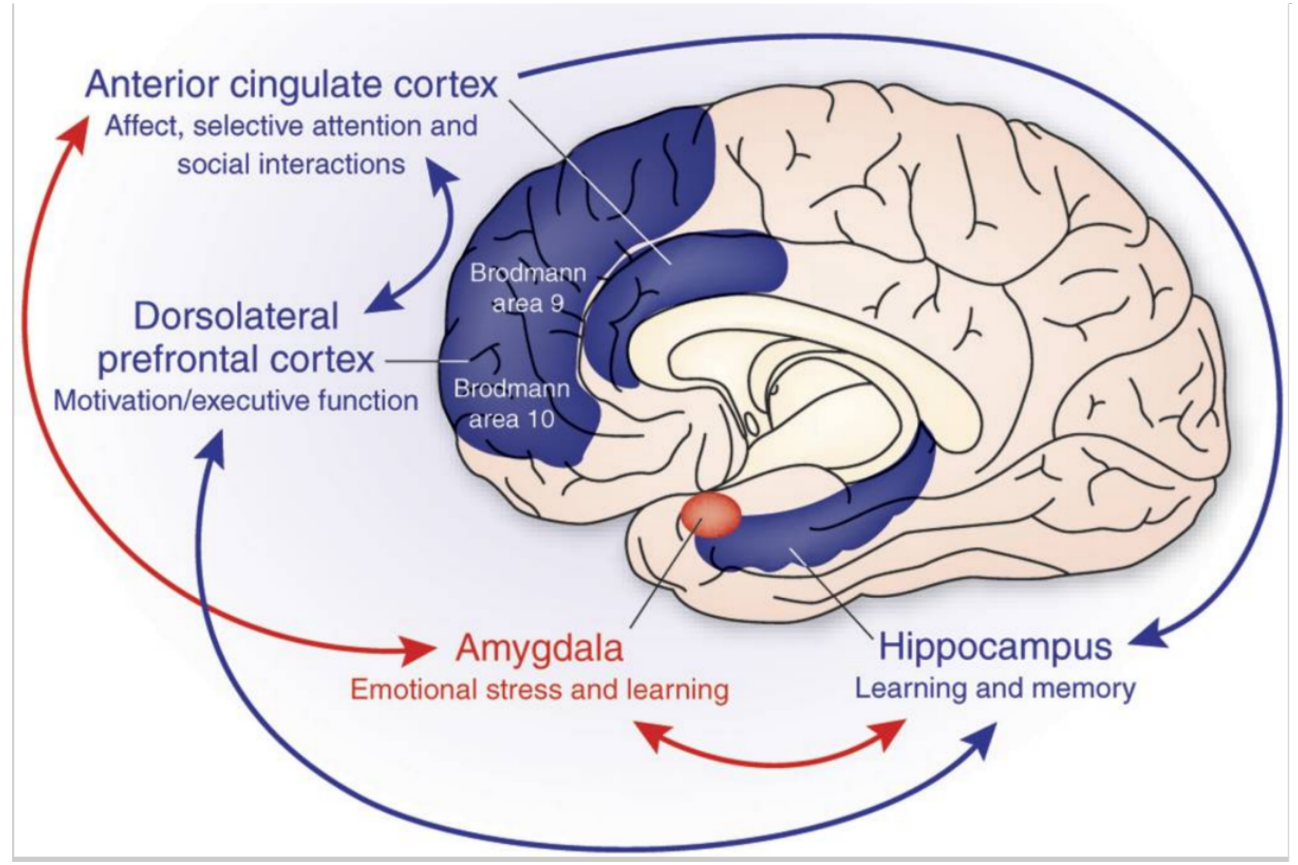

Figure 2: The anatomical location and the functions of the dorsolateral prefrontal cortex and the anterior cingulate cortex and their relation to emotional processing (Amygdala) and memory/learning (Hippocampus) is shown. Modified from Figure 1, Benes (2010).

Anatomically, dACC is located dorsally of the genu of the corpus callosum and consists of Brodmann areas 24 and the dorsal part of 32 (see Figure 2; Heilbronner and Hayden (2016)). As 
part of the cingulate gyrus, the AACC lies between the ventral ACC and the posterior cingulate cortex. It was originally coined as midcingulate cortex before the term dACC came into broader use (Vogt and Gabriel, 1993; Vogt et al., 2005). The cingulate gyrus itself is the cortical area of the telencephalon farthest from the brain's surface as it lies enclosed by the frontal, temporal and parietal lobe of the brain.

The dACC has been associated with the functions emotions, cognitive control and motor control (Morecraft and Van Hoesen, 1998; Paus, 2001; Rushworth et al., 2011). For each distinctive function, multiple connections to major brain areas have been associated (Heilbronner and Hayden, 2016). Projections to and from the amygdala, hypothalamus, ventromedial prefrontal cortex, insula, ventral striatum suggest a role in emotion, while motor control functions are implied by projections to and from the pre- and motor cortex, and the spinal cord. Similarly, cognitive control is supported by connections to and from the DLPFC ('How' stream), ventrolateral prefrontal cortex ('What' stream), orbitofrontal cortex and the parietal cortex, as reviewed in Heilbronner and Hayden (2016). In cognitive control tasks, dACC activity correlates positively with task demands (Barch et al., 1997)

Damage to the dACC causes apathy, less voluntary actions due to insufficient energy or inability, emotional instability and akinetic mutism (Paus, 2001). While these pathologies do not allow one to infer one specific function for the dACC, specifically performance in tasks inducing cognitive control is reduced. For instance responses are slower, errors persevere longer and the current response speed is not adapted based on the difficulty of the preceding trials as in healthy subjects (Stuss and Alexander, 2007; Stuss, 2011). The latter is coined 'conflict adaptation effect' (also Gratton effect) and consists of a high-conflict/incongruent trial (I) in a conflict task influencing the response times of the next trial (Egner, 2007; Gratton et al., 1992). If this next trial is also high-conflict/incongruent (iI, the capitalized letter indicates the second trial), the response speed is higher than normal during high-conflict, while low-conflict/congruent trials (C) are slowed (iC; Figure 3). This is commonly described as a result of the exertion of cognitive control (Egner, 2007). 


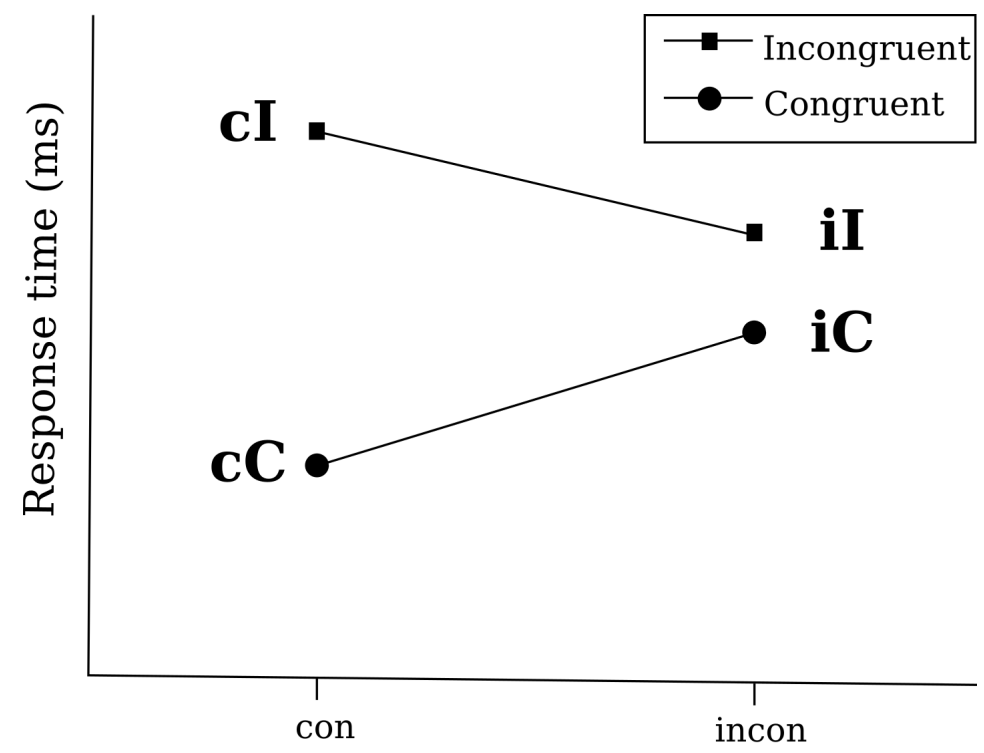

Congruency of previous trial

Figure 3: The Conflict adaptation effect first described by Gratton et al. (1992) is illustrated. Response times change according to the congruence of trials. The response time difference between congruent and incongruent trials (Stroop effect) is modified by the congruency of the previous trial (Conflict adaptation effects).

Sheth et al. (2012) provide evidence that dACC lesions causally extinguish the conflict adaptation effect, which was also observed in patients about to undergo cingulotomy. After the operation the conflict adaptation effect vanished. Similarly, older adults with decline in the white matter bundle projecting to and from the dACC showed impaired performance in control-demanding tasks (Metzler-Baddeley et al., 2012). Gläscher et al. (2012) analyzed the performance of human patients with focal brain lesions in control-demanding tasks and found rostral dACC lesions to be associated with the worst performance. Strategic adjustments are hindered by dACC lesions as reduced rewards or delayed but greater rewards do not change behavior (Rushworth et al., 2003; Kennerley et al., 2006). All this evidence suggests a causal role of the dACC in cognitive control.

A study of four patients with lesions in the dACC cast doubt on the causal importance of the dACC. The patients showed unimpaired conflict adaptation to differing degrees of response conflicts in both Go/NoGo and Stroop tasks (Fellows and Farah, 2005). Similar lesions in animals, however, impaired optimal decision-making about action implementation in animals (Kennerley et al., 2006). As these animals failed to update the values attached to actions during both positive and negative reinforcement, a broader function of the $\mathrm{dACC}$ in estimation of reward certainty is 
possible (Mansouri et al., 2009). Overall, the often correlative nature of evidence does not allow final conclusions about the dACC function within the $\mathrm{CCN}$.

\subsubsection{Different roles of dACC and DLPFC}

The wide-ranging connections to functionally diverse structures of the brain and the activation of the dACC in a multitude of tasks, have led to many theories about the role of dACC within the CCN. In their seminal paper Botvinick et al. (2001) proposed the conflict-monitoring role of dACC. Herein, it monitors information processing and detects conflicts, which it then signals to the DLPFC, which in turn implements control. Ample evidence is given for this theory, for instance: Higher activation in dACC during high-conflict than low- or no-conflict trials (MacDonald et al., 2000; van Veen et al., 2001). This theory predicts differing roles within conflict adaptation for the $\mathrm{dACC}$ and the DLPFC. It stipulates that regions active during all incongruent trials are monitoring for and detecting conflicts and that regions active only during iI exert cognitive control.

Indeed, during conflict trials the degree of dACC activity predicted the magnitude of behavioral adjustment and the level of activity in the DLPFC in subsequent trials (Kerns et al., 2004; Egner and Hirsch, 2005a,b; Liston et al., 2006). Also, DLPFC activity increased in iI and correlated with behavioral adjustment whereas dACC activity was low (Egner and Hirsch, 2005a).

Shenhav et al. (2013) have expanded this theory and proposed a single function underlying all the functional connections and wide-ranging pattern of activation of the dACC. The dACC might integrate the expected value of implementing unhabituated behaviors against the cost of exerting cognitive control. The dACC then signals the DLPFC if and how strongly to implement control in order to maximize the expected value of control (EVC). Shenhav et al. (2013) presumes the $\mathrm{dACC}$ to receive all information regarding the benefits of a behavior (reward predictions) and to be solely responsible for deciding the type and the degree of control (e.g. which task to focus on, ratio between automatic and controlled processing) to be implemented. The DLPFC then implements the cognitive control together with subordinated structures. Thus, valuation happens in functionally preceding brain structures, the dACC then monitors for current demands to increase cognitive control according to cost-benefit analysis, specifies the cognitive control and the DLPFC regulates the cognitive control (Shenhav et al., 2013). The AIC is responsible for primary valuation and conveys affective salience, which affects motivation during frequent 
coactivation with the dACC (Bush et al., 2000; Ullsperger et al., 2010; Medford and Critchley, 2010). These affective signals can induce changes to internal salient states and overt behaviors mediated via the dACC. Also, these salient states can themselves trigger cognitive control if they interfere with performance. Additionally, ventromedial prefrontal cortex, striatum, amygdala and the dopaminergic midbrain signal value and/or prediction errors (Shenhav et al., 2013).

Clearly, increased cogntive control will improve performance, but as mentioned above it comes with a (mental) cost. Therefore, the dACC in order for the EVC to be optimized, the exerted cognitive control should be as low as possible while still reaping most benefits of increased cognitive control. Greater activity in the dACC predicted decreased later activity in a brain structure encoding monetary rewards, the nucleus accumbens (Botvinick et al., 2009). Also, participants in behavioral studies did indeed often adopt nearly optimal response behaviors after changes in task demands (Holmes and Cohen, 2014). It is widely reported that dACC activity correlates with cognitive demands of tasks (Botvinick, 2007; Venkatraman and Huettel, 2012; Paus et al., 1998). This EVC optimization has to be adjusted according to current task demands and discounts future rewards based on the degree of cognitive control needed.

The dACC is sensitive to both conflicts in processing and overt errors (Yeung et al., 2004). According to the EVC theory the dACC appraises all events which might signal a need for control, potentially even task-irrelevant events. The dACC reacts to a wide range of negative outcomes as pain (Shackman et al., 2011), (likelihood of) errors in task performance (Brown and Braver, 2005), monetary loss (Blair et al., 2006) and also less-than-anticipated monetary rewards (Holroyd et al., 2004). The latter shows the dACC to code loss context-dependent and not absolute. These findings have been corroborated in intracranial recordings in animals (Niki and Watanabe, 1979; Ito, 2003; Gabriel and Orona, 1982). Even conflict itself might not only signal increased task difficulty but also be a negative-valued outcome (Botvinick, 2007; Fritz and Dreisbach, 2013; Hirsh et al., 2012).

The dACC also encodes reward information, e.g. the reward intensity (Amiez et al., 2006), unchosen rewards (Blanchard and Hayden, 2014) or progress towards defined goals (Shidara and Richmond, 2002). The dACC is thereby responsible for persistence until goal completion (Chudasama et al., 2013). It is associated with choosing long-term benefits over instant gratification (Peters and Büchel, 2010) and, possibly, the will to endure against adversities (Parvizi et al., 2013). 
In summary, the EVC theory assumes the dACC to be a central hub of the brain network responsible for cogntive control. Rushworth et al. (2011) argue that actions are linked to outcomes by the dACC and these guide behavior by encoding consequences to actions. Alexander and Brown (2011) expand this by stating that the dACC compares actual to previously predicted associations between stimuli and action outcomes (Alexander and Brown, 2011). Activation of dACC is more closely correlated with action-outcome than with stimulus-outcome Luk and Wallis (2013). Other theories however assumes that the dACC not only monitors action outcome/EVC and triggers cognitive control but also implements the latter. The dACC and AIC might be key for task-set maintenance, thus the origins of cognitive control (Dosenbach et al., 2006, 2008). Both AIC and dACC combine phasic activation to salient stimuli but more constant activation associated with maintenance of task-performance. However, this would oppose the role of dACC and AIC as mediators between task-positive and task-free states (Sridharan et al., 2008). Heilbronner and Hayden (2016) agree that the dACC incorporates all task-relevant information, but propose that it guides actions. The dACC thereby has the role of pre-premotor cortex being the superior of the motor pathway.

\subsection{Eliciting cognitive control}

While many circumstances activate the $\mathrm{CCN}$, two specific cognitive tasks were used in this thesis.

\subsubsection{Stroop task}

The Stroop effect is widely studied in cognitive science (MacLeod, 2015). The effect is named after John Ridley Stroop who first described the effect in English (Stroop, 1935). Reading a word is a habituated behavior while naming its ink color is not. Extensive practice automates reading, whereas naming objects or their properties remains laborious (Cattell, 1886). Therefore, the meaning of a color word is processed automatically, while naming the ink color takes conscious effort. Color words contain two color stimuli - the meaning of the word itself and the ink color. The stimuli match in the word Red (congruent: Word meaning = red ink color), but do not in Red (incongruent: Word meaning $\neq$ blue ink color). Klein (1964) showed that ignoring any word meaning is impossible, but the Stroop effect diminishes when words were associated only 
loosely with colors. For example, the word 'blood' is associated with the color red, but less so than the word red. The Stroop effect is the difference in response time between congruent and incongruent trials. The latter are of high-conflict and activate the CCN stronger than the congruent/low-conflict words (Hanslmayr et al., 2008).

The brain regions whose activity is most commonly associated with the Stroop task are dACC, DLPFC, and PPC (Banich et al., 2000; Kerns et al., 2004; Silton et al., 2010).

\subsubsection{Instrumental learning and Pavlovian biases}

Organisms learn during their lifetime to better navigate their surroundings and optimize their behaviors to achieve goals. Behaviors are either conductive to or detrimental to reaching goals. The outcome of a behavior usually serves as direct feedback about the appropriateness of the behavior. In instrumental learning a behavior's occurrence is changed by two types of feedback, reinforcement or punishment. Reinforcement increases the occurrence of a behavior and punishment decreases the occurrence.

Reinforcement is subdivided into positive and negative reinforcement. Positive hereby means that something is added and negative means that something is subtracted. Positive reinforcement thus consists of a reward post-behaviorally. Negative reinforcement on the other side can be either the removal of noxious stimulus after behavior (Escape) or the behavior itself could avoid the noxious stimulus (Active Avoidance). In both cases a noxious stimulus is removed, thus the behavior is associated with an improved outcome.

Similarly, punishment also consists of a positive and a negative component. Adding a noxious stimulus after behavior is a positive punishment, while removing a beneficial stimulus is a negative punishment. The net outcome is worse than if the behavior was not performed. The frequency of the behavior is thus expected to decline.

Instrumental learning therefore relies on action-outcome associations. It was termed operant conditioning originally by Skinner in the 1930s. Reinforcement/punishment and positive/negative are orthogonal. Two different strategies exist for reinforcing or punishing a behavior.

Predictable failures of instrumental learning stem from the influence of the evolutionary conserved Pavlovian motivational system (Cavanagh et al., 2013; Holland, 1979; Hershberger, 1986). This system tightly biases approach behavior to beneficial outcomes and conversely 
avoidance behavior to noxious outcomes. While this often facilitates instrumental learning, it conflicts sometimes with the higher-order, more flexible instrumental learning. If approaching is leading to noxious outcomes or avoidance is beneficial, the hard-wired custom to approach to gain and avoid to not lose is in conflict and interferes with the instrumentally learned actionoutcome associations. In animals these Pavlovian biases sometimes even prevent the instrumental learning. Chicken and rats were unable to avoid rewards or reward signals even if they could only obtain them through avoidance behavior (Hershberger, 1986; Holland, 1979). But also in humans Pavlovian biases influence behavior, which can conflict with instrumentally learned actions (Cavanagh et al., 2013).

\subsection{Neural oscillations}

The tasks described in the earlier subchapters require multiple, sometimes distant brain regions to commonly process information over varying time periods in order for the right behavior to be executed. The intercellular communication between neuron has for long been studied and is the basic unit of information transmission. However, this local phenomenon is insufficient to explain the integration of information between distant brain regions (Singer and Gray, 1995). Neural oscillations have emerged as a probable, evolutionary-conserved mechanism of longrange information transmission (Buzsáki et al., 2013).

Neural oscillations are rhythmic changes in neural activity in the brain. While these rhythms can appear in individual neurons, complete neural ensembles with many synchronized neurons produce macroscopic oscillations that traverse the brain and can be measured with electroencephalography (EEG; Jackson and Bolger, 2014). Classically, oscillatory activity falls into three distinct categories of scale: Single neurons (micro-scale), local groups of neurons (meso-scale) and activity of brain regions (macro-scale).

Neural oscillations are physically characterized by frequency, phase and amplitude. Macro-scale oscillations measured by EEG are classified into frequency bands that in recent years were correlated to specific brain functions. While delta $(1 \mathrm{~Hz}-4 \mathrm{~Hz})$ is associated with sleep, theta $(4 \mathrm{~Hz}$ - $8 \mathrm{~Hz})$, alpha $(8 \mathrm{~Hz}-13 \mathrm{~Hz})$, beta $(13 \mathrm{~Hz}-30 \mathrm{~Hz})$ and gamma $(30 \mathrm{~Hz}-140 \mathrm{~Hz})$ reflect the plethora of brain processes during wakefulness. Heuristically, the wider oscillatory communication spans, the lower the frequency. Thus, wide-scale coordination in sleep occurs in the lowest frequency band, while local groups of neurons rely on gamma range activity (Nyhus and Curran, 
2010). The precise underlying mechanisms of neural oscillations are still not understood, but it is known that inhibitory interneurons simultaneously change the membrane potentials of many cortical, excitatory pyramidal cells.

Macro-scale neural oscillations are often measured with EEG non-invasively (Buzsáki et al., 2012). It allows the measurement of electric activity close to the surface of the brain. In particular, the postsynaptic potentials of cortical pyramidal cells dominates the EEG signal (Voytek and Knight, 2015). The temporal resolution is sufficient to capture all relevant brain processes, but the spatial resolution is limited. As the electrical signal propagates through dura mater and pia, the skull and the skin, the measured voltage changes at the scalp are mixed from multiple sources. Thus, while an increasing number of EEG electrodes make measurements more finegrained, the estimation of sources of EEG activity is laborious. Nonetheless, between its easy, non-invasive application and high temporal resolution, EEG is an important electrophysiological readout method for neural oscillations.

Distant brain regions communicate when phases of their oscillations match (Fell and Axmacher, 2011; Bonnefond et al., 2017). Either the brain regions transmit in the same frequency or crossfrequency coupling (CFC) occurs (Canolty and Knight, 2010). CFC, often, describes that the phase of a frequency modulates the amplitude of the other. Additionally, information transmission between brain regions requires an appropriate lag (Fries, 2005). The information flows directionally from the leading to the lagging brain region (Fries, 2005).

Changes in macro-scale oscillations is associated with neurological disorders and altered states of consciousness. Pathologically, Parkinson's disease, for instance, is characterized by failures in long-range synchronization (Schnitzler and Gross, 2005). Abnormal synchrony and amplitude of high-frequency neural oscillations occur in schizophrenia (Uhlhaas and Singer, 2013). Transient alterations of wakefulness by drugs like psilocybin are marked by broadband cortical desynchronization (Muthukumaraswamy et al., 2013).

Theta range neural oscillations occur in DLPFC and dACC during Stroop task and instrumental learning requiring the control of Pavlovian instincts (Hanslmayr et al., 2008; Cavanagh et al., 2013). It is commonly known as frontal midline theta rhythm of which dACC is considered to be the source (Inanaga, 1998; Ishii et al., 1999). This frontal midline theta rhythm presumably is tightly linked to the EVC and therefore signals and/or implements control (Cavanagh and Frank, 2014). 


\subsection{Transcranial electrical stimulation}

Both physiological and pathological electrical activity of the brain can be modulated by applying electrical currents through the skull by low-intensity transcranial electrical stimulation (tES , Rossini et al. (2015); Polanía et al. (2018)). The impact of tES on physiological neuronal activity and plasticity is studied in basic cognitive research (for an introduction see Yavari et al. (2018)). At the same time, tES promises advances in the treatment of neurological and psychiatric disorders (Fertonani and Miniussi, 2017). However, that tES is easy-to-apply has been confounded with easy-to-use (Fertonani and Miniussi, 2017). This has led to a varied application without complete understanding of the mechanistic underpinnings.

During tES, voltage differences between electrodes that are mounted to the head induce current flow in the brain. This exogenously induced electric current does not induce action potentials but modulates the resting membrane potential, which in turn modulates the firing rate of neurons (Polanía et al., 2018). The current is strongest right under the electrodes, which are therefore often placed over the region of interest. The spatial resolution of tES is low, and while the time resolution is very high compared to brain processes, currents are usually applied over a long time period (Polanía et al., 2018).

Two types of basic tES exist: Transcranial direct current stimulation (tDCS) and transcranial alternating current stimulation (tACS) with its notable subtype transcranial random noise stimulation (tRNS). tDCS is the most commonly used form of tES (Nitsche and Paulus, 2000; Polanía et al., 2018). Here, direct currents flows constantly between one or more cathodes to one or more anodes. Cathodal stimulation is thought to decrease excitability in underlying neurons by shifting the membrane potential. Anodal stimulation acts inversely and increases the excitability. In tACS sinusoidally oscillating current flows between the electrodes (Antal et al., 2008). The application of a single frequency induces the neurons in the target region to synchronize to it (Antal and Paulus, 2013). The neurons' resting membrane potentials oscillate in the applied frequency, which directly affects their firing rates (Antal and Paulus, 2013; Herrmann et al., 2013). Consequentially, the oscillation frequency of tACS is transmitted to the oscillatory system in the brain, a process called entrainment (Helfrich et al., 2014). The entrainment also spreads from the target area to more distant ones (Schutter, 2014).

Transcranial random noise stimulation transmits white noise distribution of currents within a 
wide frequency spectrum (often $0.1 \mathrm{~Hz}-1000 \mathrm{~Hz}$ ). Blinding is easier with tRNS as the perception threshold in the skin is higher than compared to tDCS (Ambrus et al., 2010). The precise mechanism of action is not clarified completely (Antal and Herrmann, 2016). Both repetitive opening of sodium channels or stochastic resonance might contribute (Schoen and Fromherz, 2008; Stacey and Durand, 2000). For instance, increased cortical excitability and better cognitive performance have been induced after stimulation of intensities between $1 \mathrm{~mA}$ to $2 \mathrm{~mA}$ and durations between 10 min to 20 min (Terney et al., 2008; Pasqualotto, 2016).

The online (acute) effects of tES as described above are observed only during stimulation. They are ascribed to the change in membrane potentials induced by the current (Lefaucheur et al., 2017). After-effects, however, are observed even minutes or hours later and are hypothesized to reflect changed neuronal excitability that arises from altered synaptic connections (Antal and Herrmann, 2016).

Neural oscillations are ubiquitous in the brain and strongly correlated with specific cognitive processes. This implies that they are functionally important. However, due to the correlative nature of oscillations and cognitive processes, the neural oscillations also might be mere epiphenomena. Then they would be caused by cognitive processes, but not be causally involved in the function.

For example, every car emits exhaust fumes; the assumption that these fumes propel the car forward is false. In recent years, interest has grown in the question: Are neural oscillations by-products, like fumes, or more like the transmission, driving the car forward?

We know that different behaviors require different neural oscillation patterns to appear. Studying if and how manipulated neural oscillation patterns change behavior can elucidate if neural oscillations are causally and by which mechanism influencing brain processes. tACS is especially suited; its mechanism of changing the physiological oscillations is well-studied.

\subsubsection{Technical limitations of tES}

Like every other method, tES possesses technical limitations. Due to the distance of the electrodes from the target area and the high conductivity of biological tissue (excluding bone tissue), the spatial resolution is limited. The electric current flows through the path of least resistance, which means that often the skin and the cerebrospinal fluid conduct most of it. Nonetheless, the electric current also reaches non-targeted brain areas. Newer electrode placement strategies 
like a main electrode surrounded by multiple return electrodes allow more accurate targeting (Edwards et al., 2013; Villamar et al., 2013), although the resolution of transcranial magnetic stimulation is (currently) unattainable.

Finally, tES has historically been used to influence brain regions close to the surface of the brain. The electric field strength diminishes with increasing distance from the electrodes. Electric field strengh of $0.3 \mathrm{mV} / \mathrm{mm}$ are commonly assumed to be necessary for neural and behavioral effects, although the threshold might be higher (1 mV / mm; Vöröslakos et al. (2018)). So, inducing changes in brain physiology is less difficult in the periphery of the brain, but clinically relevant brain areas can be located more centrally (e.g. deep-brain stimulation targets the substantia nigra of the mesencephalon). One hub of the $\mathrm{CCN}$, the dACC, is not at the brain surface. Therefore, stimulating it constitutes progress in expanding the usability of tES van Driel et al. (2015); Onoda et al. (2017). This thesis partially shows that the dACC is a valid, reachable target for tES and a functionally important hub of the CCN.

\subsection{Objectives and structure of this dissertation}

The objectives of this thesis were to characterize the CCN further by stimulating the left DLPFC and the dACC during instrumental learning and during Stroop task.

- In Chapter 2, the left DLPFC was targeted as proof-of-concept that theta-range tACS influences the CCN (Lehr et al., 2019).

- In Capter 3, the dACC was targeted during Stroop task also in theta-range tACS.

- The stimulation of the dACC as a deeper brain area furthers the application spectrum of tES

- In Chapter 4, cross-frequency tACS, that nested gamma oscillations into theta oscillations, was targeted to the dACC,

- This allows to generalize the results of tES stimulation on CCN across differing cognitive requirements.

- Throughout all these chapters, the causal importance of the DLPFC and dACC during tasks requiring cognitive control was investigated. 
In Chapter 2, mid-theta range tACS over the DLPFC reduced the behavioral Stroop effect. In Chapter 3, mid-theta range tACS targeting the dACC reduced the behavioral Stroop effect, but no clear effects persisted after stimulation. In Chapter 4, cross-frequency tACS to the dACC worsened cognitive control during a Go/NoGo instrumental learning task.

The general discussion (Chapter 5) puts the results of this thesis into greater context and dissects scientific limitations of tES that have governed this thesis. 
2 MODULATION OF CONFLICT PROCESSING BY THETA RANGE TACS OVER THE DORSOLATERAL PREFRONTAL CORTEX

\section{Modulation of conflict processing by theta range tACS over the dorsolateral prefrontal cortex}

Authors: Albert Lehr ${ }^{1}$, Niklas Henneberg ${ }^{1}$, Tarana Nigam ${ }^{1}$, Walter Paulus ${ }^{1}$ and Andrea Antal ${ }^{1}$ Affiliation: ${ }^{1}$ Department of Clinical Neurophysiology, University Medical Center Göttingen, Göttingen 37073, Germany

Neural Plasticity, published

doi: https://doi.org/10.1155/2019/6747049

Author contributions:

Detailed author contribution of A. Lehr: conceptualization, study design, project administration, methodology, software (behavioral paradigm), supervised data collection, supervised NH \& TN, formal analysis, prepared all illustrations, data visualization, interpreted data, data curation and wrote original draft.

$\mathrm{NH}$ : data collection for second experiment

TN: data collection for first experiment, formal analysis

AA: project administration, supervised NH \& MS, contributed to writing original draft.

WP: resources and funding acquisition, contributed to writing original draft.

The citation style of the publication has been changed to match the citation style of the dissertation. Its bibliography is included in the general bibliography of the dissertation.

Copyright (C) 2019 Albert Lehr et al. This is an open access article distributed under the Creative Commons Attribution License, which permits unrestricted use, distribution, and reproduction in any medium, provided the original work is properly cited. 


\subsection{Abstract}

Behavioral response conflict arises in the color-word Stroop task and triggers the cognitive control network. Midfrontal theta-band oscillations correlate with adaptive control mechanisms during and after conflict resolution. In order to prove causality, in two experiments, we applied transcranial alternating current stimulation (tACS) at $6 \mathrm{~Hz}$ to the dorsolateral prefrontal cortex (DLPFC) during Stroop task performance. Sham stimulation served as a control in both experiments; 9.7 Hz tACS served as a nonharmonic alpha band control in the second experiment. We employed generalized linear mixed models for analysis of behavioral data. Accuracy remained unchanged by any type of active stimulation. Over both experiments, the Stroop effect (response time difference between congruent and incongruent trials) was reduced by $6 \mathrm{~Hz}$ stimulation as compared to sham, mainly in trials without prior conflict adaptation. Alpha tACS did not modify the Stroop effect. Theta tACS can both reduce the Stroop effect and modulate adaptive mechanisms of the cognitive control network, suggesting midfrontal theta oscillations as causally involved in cognitive control.

\subsection{Introduction}

In the face of conflicting information, human beings are capable of adjusting their executive control to resolve conflict and perform the appropriate behavior.

During this process, the cognitive control network first detects conflict, then selects and monitors behaviors for attaining a goal. Multiple brain regions jointly exercise inhibitory control when task demands are high to override stimulus-driven behavior. Generally, cognitive control is measured by performance in conflict tasks, like the Stroop task, in which conflicting task-irrelevant information has to be suppressed for responding correctly (Cole and Schneider, 2007; Stroop, 1935).

In the Stroop color-word task (SCWT), participants indicate the ink color of a color-word while not responding to its semantic meaning. Responses are faster when the semantic meaning and ink color match (congruent, low-conflict, e.g., "Blue" in blue ink) compared to a mismatch (incongruent, high-conflict, e.g., "Blue" in red ink). This response time difference is a function of the congruence and named after its discoverer Stroop (MacLeod, 2005).

Previous electroencephalography (EEG) and functional magnetic resonance imaging (fMRI) 
studies found that several brain regions are activated during the Stroop task, including the dorsal Anterior Cingulate Cortex (dACC), the dorsolateral prefrontal cortex (DLPFC), and the posterior parietal cortex (PPC) (Banich et al., 2000; Kerns et al., 2004; Silton et al., 2010). Neuroimaging studies suggest that the left DLPFC is active $(300 \mathrm{~ms}-440 \mathrm{~ms})$ before the dACC (520 ms$680 \mathrm{~ms}$ ), indicating the left DLPFC as the source of cognitive control implemented for Stroop task performance (Banich et al., 2000; Silton et al., 2010). Contrarily, the dACC has also been hypothesized to detect conflict at an earlier point in time (220-340 ms) and to engage the DLPFC that then implements cognitive control and resolves the conflict (Silton et al., 2010; Carter and van Veen, 2007). This apparent contradiction resolves as cognitive control is exerted strongly in trials following an incongruent trial.

Responses in incongruent trials, which are preceded by incongruent trials (iI), are faster than in incongruent trials, which are preceded by congruent trials (cI). Conversely, responses in cI are slower than in cC (Kerns et al., 2004). This congruency sequence effect (CSE) in trials preceded by incongruent trials is known as the Gratton effect (Gratton et al., 1992). A conflict in the previous trial recruits greater cognitive control that modulates response times in the subsequent trial. This behavioral adjustment is predicted by the conflict adaptation hypothesis (Carter et al., 1998; Botvinick et al., 2001, 2004). The dACC activity increases in conflict trials (Botvinick et al., 2004). It precedes behavioral adaptations promoted by increased DLPFC activity. Thus, this theory postulates that the interplay of conflict-detecting dACC and allocation of control by the DLPFC is responsible for adaptation of the congruency effect. As conflict trials activate the dACC, engagement of DLPFC reduces both the Stroop effect and the dACC activity in trials after a conflict (Kerns et al., 2004). If the DLPFC activity is high after engagement, it abolishes the Stroop effect independent of the dACC activation level (Silton et al., 2010). Only when the DLPFC activity is low (no earlier engagement by dACC) will the dACC activity correlate with the size of the Stroop effect and negatively with error rates (Silton et al., 2010). Therefore, it is conceivable that constant high activation of the DLPFC throughout the Stroop task leads to the abolishment of the Stroop effect. In this study, we aim to increase DLPFC activity exogenously to test this hypothesis.

Transcranial alternating current stimulation (tACS) allows us to causally infer function of oscillatory networks (Antal et al., 2008). Through the injection of alternating current into the cortex, membrane potentials of many neurons are rhythmically and simultaneously shifted (Ali et al., 
2013). This effectively entrains networks exogenously (Helfrich et al., 2014). By controlling the rhythmic brain activity, resulting changes in cognitive functions can be causally attributed to the brain oscillation.

In this study, we have chosen tACS with a frequency of $6 \mathrm{~Hz}$ based on previous electrophysiological results. Generally, these electrophysiological studies are in line with and corroborate the findings of neuroimaging studies in the Stroop task. The dACC has been shown to be the generator of mediofrontal negativity in the theta $(4-8 \mathrm{~Hz})$ range marked by a stronger negative potential around $450 \mathrm{~ms}$ in the incongruent condition (West and Alain, 1999; Liotti et al., 2000; West et al., 2005). This midfrontal theta-band (4-8 Hz) oscillatory activity supposedly reflects neural mechanisms of conflict detection (Cavanagh and Frank, 2014).

Furthermore, dACC and left DLPFC couple in the theta phase between conflict detection and resolution (Hanslmayr et al., 2008). In this phase, dACC activity predicts DLPFC activity, establishing dACC as the driving brain region (Oehrn et al., 2014). This intra-areal theta connectivity is prolonged in incongruent compared with congruent trials (Hanslmayr et al., 2008). Similar to the Gratton effect for response times, oscillatory power in narrow-band theta $(6 \mathrm{~Hz}-7 \mathrm{~Hz})$ in the left-frontal region is significantly higher in $\mathrm{iC}$ trials compared to $\mathrm{cC}$ trials, while it is slightly lower in iI trials compared to cI trials (Tang et al., 2013). Additionally, non-phase-locked theta power correlates with response times (Cohen and Donner, 2013). Thus, the evidence suggests that theta power in the left-frontal region and response times are both influenced by conflict in preceding trials. Natural increase in frontocentral theta power and phase-coupling between dACC and left DLPFC in conflict mediate the increased conflict adaption in the next trial. In the Simon task, the congruency effect was reduced during theta-range tACS directed medially towards the dACC because response times slowed in congruent trials (van Driel et al., 2015). However, in the color-word Stroop task, the evidence for the importance of dACC and DLPFC interaction for the successful resolution of conflict remains correlational. By stimulating the DLPFC, we aim to illuminate the role of the DLPFC in the cognitive control network during the performance of the Stroop task.

Similarly to our approach, a previous work has also targeted the left DLPFC with a theta-range tACS during decision-making requiring cognitive control (Sela et al., 2012). Stimulation increased riskier decision, which confirms the DLFPC as a key region for adaptation of decision strategies. Likewise, theta-range tACS to the left DLPFC increased performance in the 
easy items of a problem solving test by changing attentional components (Pahor and Jaušovec, 2014). The stimulation did however not improve performance in a visual-spatial reasoning task. These results indicate DLPFC specifically as a promising target for low-frequency tACS during cognition, while numerous studies have shown transcranial electrical stimulation to modulate cognitive processes in general (Kuo and Nitsche, 2012).

To investigate the efficacy of tACS on conflict processing, we have used the drift diffusion model for conflict tasks (DMC). The DMC is a newly developed extension of the classical drift diffusion model (DDM) (Ratcliff, 1978; Ulrich et al., 2015).

Generally, cognitive processing in conflict tasks is studied by behavioral measures like response time and accuracy, which are influenced by a trade-off between speed and accuracy of response. Cognitive models allow decomposing the response time and accuracy into several parameters underlying the decision process. The DDM models the cognitive processes underlying twoalternative forced choice tasks by assuming that participants start to accumulate for either alternative over the time of the trial. The accumulation of evidence begins at the start of the trial, and as soon as it reaches a certain threshold for one alternative, a decision is being made. Due to noisy sensory input, the accumulation is a stochastic process which occasionally results in error trials. Aside from the decision process, the time needed for nondecisional processes is also accounted for.

In DMC, evidence accumulation is the sum of a controlled process (naming of color) and another, automatic process (recognition of semantic meaning). These processes are summed, either leading to (slower) faster responses in (in)congruent trials. The distribution in time of the automatic process is a gamma density function, peaking early during the trial and decaying afterwards. Therefore, the DMC is well suited as it accounts for both the RT distributions and accuracies of conflict tasks as Stroop, Simon, or Eriksen flanker task (Simon and Rudell, 1967; Eriksen and Eriksen, 1974).

We aimed to externally modulate theta power in the left DLPFC and to thereby causally change the function of the cognitive control network. We employed tACS in the theta range $(6 \mathrm{~Hz})$ with a high-definition (HD) electrode montage over the left DLPFC in two experiments, in order to entrain the cortical control network (Edwards et al., 2013; Villamar et al., 2013). While both experiment stimulations were compared to sham, the second experiment additionally used tACS in the alpha range $(9.7 \mathrm{~Hz})$ as a control. This serves as an active control for the possible 
frequency-unspecific effects of stimulation.

As mentioned above, we employed GLMM and the newly developed DMC to analyse the effects of tACS on response times and accuracy and also the interaction with the congruency effect (Ulrich et al., 2015). We hypothesized that the cortical control network can be exogenously entrained (via the left DLFPC) by theta tACS. This would result in increased theta power during and after conflict resolution. With longer phase-coupling between the dACC and the left DLPFC, all trials would show activation patterns similar to those in the incongruent trial. This would induce higher cognitive control for the next trial, comparable to the $\mathrm{iC}$ or iI conditions of the Gratton effect. Therefore, we predicted a reduced Stroop effect in the active condition compared to the controls. We expected trials which are preceded by a congruent trial to be more strongly affected by stimulation (reduced Stroop effect) as normally they show no conflict adaptation mediated by theta phase-coupling. Consequently, in DMC, the influence of the automatic process on the decision-making should be reduced.

\subsection{Methods}

\subsubsection{Participants}

The participants consisted of 22 healthy, right-handed, and native German-speaking adult volunteers, who have normal or corrected-to-normal vision and gave their written informed consent to join the study. They were measured in two experimental groups. The first group consisted of 10 participants ( 8 females, mean age: $244 \pm 38$ years); the second group consisted of 12 participants (8 females, mean age: $25 \pm 37$ years). None of the participants reported neurological or psychiatric disorders and drug-dependency or were taking medication acting on the central nervous system prior to or during the experimental sessions. They were informed about the exclusion criteria and possible adverse effects of tACS. The Ethics Committee of the University Medical Center of Göttingen, Germany, approved the study, which was conducted according to the regulations of the 1964 Declaration of Helsinki.

\subsubsection{Experimental protocol}

The experiments were double-blinded, placebo-controlled, and executed in a within-subject design. Experiment $1(\mathrm{n}=10)$ consisted of an active $6 \mathrm{~Hz}$ tACS and a sham stimulation session. 
Experiment $2(\mathrm{n}=12)$ had an additional active control condition (alpha tACS). Subjects participated in all sessions of a given experiment. The condition order was counterbalanced across participants to minimize learning effects. Between experimental sessions, a duration of at least 48 hours was maintained to diminish possible carry-over effects of stimulation. Before and after each session, participants reported their level of arousal and indicated their subjective experience of the stimulation after the session. The dependent variables in this study were accuracy and response times (RTs). Additionally, the arousal and sleep quality were also reported.

\subsubsection{Task}

Participants performed a Stroop color-word task (SCWT) (Stroop, 1935), which was designed using the PsychoPy toolbox (Peirce, 2007). In the SCWT, the participants have to indicate the color of the font. The stimuli were four German capitalized color-words (Green, Red, Yellow, and Blue) presented with matching or different font colors. The task was designed as a twoalternative forced choice task, meaning that two colors (Green and Red) mapped onto the same one of the two response buttons that the participant had to press manually. Responses were collected by a dedicated response pad (RB-740; Cedrus Corporation, San Pedro, USA) with a time resolution of $2 \mathrm{~ms}$ to $3 \mathrm{~ms}$ according to the manufacturer. The congruent condition consisted of the matching color-word and font color (e.g., RED written in red). In the incongruent condition, the color-word and the font color were different but also mapped onto different buttons (e.g., RED in yellow). The CIE Lightness Chroma hue device-independent colorimetric space (Commission Internationale de l'éclairage, 1976) was applied. Red (hue = 30), Blue (hue = 280), Green (hue $=140)$, and Yellow (hue $=100)$ had the same lightness $(\mathrm{L}=51)$ and chroma level $(\mathrm{CL}=55 \%)$. The gray fixation cross had the same lightness.

Each session started with a minimum of 50 practice trials (termination rule: 18 of the last 20 trials correct), and the following main phase consisted of 300 congruent and incongruent trials in a randomized order. The length of a trial was $1.5 \mathrm{~s}$; the mean interstimulus interval lasted $0.5 \mathrm{~s}$ (Chi-squared distribution, range $0.3 \mathrm{~s}-0.7 \mathrm{~s}$ ) during which a gray fixation cross (hue) was shown. The participants were instructed to respond as quickly and accurately as possible. The SCWT lasted for 20 minutes (Figure 4). 
2 MODULATION OF CONFLICT PROCESSING BY THETA RANGE TACS OVER THE DORSOLATERAL PREFRONTAL CORTEX

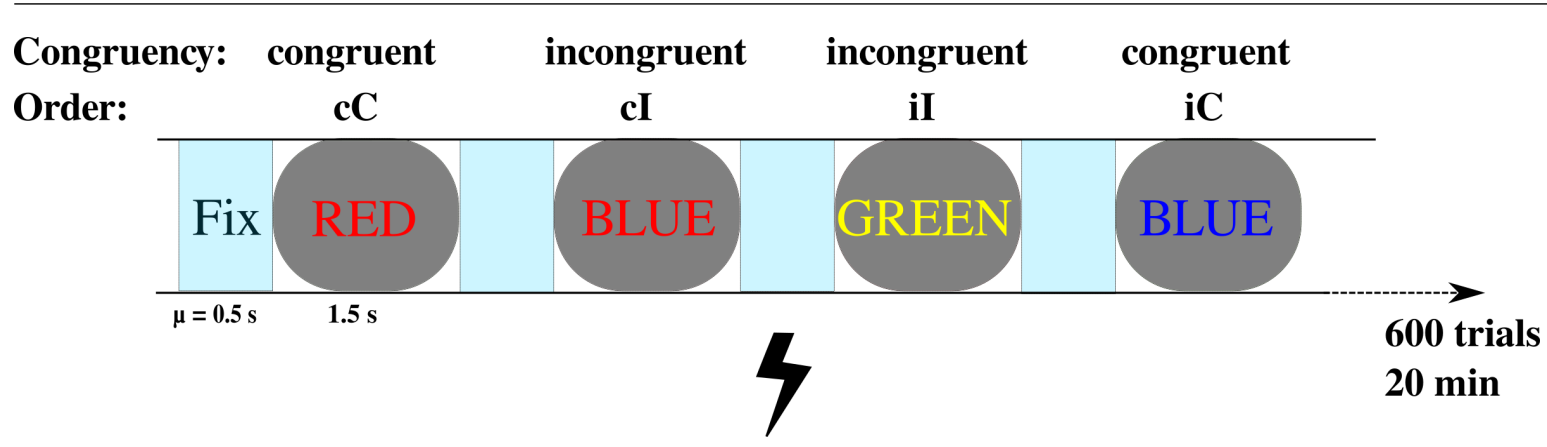

Figure 4: The color-word Stroop task. After practice trials, the participants performed 600 trials within one session while being stimulated by tACS. They responded as quickly and accurately as possible during the $1.5 \mathrm{~s}$ of a single trial. Congruent and incongruent trials appeared equally often and were subcategorized depending on the preceding trial.

\subsubsection{Transcranial alternating current stimulation}

Stimulation was delivered by a CE-certified neuroConn multichannel stimulator (neuroConn GmbH, Ilmenau, Germany) throughout the main experimental phase (Antal et al., 2008). The high-definition (HD) montage centered over AF3 according to the international 10-10 EEG system with four return electrodes. The return electrodes were positioned over F5, F2, Fp2, and AF7 as in earlier studies targeting the DLPFC (de Lara et al., 2017). In previous studies, this electrode positioning was used to modulate the activity of the DLPFC. Following the recommendation of previously published modelling studies, the orientation of the plugs and cables was kept constant (facing away perpendicular to the medial line) (Saturnino et al., 2015). Round rubber electrodes of $1 \mathrm{~cm}$ radius were fixed on the scalp of participants with the conductive Ten20 paste. This placement leads to left hemispheric frontal stimulation with peak field intensities of $0.3 \mathrm{~V} / \mathrm{m}$ (Figure 5) according to simulations with the SimNIBS standardized head model (Thielscher et al., 2015).

Sinusoidal tACS of $1 \mathrm{~mA}$ (peak-to-baseline) intensity and $6 \mathrm{~Hz}$ frequency was applied throughout the 20 min duration of the WCST in the active stimulation condition (including $10 \mathrm{~s}$ ramp-up and ramp-down periods). Similarly, $9.7 \mathrm{~Hz}$ was used as an active control stimulation in the alpha range in the second experiment. Sham stimulation was limited to $30 \mathrm{~s}$ (including $10 \mathrm{~s}$ ramp-up and ramp-down periods) during the beginning and the end of the SCWT in order to blind the participants while not influencing task performance. The impedances were kept below $15 \mathrm{k} \Omega$. The current density at the main electrode was $0.159 \mathrm{~mA} / \mathrm{cm}^{2}$. 
A

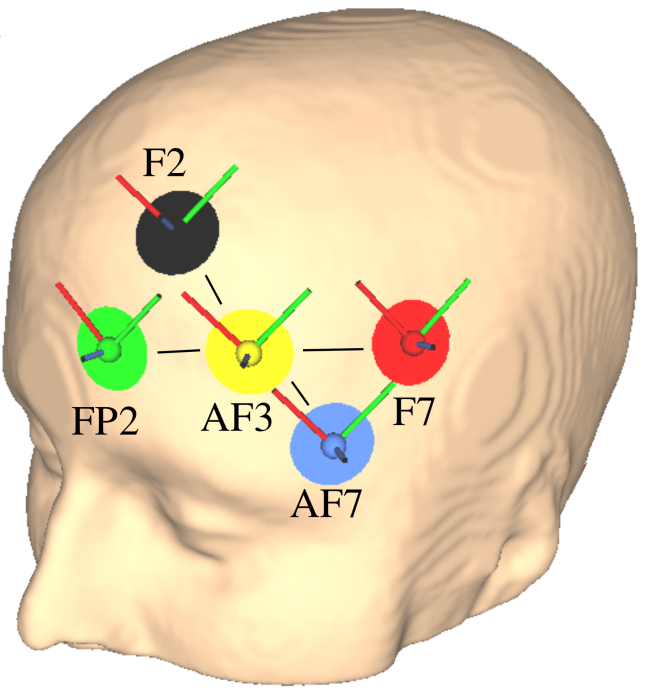

B

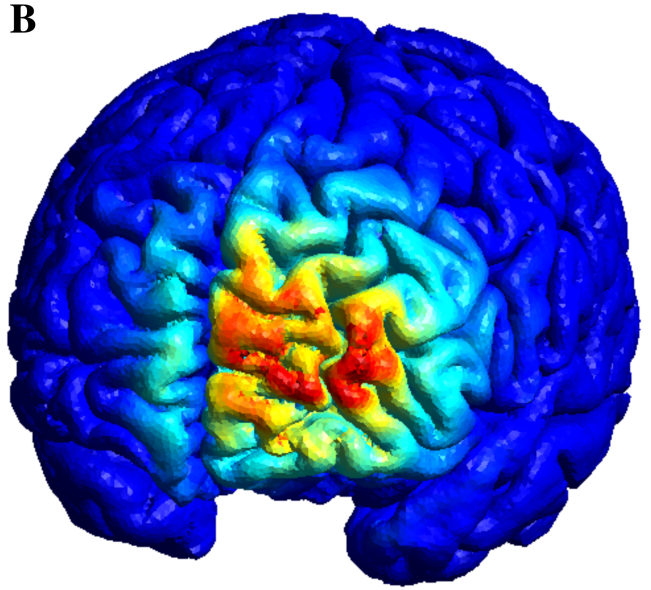

0
$\mathrm{mV} / \mathrm{mm}$
0.35

Figure 5: The HD tACS montage for stimulation of the left dorsolateral prefrontal cortex and the modelled electric field strength. (a) The central electrode of the HD montage is centered over AF3. Two pairs of return electrodes form equilateral triangles of $6 \mathrm{~cm}$ side length with the central electrode. The distance between both pairs is 10 $\mathrm{cm}$. The return electrodes are located over F5, Fp2, F2, and AF7. (b) The electric field strength is maximal (0.35 $\mathrm{mV} / \mathrm{mm}$ ) over the left prefrontal cortex including the DLPFC. The graphics and electric field strength modelling are derived from SimNIBS 2.0.1.

\subsubsection{Analysis}

The DMC fitting and the organization of behavioral datasets were done in Python. All statistical testing were conducted in R (R Core Team, 2018).

2.3.5.1 Generalized linear mixed models Generalized linear mixed models (GLMMs) are increasingly utilized to analyse complex research designs (Breslow and Clayton, 1993; Bolker et al., 2009). They are mainly used for correlated data, e.g., data in which many data points per individual participant exist (McCulloch, 2003). This hierarchical structure is analysed without using mean data averaged across the participants' responses. Response time distributions are normally right-skewed, but GLMM does not assume data to be normally distributed (Lo and Andrews, 2015). Overall, GLMM allows data to be analysed without reducing it first to mean values (Lo and Andrews, 2015).

Parsimonious GLMMs were run on nontransformed RTs of correctly answered trials using an identity-linked Inverse Gaussian distribution as recommended by Lo and Andrews (2015). Simi- 
larly, for error rates, the GLMM was run including incorrectly answered trials using an identitylinked binomial distribution. We fitted with the packages RePsychLing 0.0.4 (Baayen et al., 2015) and lme4 1.1-15 (Bates et al., 2014) following recommendations for nongeneralized models (Bates et al., 2015). Maximum likelihood was used to fit the GLMM.

The random effects in the final parsimonious model included intercepts for participants and word-color, with slopes of current trial congruency for word-color and within-participant slopes of current trial congruency and stimulation. The random effects account for variance in the data which arises as, for instance, every participant balances the speed-accuracy trade-off differently, which leads to individual response time and accuracy distributions. The categorical two-level fixed effects stimulation (sham, $6 \mathrm{~Hz}$ ), congruencies of current and preceding trials (both: congruent, incongruent), was sum-coded numerically for the first experiment. In the second experiment, the stimulation (sham, $6 \mathrm{~Hz}, 9.7 \mathrm{~Hz}$ ) was also sum-coded numerically, allowing the effect of the active stimulations to be individually compared to sham. Additionally, we could analyse the interaction of the stimulation with the current trial congruency (Stroop effect) and with the current and preceding trial congruencies (Gratton effect). These factorial predictors were contrast-coded to extract their main effects and their interactions on the grand means of reaction time and accuracy. We report the Z values and p values of the effects via Welch-Satterthwaite's approximation method (Kuznetsova et al., 2017). All data points are plotted with $95 \%$ prediction interval, which marks the range within which the data points would be with a probability of $95 \%$ upon resampling.

2.3.5.2 Fitting drift diffusion models for conflict tasks DMC assumes that the total response time is the sum of the duration of the decision process (D) and the residual time (R), which includes the sensory processing of stimulus and response execution (Ulrich et al., 2015). Additionally, it assumes that the congruency effect occurs only in the decision process. DMC decomposes the D underlying a two-alternative forced choice into several parameters by accounting for the RTs and accuracy of both congruent and incongruent trials. The boundary (a) is the threshold which has to be crossed by the evidence accumulation to elicit a decision. The nondecision (Ter) and the variability of the nondecision time $\left(\mathrm{s}_{r}\right)$ characterize R. A controlled process operates on task-relevant information and an automatic process on task-irrelevant information. The controlled process has a constant drift rate $\left(\mu_{c}\right)$, whereas the drift rate of the 
automatic process is changing over time best described by a gamma density function. It decays over time after an early maximum. The amplitude $(\zeta)$, shape parameter $(\alpha)$, and scaling parameter $(\tau)$ underlie the gamma function (Ulrich et al., 2015).

Model fitting was done on individual participants per session (and individual "original" datasets in the recovery study) as described in Ulrich et al. (2015) following these steps:

1. Plausible starting values from the pilot study were drawn for all parameters from a uniform distribution.

2. Minimization of $\mathrm{G}^{2}$ statistic as a goodness of fit of parameters to the RT distribution and accuracy was done by the Nelder-Mead simplex method (Nelder and Mead, 1965). The maximum number of iterations was 250 , each with a sample size of 50,000 observations per congruency condition. The integration constant (delta $\mathrm{t}=1 \mathrm{~ms}$ ) and diffusion coefficient (sigma $=4)$ were as in Ulrich et al. (2015).

3. The first two steps were repeated 30 times. Computations were done in parallel with the Göttingen Campus High-Performance Computing Center as each repetition had a run time of around $30 \mathrm{~h}$.

We further analysed the parameters which best fit the data as indicated by the $\mathrm{G}^{2}$ statistic. Parameters were statistically compared to infer which parameters had been influenced by the stimulation using permutation tests. The above-mentioned DMC parameters were the dependent variables with the stimulation condition being the independent variable. However, due to poor recovery, the shape and the time characteristic of the automatic process gamma function were excluded from this analysis (see supplementary Figure 7). Permutation tests are nonparametric tests. In the first experiment with its two stimulation conditions, approximative Monte Carlo Fisher-Pitman permutation tests were run for each analysed DMC parameter. As the second experiment included three stimulation conditions, we performed approximative multivariate Kruskal-Wallis tests. In both tests, 10,000 iterations were used (Hothorn et al., 2006, 2008). We adopted the hypothesis testing threshold according to the Bonferroni-Holm method for multiple testing.

2.3.5.3 Arousal and sleep Arousal levels in the Stroop task correlate with better performance in congruent trials and worse performance in incongruent trials (Pallak et al., 1975). For 
the control, participants self-reported their arousal level before and after performing the Stroop task on a scale from 1 (very tired) to 10 (totally awake). Sleep deprivation increases response times in the Stroop task but leaves interference and accuracy unchanged (Cain et al., 2011). Participants self-reported quality from 1 (miserable) to 5 (excellent) and duration (in hours) of their previous night's sleep. All indicators of each session were analysed across stimulation conditions using the two-sided nonparametric paired sample Wilcoxon signed rank test in Experiment 1 and the two-sided nonparametric paired sample Kruskal-Wallis test in Experiment 2.

\subsection{Results}

Table 1: Descriptive statistics of both experiments. For both experiments, the difference in behavior between congruent and incongruent trials is broken down per stimulation condition. Mean values are reported with their respective standard deviation.

\begin{tabular}{cll}
\hline & Accuracy $(\%)$ & Response times $(\mathrm{ms})$ \\
\hline Experiment 1 & $94.9 \pm 2.3$ & $624.3 \pm 54$ \\
Sham & & \\
Congruent & $95.9 \pm 2.8$ & $604.2 \pm 50.6$ \\
Incongruent & $94.6 \pm 2.4$ & $652.3 \pm 59.1$ \\
6 Hz tACS & & \\
Congruent & $95.3 \pm 2.9$ & $602.2 \pm 59.1$ \\
Incongruent & $93.7 \pm 2.7$ & $638.8 \pm 61.9$ \\
& & \\
Experiment 2 & $97.6 \pm 2.1$ & $578.1 \pm 57$ \\
Sham & & \\
Congruent & $98.2 \pm 1.7$ & $583.4 \pm 68.6$ \\
Incongruent & $97.8 \pm 1.5$ & $604.5 \pm 84.9$ \\
6 Hz tACS & & \\
Congruent & $97.6 \pm 2.5$ & $554.9 \pm 60.5$ \\
Incongruent & $96.9 \pm 3.4$ & $583.4 \pm 68.6$ \\
9.7 Hz tACS & & \\
Congruent & $98.1 \pm 1.9$ & $560.3 \pm 49.2$ \\
Incongruent & $97.0 \pm 2.3$ & $595.5 \pm 69$ \\
\hline
\end{tabular}

\subsubsection{First experiment}

Overall accuracy was $94.9 \%$ (SD $2.3 \%$ ), and mean RTs were $624.3 \mathrm{~ms}$ (SD $54 \mathrm{~ms}$ ). Within sham stimulation, accuracy was lower and mean RTs prolonged for incongruent trials $(94.6 \%$, 
SD $2.4 \%$; $652.3 \mathrm{~ms}$, SD $59.1 \mathrm{~ms})$ compared to congruent trials (95.9\%, SD $2.8 \%$; $604.2 \mathrm{~ms}$, SD $50.6 \mathrm{~ms})$. Equally, in the active stimulation condition, incongruent trials $(93.7 \%, 2.7 \%$; $638.8 \mathrm{~ms}, 61.9 \mathrm{~ms})$ were more erroneous and slower than congruent ones $(95.3 \%$, SD $2.9 \%$; $602.2 \mathrm{~ms}, 59.1 \mathrm{~ms}$ ) (see Table 1).

To assess the effect of the stimulation condition, we were interested in the main effect of the stimulation, its interaction with the congruency of the current trial and its effect on the Gratton effect (i.e., the interaction between congruency of the current and the previous trials). Additionally, we expected an interaction between congruency of the current trial and the stimulation conditions when the preceding trial was either congruent or incongruent. Two generalized linear mixed models were conducted: one for error rates including all trials and the other for the nontransformed response times excluding all error and posterror trials (10.4\% of all trials; see Table 2).

For accuracy, significant main effects exist for the congruency (congruent, incongruent) of the current trial $(\mathrm{CCT} ; \mathrm{Z}=2.801, \mathrm{p}<0.01)$ but not for stimulation $(\mathrm{Z}=1.875, \mathrm{p}=0.06)$ or the congruency of the preceding trial $(\mathrm{CPT} ; \mathrm{Z}=1.491, \mathrm{p}=0.13)$. Overall, participants were less accurate during incongruent trials $(\mathrm{M}=93.8 \%, \mathrm{SE}=0.7 \%)$ than during congruent trials $(\mathrm{M}=$ $95.3 \%, \mathrm{SE}=1.0 \%, \mathrm{p}<0.001)$. No effects were found for any higher-order interactions, including the interaction factors CCT $\mathrm{x}$ stimulation $(\mathrm{Z}=0.139, \mathrm{p}=0.88)$ or CCT $\mathrm{x}$ CPT $\mathrm{x}$ stimulation $(\mathrm{Z}=0.87, \mathrm{p}=0.38)$. The accuracy is only influenced by the CCT but not by stimulation or CPT. The analysis of the response times revealed significant main effects for CCT $(\mathrm{Z}=4.37, \mathrm{p}<$ $0.001)$ but neither for CPT $(Z=1.06, p=0.28)$ nor for stimulation $(Z=0.49, p=0.61)$. For $\mathrm{CCT}$, the response times were faster for congruent trials $(\mathrm{M}=600 \mathrm{~ms}, \mathrm{SE}=16.1 \mathrm{~ms})$ compared to incongruent $(\mathrm{M}=639.9 \mathrm{~ms}, \mathrm{SE}=17.6 \mathrm{~ms})$ trials (Stroop effect). The significant interaction CCT x CPT $(Z=3.026, p<0.01)$ constitutes the Gratton effect, in which the size of the Stroop effect depends on whether the CCT is preceded by a congruent $(\mathrm{M}=47.9 \mathrm{~ms}, \mathrm{SE}=7.1 \mathrm{~ms})$ or an incongruent $(\mathrm{M}=30.9 \mathrm{~ms}, \mathrm{SE}=4.1 \mathrm{~ms})$ trial. The interaction CCT $\mathrm{x}$ stimulation showed a trend $(Z=1.847, p=0.06)$ towards reduced Stroop effect under stimulation $(M=33.8 \mathrm{~ms}, \mathrm{SE}=$ $3.7 \mathrm{~ms})$ compared to sham $(\mathrm{M}=46.2 \mathrm{~ms}, \mathrm{SE}=7.1 \mathrm{~ms}$; see Figure 6). The triple interaction CCT $\mathrm{x}$ stimulation $\mathrm{x}$ CPT narrowly missed the significance criterion $(\mathrm{Z}=1.828, \mathrm{p}=0.06)$. Further exploration by dividing the dataset according to the congruency of the previous trial revealed a significant interaction CCT $\mathrm{x}$ stimulation for trials preceded by a congruent trial $(\mathrm{Z}=2.87, \mathrm{p}<$ 
$0.01)$ but no interaction if preceded by an incongruent one $(\mathrm{Z}=0.01, \mathrm{p}=0.98$; see Figure 6$)$. Thus, in trials preceded by congruent trials, the stimulation reduces the Stroop effect $(\mathrm{M}=35.8$ $\mathrm{ms}, \mathrm{SE}=6.1 \mathrm{~ms})$ compared to sham $(\mathrm{M}=60.2 \mathrm{~ms}, \mathrm{SE}=11.1 \mathrm{~ms})$.

\subsubsection{Second experiment}

Overall accuracy was 97.6\% (SD $2.1 \%$ ), and mean RTs were $578.1 \mathrm{~ms}$ (SD $57 \mathrm{~ms}$ ).

In sham stimulation, accuracy was lower and mean RTs prolonged for incongruent trials (97.8 $\%$, SD $1.5 \% ; 604.5 \mathrm{~ms}, \mathrm{SD} 84.9 \mathrm{~ms})$ compared to congruent trials $(98.2 \%$, SD $1.7 \% ; 569.5$ ms, SD $68.1 \mathrm{~ms})$. Equally, in the $6 \mathrm{~Hz}$ condition, incongruent values are $96.9 \%$, SD $3.4 \%$; $583.4 \mathrm{~ms}$, SD $68.6 \mathrm{~ms}$ and congruent values are $97.6 \%$, SD $2.5 \% ; 554.9 \mathrm{~ms}$, SD $60.5 \mathrm{~ms}$, and in the active control condition, incongruent values are $97.0 \%$, SD $2.3 \% ; 595.9 \mathrm{~ms}$, SD $69.0 \mathrm{~ms}$ and congruent values are $98.1 \%, \mathrm{SD} 1.9 \% ; 560.3 \mathrm{~ms}, \mathrm{SD} 49.2 \mathrm{~ms}$ (see Table 1).

To assess the effect of the stimulation condition, we were interested in the main effects of the two active conditions (stimulation: $6 \mathrm{~Hz}$; control: $9.7 \mathrm{~Hz}$ ). The two interactions were individually compared to sham stimulation. We further investigated their interaction with the congruency of the current trial and their effect on the Gratton effect (i.e., the interaction between congruency of the current and the previous trials). Additionally, we expected a change in their interaction between congruency of the current trial and the stimulation conditions when the preceding trial was either congruent or incongruent. Two generalized linear mixed models were conducted: one for error rates including all trials and the other for the nontransformed response times excluding all error and posterror trials (4.7\% of all trials; see Table 3$)$.

For accuracy, significant main effects existed for CCT $(Z=2.952, p<0.01)$ but not for CPT $(Z$ $=0.441, \mathrm{p}=0.65)$. Neither the stimulation $(\mathrm{Z}=0.579, \mathrm{p}=0.56)$ nor the control $(\mathrm{Z}=0.43, \mathrm{p}=$ 0.66) was significantly different from sham. Overall, participants committed more errors during incongruent trials $(\mathrm{M}=97.1 \%, \mathrm{SE}=0.6 \%)$ than during congruent trials $(\mathrm{M}=98.0 \%, \mathrm{SE}=0.5$ $\%)$. For stimulation compared to sham, no effects were found for any higher-order interactions, including the interaction factors CCT $x$ stimulation $(\mathrm{Z}=0.380, \mathrm{p}=0.703)$ and CCT $\mathrm{x}$ CPT $\mathrm{x}$ stimulation $(Z=0.876, p=0.38)$. For the control compared to sham, no effects were found for any higher-order interactions, including the interaction factors CCT $x$ stimulation $(Z=1.305, p$ 
Table 2: Statistical analysis of the first experiment. The results of the GLMMs are shown for both accuracy and response time data of the first experiment. Additionally, the response times were divided according to the congruency of the previous trial in additional model runs. For every factor, the mean values and standard errors of each factor level are reported. The results of the statistical testing of the difference between these mean values are also reported as $\mathrm{Z}$ and $\mathrm{p}$ values.

\begin{tabular}{|c|c|c|c|}
\hline & Estimate (mean $\pm \mathrm{SE})$ & | Z value | & $\mathrm{p}$ value \\
\hline \multicolumn{4}{|l|}{ Accuracy (\%) } \\
\hline CCT & $\Delta 1.5$ & 2.801 & $<0.01$ \\
\hline Congruent & $95.3 \pm 1.0$ & & \\
\hline Incongruent & $93.8 \pm 0.7$ & & \\
\hline CPT & $\Delta 0.6$ & 1.491 & 0.13 \\
\hline Congruent & $93.4 \pm 1.2$ & & \\
\hline Incongruent & $94.0 \pm 1.0$ & & \\
\hline Stimulation & $\Delta 0.9$ & 1.875 & 0.06 \\
\hline Sham & $94.2 \pm 1.1$ & & \\
\hline $6 \mathrm{~Hz}$ & $93.3 \pm 1.1$ & & \\
\hline \multicolumn{4}{|l|}{ Response times (ms) } \\
\hline CCT (Stroop effect) & $\Delta 39.9$ & 4.37 & $<0.001$ \\
\hline Congruent & $600 \pm 16.1$ & & \\
\hline Incongruent & $639.9 \pm 17.6$ & & \\
\hline CPT & $\Delta 3.2$ & 1.06 & 0.28 \\
\hline Congruent & $624.3 \pm 22.9$ & & \\
\hline Incongruent & $627.5 \pm 22.9$ & & \\
\hline Stimulation & $\Delta 6$ & 0.49 & 0.61 \\
\hline Sham & $628.9 \pm 22.7$ & & \\
\hline $6 \mathrm{~Hz}$ & $622.9 \pm 24.4$ & & \\
\hline CCT $\mathrm{x}$ stimulation & $\Delta 12.4$ & 1.847 & 0.06 \\
\hline Stroop effect (Sham) & $46.2 \pm 7.1$ & & \\
\hline Stroop effect $(6 \mathrm{~Hz})$ & $33.8 \pm 3.7$ & & \\
\hline CCT x CPT & $\Delta 17$ & 3.026 & $<0.01$ \\
\hline Stroop effect (CPT: congruent) & $47.9 \pm 7.1$ & & \\
\hline Stroop effect (CPT: incongruent) & $30.9 \pm 4.1$ & & \\
\hline CCT x CPT x stimulation & $\Delta 23.1$ & 1.828 & 0.06 \\
\hline \multicolumn{4}{|c|}{ Response times (ms) - data divided according to congruency of previous trial (CPT) } \\
\hline \multicolumn{4}{|l|}{$\mathrm{CPT}=$ congruent } \\
\hline CCT $\mathrm{x}$ stimulation & $\Delta 24.4$ & 2.87 & $<0.01$ \\
\hline Stroop effect (Sham) & $60.2 \pm 11.1$ & & \\
\hline Stroop effect $(6 \mathrm{~Hz})$ & $35.8 \pm 6.1$ & & \\
\hline \multicolumn{4}{|l|}{$\mathrm{CPT}=$ incongruent } \\
\hline CCT $\mathrm{x}$ stimulation & $\Delta 1.3$ & 0.01 & 0.98 \\
\hline Stroop effect (Sham) & $30.9 \pm 5.9$ & & \\
\hline Stroop effect $(6 \mathrm{~Hz})$ & $32.2 \pm 6.3$ & & \\
\hline
\end{tabular}

SE: Standard error. 
$=0.191)$ and CCT x CPT x stimulation $(\mathrm{Z}=1.572, \mathrm{p}=0.11)$. As in the first experiment, the accuracy is only influenced by the CCT but not by stimulation or CPT.

The analysis of the response times revealed significant main effects for CCT $(\mathrm{Z}=3.12, \mathrm{p}=$ 0.001) and for CPT $(Z=2.28, p=0.02)$. Compared to sham, neither the stimulation $(Z=0.78$, $\mathrm{p}=0.43)$ nor the control $(\mathrm{Z}=0.06, \mathrm{p}=0.95)$ had an effect on the response times. For CCT, the response times were faster for congruent $(\mathrm{M}=561.1 \mathrm{~ms}, \mathrm{SE}=15.0 \mathrm{~ms})$ compared to incongruent $(\mathrm{M}=592.4 \mathrm{~ms}, \mathrm{SE}=18.4 \mathrm{~ms})$ trials (Stroop effect). For CPT, the response times were faster when trials were preceded by congruent $(\mathrm{M}=5731 \mathrm{~ms}, \mathrm{SE}=165 \mathrm{~ms})$ compared to incongruent $(\mathrm{M}=580.3 \mathrm{~ms}, \mathrm{SE}=16.6 \mathrm{~ms})$ trials. The significant interaction CCT $\times$ CPT $(\mathrm{Z}=3.48, \mathrm{p}<$ 0.001) constitutes the Gratton effect, in which the size of the Stroop effect depends on whether the CCT is preceded by a congruent (Stroop effect: $\mathrm{M}=36.7 \mathrm{~ms}, \mathrm{SE}=7.6 \mathrm{~ms}$ ) or an incongruent (Stroop effect: $\mathrm{M}=24.9 \mathrm{~ms}, \mathrm{SE}=5.0 \mathrm{~ms}$ ) trial. The active stimulation significantly interacted with CCT $(Z=2.11, p=0.03)$ but not with the interaction CCT x CPT $(Z=0.35, p=0.72$; see Figure 6).

The size of the Stroop effect depends on whether participants were stimulated with $6 \mathrm{~Hz}$ tACS (Stroop effect: $\mathrm{M}=26.0 \mathrm{~ms}, \mathrm{SE}=5.0 \mathrm{~ms}$ ) and the control stimulation (Stroop effect: $\mathrm{M}=350$ $\mathrm{ms}, \mathrm{SE}=84 \mathrm{~ms}$ ) or only sham stimulated (Stroop effect: $\mathrm{M}=32.9 \mathrm{~ms}, \mathrm{SE}=7.2 \mathrm{~ms}$ ).

The active control did not significantly interact with either CCT $(Z=1.44, p=0.14)$ or interaction CCT x CPT $(Z=0.87, \mathrm{p}=0.38)$. 
Table 3: Statistical analysis of Experiment 2. The results of the GLMMs are shown for both accuracy and response time data of the second experiment. For every factor, the mean values and standard errors of each factor level are reported. The results of the statistical testing of the difference between these mean values are also reported as $\mathrm{Z}$ and $\mathrm{p}$ values.

\begin{tabular}{|c|c|c|c|}
\hline & Estimate (mean $\pm \mathrm{SE})$ & | Z value | & $\mathrm{p}$ value \\
\hline \multicolumn{4}{|l|}{ Accuracy (\%) } \\
\hline CCT & $\Delta 0.9$ & 2.952 & $<0.01$ \\
\hline Congruent & $98.0 \pm 0.5$ & & \\
\hline Incongruent & $97.1 \pm 0.6$ & & \\
\hline CPT & $\Delta 0.1$ & 0.441 & 0.65 \\
\hline Congruent & $98.6 \pm 0.6$ & & \\
\hline Incongruent & $98.7 \pm 0.4$ & & \\
\hline \multicolumn{4}{|l|}{ Stimulation } \\
\hline Sham & $98.5 \pm 0.3$ & & \\
\hline $6 \mathrm{~Hz}$ (vs. sham) & $\Delta 0.2$ & 0.579 & 0.56 \\
\hline $6 \mathrm{~Hz}$ & $98.3 \pm 0.5$ & & \\
\hline 9.7 Hz (vs. sham) & $\Delta 0$ & 0.43 & 0.66 \\
\hline $9.7 \mathrm{~Hz}$ & $98.5 \pm 0.4$ & & \\
\hline \multicolumn{4}{|l|}{ Response times (ms) } \\
\hline CCT (Stroop effect) & $\Delta 31.3$ & 3.12 & 0.001 \\
\hline Congruent & $561.1 \pm 15.0$ & & \\
\hline Incongruent & $592.4 \pm 18.4$ & & \\
\hline CPT & $\Delta 7.2$ & 2.28 & 0.02 \\
\hline Congruent & $573.1 \pm 16.5$ & & \\
\hline Incongruent & $580.3 \pm 16.6$ & & \\
\hline \multicolumn{4}{|l|}{ Stimulation } \\
\hline Sham & $585.1 \pm 24.1$ & & \\
\hline $6 \mathrm{~Hz}$ (vs. sham) & $\Delta 4.6$ & 0.78 & 0.43 \\
\hline $6 \mathrm{~Hz}$ & $580.5 \pm 21.8$ & & \\
\hline $9.7 \mathrm{~Hz}$ (vs. sham) & $\Delta 2$ & 0.06 & 0.95 \\
\hline $9.7 \mathrm{~Hz}$ & $584.9 \pm 27.5$ & & \\
\hline \multicolumn{4}{|l|}{ CCT $\mathrm{x}$ stimulation } \\
\hline Stroop effect (Sham) & $32.9 \pm 7.2$ & & \\
\hline Stroop effect (6 Hz vs. sham) & $\Delta 6.9$ & 2.11 & 0.03 \\
\hline Stroop effect $(6 \mathrm{~Hz})$ & $26.0 \pm 5.0$ & & \\
\hline Stroop effect (9.7 Hz vs. sham) & $\Delta 2.1$ & 1.44 & 0.14 \\
\hline Stroop effect $(9.7 \mathrm{~Hz})$ & $35.0 \pm 8.4$ & & \\
\hline CCT x CPT & $\Delta 11.8$ & 3.48 & $<0.001$ \\
\hline Stroop effect (CPT: congruent) & $36.7 \pm 7.6$ & & \\
\hline Stroop effect (CPT: incongruent) & $24.9 \pm 5.0$ & & \\
\hline \multicolumn{4}{|l|}{ CCT $x$ CPT $x$ stimulation } \\
\hline CCT x CPT (6 Hz vs. sham) & $\Delta 0.7$ & 0.35 & 0.72 \\
\hline CCT x CPT (9.7 Hz vs. sham) & $\Delta 1.8$ & 0.87 & 0.38 \\
\hline
\end{tabular}

SE: Standard error. 
$\operatorname{Exp} 1)$
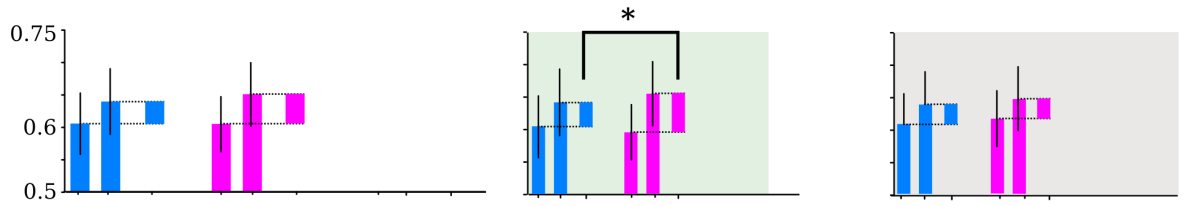

$\operatorname{Exp} 2)$
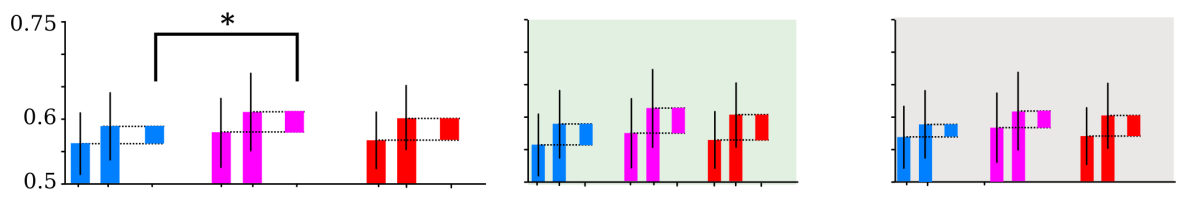

CCT: $\mathrm{c}=$ congruent CCT: $\mathrm{i}=$ incongruent Stroop effect: $\mathrm{c}-1$ $6 \mathrm{~Hz}$ Sham 9.7Hz

CPT: congruent $\mathrm{CPT}$ : incongruent

Combined data
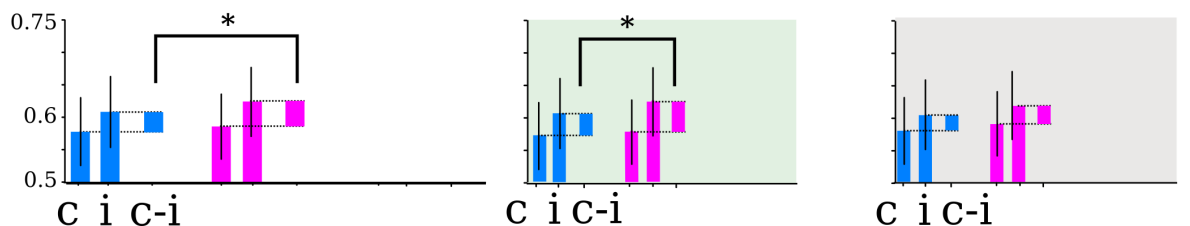

C i C-i

Figure 6: Effect of stimulation on response time. The response times for congruent and incongruent trials and the time difference between these (Stroop effect) are plotted for each stimulation condition individually for all trials in the left panels. In the middle panels, only data of trials which were preceded by a congruent trial are displayed; in the right panels, only for trials preceded by an incongruent trial. Experiment 1 (first row): CCT (size of Stroop effect) and stimulation interact significantly in trials preceded by congruent trials. Experiment 2 (middle row): the interaction CCT $\mathrm{x}$ stimulation is significant for all trials but not for the data subsets differentiated by the preceding trial. The active control stimulation in the alpha range did not change the interaction between stimulation and CCT. Combined dataset of both experiments (last row): the interaction between stimulation and CCT is significant across all trials. The significant interaction for trials preceded by congruent trials underlies the effect across all trials. All data is plotted including the $95 \%$ confidence interval.

\subsubsection{Joint analysis of both datasets}

he response time datasets of Experiments 1 and 2 were combined post hoc and reanalysed to increase statistical power (see Table 4). The control condition of Experiment 2 was excluded from further analysis, but the session order was included as a random factor in order to account for increased training effects not balanced out. The analysis of the response times revealed significant main effects for $\mathrm{CCT}(\mathrm{Z}=3.98, \mathrm{p}<0.001)$ but neither for stimulation $(\mathrm{Z}=1.25, \mathrm{p}=$ $0.20)$ nor for $\mathrm{CPT}(\mathrm{Z}=1.17, \mathrm{p}=0.23)$. For $\mathrm{CCT}$, the response times were faster for congruent $(\mathrm{M}=579.2 \mathrm{~ms}, \mathrm{SE}=12.0 \mathrm{~ms})$ compared to incongruent $(\mathrm{M}=6133 \mathrm{~ms}, \mathrm{SE}=140 \mathrm{~ms})$ trials

(Stroop effect). The significant interaction CCT x CPT $(Z=4.40, p<0.001)$ constitutes the Gratton effect, in which the size of the Stroop effect depends on whether the CCT is preceded 
by a congruent (Stroop effect: $\mathrm{M}=411 \mathrm{~ms}, \mathrm{SE}=50 \mathrm{~ms}$ ) or an incongruent (Stroop effect: $\mathrm{M}=$ $26.3 \mathrm{~ms}, \mathrm{SE}=3.5 \mathrm{~ms})$ trial. The interaction CCT $\mathrm{x}$ stimulation met the significance criterion $(\mathrm{Z}$ $=2.37, \mathrm{p}=0.01$ ), but the triple interaction CCT $\mathrm{x}$ stimulation $\mathrm{x}$ CPT did not meet the criterion $(\mathrm{Z}=1.37, \mathrm{p}=0.17$; see Figure 6$)$. The size of the Stroop effect depended on whether the participants are really stimulated (Stroop effect: $\mathrm{M}=29.5 \mathrm{~ms}, \mathrm{SE}=3.2 \mathrm{~ms}$ ) or sham-stimulated (Stroop effect: $\mathrm{M}=38.9 \mathrm{~ms}, \mathrm{SE}=5.1 \mathrm{~ms}$ ). Further exploration by dividing the dataset according to the congruency of the previous trial revealed a significant interaction CCT $\mathrm{x}$ stimulation for trials preceded by a congruent trial $(Z=2.65, \mathrm{p}<0.01)$ but no interaction if preceded by an incongruent one $(Z=0.71, p=0.47$; see Figure 6$)$. Thus, in trials preceded by congruent trials, the stimulation reduces the Stroop effect $(\mathrm{M}=33.3 \mathrm{~ms}, \mathrm{SE}=4.3 \mathrm{~ms})$ compared to sham $(\mathrm{M}=$ $48.9 \mathrm{~ms}, \mathrm{SE}=7.0 \mathrm{~ms}$ ).

\subsubsection{Diffusion drift model for conflict task}

In Experiment 1, Fisher-Pitman permutation tests investigated statistical differences in the DMC parameter (a, Ter, sr, $\mu_{c}, \zeta, \mathrm{t}_{\max }$, and $\left.\mathrm{t}_{90 t h}\right)$ samples recovered for either stimulation conditions. After correcting for multiple comparisons, no statistically significant difference was found for the parameters a $(Z=0.02, p=1), \mu_{c}(Z=0.51, p=1)$, Ter $(Z=0.22, p=1), s_{t}(Z=1.94$, $\mathrm{p}=0.33), \zeta(\mathrm{Z}=0.07, \mathrm{p}=1), \mathrm{t}_{90}(\mathrm{Z}=-0.38, \mathrm{p}=0.71)$, and $\mathrm{t}_{\max }(\mathrm{Z}=-0.12, \mathrm{p}=1)$. In Experiment 2, we employed Kruskal-Wallis tests to conduct statistical hypothesis testing on the DMC parameters recovered for all three stimulation conditions. No post hoc tests were performed as there was no statistically significant difference for all parameters after correcting for multiple comparisons: a $(\operatorname{maxT}=0.41, \mathrm{p}=1), \mu_{c}(\operatorname{maxT}=0.99, \mathrm{p}=1), \operatorname{Ter}(\operatorname{maxT}=0.38$, $\mathrm{p}=1), \mathrm{s}_{t}(\operatorname{maxT}=1.39, \mathrm{p}=1), \zeta(\operatorname{maxT}=0.93, \mathrm{p}=1), \mathrm{t}_{90}(\max \mathrm{T}=1.07, \mathrm{p}=0.71)$, and $\mathrm{t}_{\max }$ $(\operatorname{maxT}=1.18, \mathrm{p}=1)$.

\subsubsection{Arousal and sleep}

In the first experiment, the Wilcoxon signed rank test indicated no significant differences between stimulation conditions in mean arousal $(\mathrm{p}=0.878)$, sleep quality $(\mathrm{p}=0.999)$, and sleep duration $(\mathrm{p}=0.439)$. Similarly, neither the Kruskal-Wallis test indicated significant differences 
Table 4: Statistical analysis of combined dataset. The results of the GLMMs are shown for both accuracy and response time data of the combined dataset of both experiments. Additionally, the response times were divided according to the congruency of the previous trial in additional model runs. For every factor, the mean values and standard errors of each factor level are reported. The results of the statistical testing of the difference between these mean values are also reported as $\mathrm{Z}$ and $\mathrm{p}$ values.

\begin{tabular}{|c|c|c|c|}
\hline & Estimate (mean $\pm \mathrm{SE})$ & | Z value | & $\mathrm{p}$ value \\
\hline \multicolumn{4}{|l|}{ Response times (ms) } \\
\hline CCT (Stroop effect) & $\Delta 34.1$ & 3.98 & $<0.001$ \\
\hline Congruent & $579.2 \pm 12.0$ & & \\
\hline Incongruent & $613.3 \pm 14.0$ & & \\
\hline CPT & $\Delta 3.7$ & 1.17 & 0.23 \\
\hline Congruent & $595.1 \pm 25.5$ & & \\
\hline Incongruent & $598.8 \pm 25.5$ & & \\
\hline Stimulation & $\Delta 12.3$ & 1.25 & 0.2 \\
\hline Sham & $603.1 \pm 20.3$ & & \\
\hline $6 \mathrm{~Hz}$ & $590.8 \pm 19.3$ & & \\
\hline CCT $\mathrm{x}$ stimulation & $\Delta 9.4$ & 2.37 & 0.01 \\
\hline Stroop effect (Sham) & $38.9 \pm 5.1$ & & \\
\hline Stroop effect $(6 \mathrm{~Hz})$ & $29.5 \pm 3.2$ & & \\
\hline CCT x CPT & $\Delta 14.8$ & 4.40 & $<0.001$ \\
\hline Stroop effect (CPT: congruent) & $41.1 \pm 5.0$ & & \\
\hline Stroop effect (CPT: incongruent) & $26.3 \pm 3.5$ & & \\
\hline CCT x CPT x stimulation & $\Delta 13.3$ & 1.37 & 0.17 \\
\hline \multicolumn{4}{|c|}{ Response times (ms) - data divided according to congruency of previous trial (CPT) } \\
\hline \multicolumn{4}{|l|}{$\mathrm{CPT}=$ congruent } \\
\hline CCT $\mathrm{x}$ stimulation & $\Delta 15.6$ & 2.65 & $<0.01$ \\
\hline Stroop effect (Sham) & $48.9 \pm 7.0$ & & \\
\hline Stroop effect $(6 \mathrm{~Hz})$ & $33.3 \pm 4.3$ & & \\
\hline \multicolumn{4}{|l|}{$\mathrm{CPT}=$ incongruent } \\
\hline CCT $\mathrm{x}$ stimulation & $\Delta 2.3$ & 0.71 & 0.47 \\
\hline Stroop effect (Sham) & $27.9 \pm 5.7$ & & \\
\hline Stroop effect $(6 \mathrm{~Hz})$ & $25.6 \pm 3.9$ & & \\
\hline
\end{tabular}


between stimulation conditions in mean arousal $(\mathrm{p}=0.777)$, sleep quality $(\mathrm{p}=0.5278)$, and sleep duration $(\mathrm{p}=0.935)$. No subsequent pairwise comparisons were performed. Table S2 summarizes the descriptive and inferential statistics.

\subsection{Discussion}

Response conflict increases midfrontal theta dynamics between dACC and the DLPFC (Hanslmayr et al., 2008; Oehrn et al., 2014). We entrained the cortical control network exogenously by theta tACS during the Stroop task in order to support a causal role of this theta rhythm. For the combined data of both experiments, applying $6 \mathrm{~Hz}$ theta tACS reduced the Stroop effect significantly. This effect was driven as expected by reduction in Stroop effect only in trials preceded by congruent trials. The DMC parameters being unchanged did not allow for a more specific characterization.

This is in line with a reduction of congruency effect in the Simon task by theta tACS targeted towards the dACC (van Driel et al., 2015). This was driven by prolonged response times in congruent trials preceded by congruent trials $(\mathrm{cC})$. Our results and this study provide common evidence for a causal role of medio-frontal theta dynamics in cognitive control.

\subsubsection{Electrophysiology of the Stroop task}

EEG recordings in healthy subjects and intracranial recording in epilepsy patients suggest a causal role for the neural oscillatory connection between dACC and DLPFC (Hanslmayr et al., 2008; Oehrn et al., 2014). The power of theta oscillations is suggested to increase in proportion to the amount of response conflict (Hanslmayr et al., 2008). Independent of this theta power increase, the phase-coupling in theta range between left DLPFC and ACC changes depending on the congruency of the trial. Specifically, it persists longer and is stronger in incongruent trials (Hanslmayr et al., 2008). Additionally, DLPFC activity during the Stroop task is associated with activity increase in gamma frequency range $(30 \mathrm{~Hz}-100 \mathrm{~Hz})$, and electrical stimulation in this frequency band led to causal changes in performance (Koga et al., 2011). Intracranial EEG recordings revealed for the DLPFC a preparatory period directly after stimulus offset in which theta power increases and gamma oscillations are coupled to theta oscillations (Oehrn et al., 2014). This cross-frequency coupling correlates with accuracy. The detection of conflict at 
around $290 \mathrm{~ms}$ leads to increases in theta power in dACC, which drives the phase-coupling in theta range with the DLPFC and cross-frequency coupling between DLPFC gamma activity and the phase of the theta activity in AACC. Also, the theta power in dACC between conflict detection and resolution correlates positively with response time. The dACC modulates the DLPFC activity before conflict resolution, whereas the DLPFC modulates the dACC after conflict resolution. The modulation occurs via theta phase synchronization. The gamma power in DLPFC after a response correlates negatively with response times for the next incongruent trial reflecting a preparatory mechanism, and the increased theta phase synchronization is a mechanism for the DLPFC to influence the dACC theta activity. Thus, the directionality of information transfer from DLPFC to dACC via theta phase synchronization and gamma activity cross-frequency coupling (CFC) might implement a different response strategy, which does not require the dACC to be active (Oehrn et al., 2014).

We chose the DLPFC as a target since its location at the brain surface allows a more reliable stimulation. Stimulation of the dACC, in which due to its deep location is a more difficult target, has been done however (van Driel et al., 2015; Onoda et al., 2017; To et al., 2018). Active conflict detection and resolution are attributed to dACC activity, whereas the adaptation after response is attributed to DLPFC activity (Oehrn et al., 2014). Increased DLPFC activity after incongruent trials leads to less dACC activity following conflict trials and to reduced congruency effects (Silton et al., 2010). Exogenously increased DLPFC activity would reduce dACC activity similarly. Since we targeted the DLPFC with tACS, we hypothesized that the DLPFC-dACC circuitry might have been preferentially influenced when DLPFC was active in adaptation of cognitive control for the next trial. Event-related tACS only during the conflict detection and resolution phase in which the $\mathrm{dACC}$ is driving the interaction or only during the adaptation phase could lead to different behavioral outcomes as the latter might have a higher efficacy in manipulating the circuitry's activity. It remains an open question if the DLPFC-dACC circuitry can only be modulated intermittently when stimulating the DLPFC constantly.

It has to be noted that in the first experiment the reduced congruency effect was clearly driven by trials which were preceded by congruent trials. It fits very well in our second hypothesis that stronger cognitive control is exerted when tACS increases the normally low DLPFC activity. However, the Stroop effect was reduced for all trials in the second experiment, not only those preceded by congruent trials. Therefore, the first hypothesis that theta-range tACS reduces the 
Stroop effect is fulfilled. While $6 \mathrm{~Hz}$ tACS reduced the Stroop effect in both experiments, it is a partial replication as different subsets of data are affected. In the combined dataset of the studies, both effects survive the joint analysis, showing a general effect of DLPFC on Stroop effect across all participants. Both experiments were designed equally except for the active control condition. Participants acted as their own control by participating in all sessions of an experiment, which cancels out possible difference in performance between the experiments. Therefore, the pooling of the data of both experiments is statistically valid and allows the interpretation of trends underlying both datasets. Inconsistent effects of tACS have been reported before in internal replications (Veniero et al., 2017), but the results of both experiments in this study causally corroborate the importance of DLPFC activity during the Stroop task.

We confirmed the validity of the used DMC by recovering simulated data (see Supplementary Material), replicating an earlier study (White et al., 2018). We hypothesized the DMC parameters to reflect increased conflict adaptation and therefore a decreased influence of the automatic process on the stochastic decision process. This would entail a combination of reduced amplitude and reduced $t_{90}$ or $t_{\max }$ of the automatic process (word reading), which we did not find when estimating the parameters (Ulrich et al., 2015). Therefore, the DMC models indicate for both experiments that the influence of the automatic process remained the same for sham and active stimulation. In the first experiment, $6 \mathrm{~Hz}$ stimulation reduced the Stroop effect for all trials by $12.4 \mathrm{~ms}(38.8 \%)$ compared to sham; in the second experiment, by $6.9 \mathrm{~ms}(22.0 \%)$. The breakdown of the behavioral data of each participant and session into 7 underlying DMC parameters reduced the statistical power of the subsequent analysis. Therefore, the DMC was insufficient to detect small absolute changes in both response times and accuracy in this study.

The stimulation frequency of $6 \mathrm{~Hz}$ chosen as oscillatory power in narrow-band theta $(6 \mathrm{~Hz}-$ $7 \mathrm{~Hz}$ ) in the left-frontal region correlates with reaction time in conflict adaptation (Tang et al., 2013). While most studies documenting the increased phase-coupling between DLPFC and $\mathrm{dACC}$ do not further delimit the frequency beyond being in the theta range, it has been suggested that dACC theta phase at $5 \mathrm{~Hz}$ modulates gamma activity in the DLPFC (Oehrn et al., 2014). As the frequency of $6 \mathrm{~Hz}$ is at the center of the range of individualized theta frequencies in a study employing a Simon task, we are confident that $6 \mathrm{~Hz}$ stimulation was an appropriate choice (van Driel et al., 2015). In particular, in the context of theta-gamma coupling, the option of superimposing more gamma cycles on a longer theta wave may provide better effects in 
future experiments. Further studies might obtain stronger abolishments of the Stroop effect when stimulating at the individuals' theta peak frequencies (Alekseichuk et al., 2016).

Our choice of active control frequency in the second experiment fell on a nonharmonic frequency in the alpha range. In previous studies, alpha power decreased after conflict trials as it marks higher arousal (Tang et al., 2013; Carp and Compton, 2009) but has no indicated role in conflict detection or resolution.

Alpha tACS showed a trend towards a reduced congruency effect during the Simon task in an earlier study (van Driel et al., 2015). Our results do not show this trend, and therefore, the effect of theta tACS on the conflict processing cannot be attributed to unspecific stimulation effects.

\subsubsection{Outlook and clinical relevance}

For future studies, the stimulation of the DLPFC in a broad gamma range would be a promising target as DLPFC gamma power after response predicted response times in subsequent trials. Also, theta-gamma cross-frequency stimulation paradigms promise stronger abolishment of the Stroop effect as they effectively change functionality of distant brain regions which exhibited this type of cross-frequency behavior (Alekseichuk et al., 2016).

It is of note that the theta stimulation to the DLPFC could be equally effective if limited to the time after response. Therefore, the effect of stimulation on adaptation could be isolated while not interfering with conflict detection and resolution. A transfer of the stimulation paradigm to different conflict tasks could show causally if the cognitive control network's physiology is equal in all these tasks. The Stroop task is a frequently applied neurophysiological test to study neural mechanisms of inhibitory control and its dysfunction (Strauss et al., 2006). Clinically diverse disorders as chronic alcoholism, schizophrenia, and age-related memory impairment are associated with increased interference in the Stroop task (Dao-Castellana et al., 1998; Hanes et al., 1996; Hänninen et al., 1997), which is a biomarker for the inability to correctly inhibit automatic responses and to maintain goal-directed behavior (Strauss et al., 2006). Both these executive functions are essential to living a well-adapted life, and their restoration is desirable (Diamond, 2013). 


\subsection{Conclusion}

This is the first study stimulating the DLPFC by theta tACS. We demonstrate that the cognitive control network can also be influenced by stimulation targeting the DLPFC. We were able to reduce the Stroop effect in a subset of trials over both experiments. The equalization of response times in congruent and incongruent trials suggests that postconflict adaptation was changed. We propose the hypothesis that theta stimulation of the DLPFC is effective in changing preparatory mechanisms after conflict resolution. The key questions to be clarified are whether (a) gamma tACS leads to more reduction of the Stroop effect, (b) theta stimulation applied only after a conflict resolution is equally effective, and (c) the results are generalizable to other conflict tasks.

\section{Data availability}

The behavioral data used to support the findings of this study are available from the corresponding author upon request.

\section{Disclosure}

The funders had no role in study design, collection and analysis of data, decision to publish, or drafting the manuscript.

\section{Conflicts of interest}

The authors declare that there is no conflict of interest regarding the publication of this paper.

\section{Acknowledgments}

We acknowledge support by the German Research Foundation and the Open Access Publication Funds of the Göttingen University. The study was funded by the University Medical Center Göttingen, Göttingen, Germany. 


\subsection{Supplementary material}

\section{Diffusion drift model for conflict task}
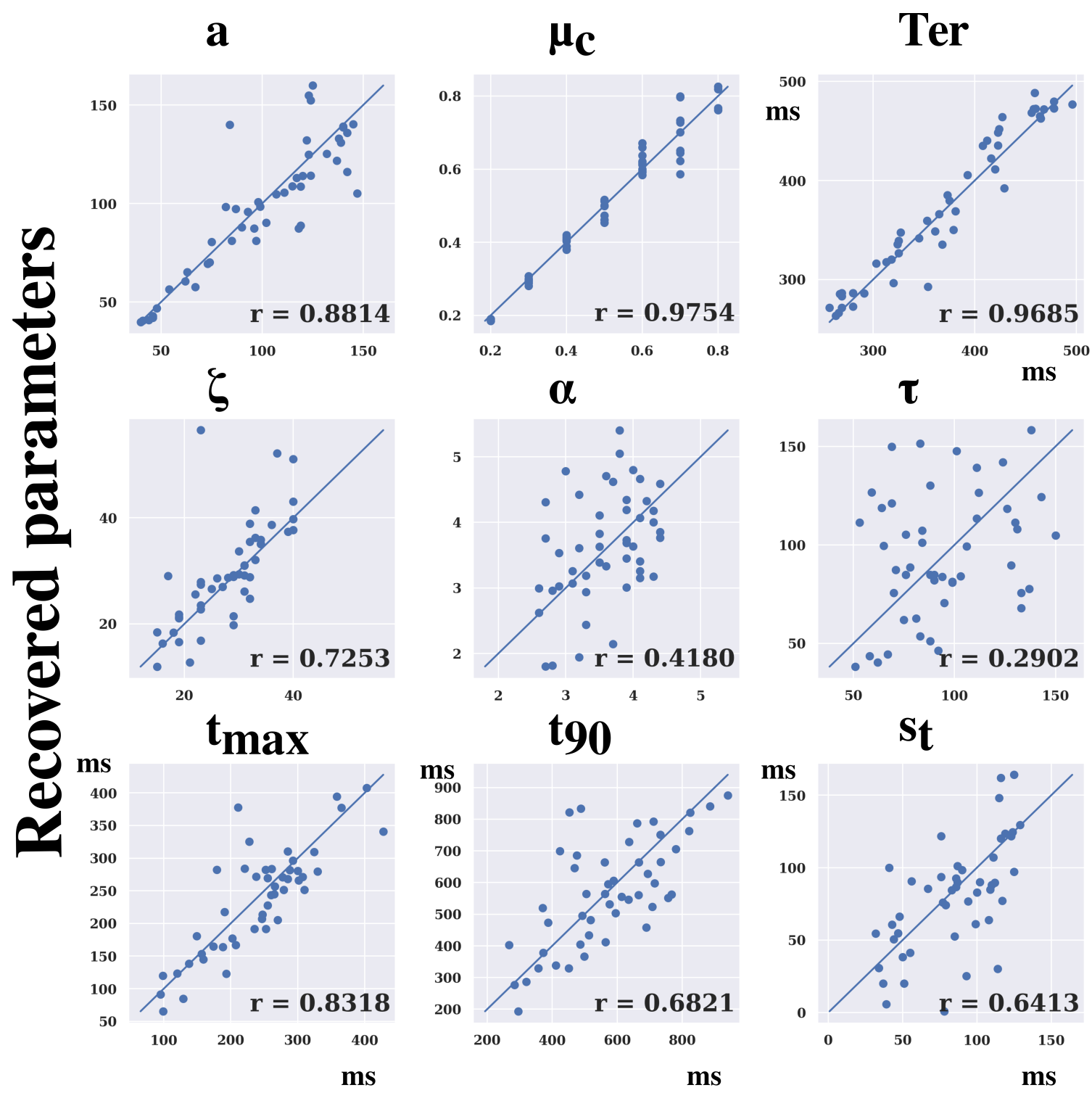

\section{Original parameters}

Figure 7: Correlation coefficients between the original and recovered parameters of the recovery study. Values close to the identity line indicate well-recovered parameter values. The parameters a (Boundary of decision process); $\mu_{c}$ (drift rate of controlled process); Ter (Non-decision time); $\zeta$ (peak amplitude), $\alpha$ (shape) and $\tau$ (characteristic time) for the automatic activation gamma function; $\mathrm{t}_{\max }$ (latency of the peak amplitude), $\mathrm{t}_{90}$ ( 0.9 quantile of automatic process gamma function) of the automatic process gamma function; $\mathrm{s}_{t}$ (variability of the non-decision time) are shown.

Recovery study for DMC DMC has been methodologically tested in a parameter-recovery study by White et al. (2018). To the best of our knowledge DMC has not been used with Stroop 
task data. We defined starting values for fitting based on pilot study results and conducted a similar parameter-recovery study for 48 data sets of both 300 simulated congruent and incongruent trials. In the recovery study the average G2 statistic across all 48 datasets was 6.9 , indicating good model fits. In the first experiment the average G2 statistic was 23.2, in the second experiment, 18.6

Data recovery Similar to White et al. (2018), we sampled the value of each DMC parameter from uniform distributions. The 'original data' was created with these underlying parameters, which were supposed to be recovered using the DMC. As the original parameter values are known, the quality of the DMC can be assessed by calculating the correlation coefficients $r$ between original and recovered data. For each parameter the recovery was judged to be recovered either poorly $(r<0.5)$, fairly $(0.5<r<0.75)$, well $(0.75<r<0.9)$ or excellently $(r>0.9)$.As shown in Figure S1, two parameters were recovered excellently, the drift rate of the controlled process $\left(\mu_{c}, r=0.9754\right)$ and the non-decision time (Ter, $\left.r=0.9685\right)$. The boundary of the decision process $(\mathrm{a}, \mathrm{r}=0.8814)$ and latency of the peak amplitude of the automatic process $\left(\mathrm{t}_{\max }\right.$ , defined as $\tau(\alpha-1), \mathrm{r}=0.8318)$ were well recovered, the variability of the non-decision time $\left(\mathrm{s}_{t}, \mathrm{r}=0.6413\right)$, the amplitude of the automatic process $(\zeta, \mathrm{r}=0.7253)$ and the 0.9 quantile of the automatic process gamma function ( $\left.\mathrm{t}_{90}, \mathrm{r}=0.6821\right)$ fairly. The shape $(\alpha, \mathrm{r}=0.4180)$ and the characteristic time $(\tau, 0.2902)$ of the describing the automatic process gamma function were excluded from further analysis as they are incoporated in $t_{\text {max }}$ and $t_{90}$ and as recovery was poor similar to the earlier recovery study (White et al., 2018). The seven parameters with fair or better recovery were used in a linear model to investigate changes in parameters induced by electrical stimulation.

\section{The age, gender, arousal and sleep of participants}

Table 5: Gender and age of participants in both experiments.

\begin{tabular}{lll}
\hline & $\mathrm{N}$ & Mean age $( \pm \mathrm{SD})$ \\
\hline Experiment 1 & & $24.4 \pm 3.8$ years \\
Women & 8 & \\
Men & 2 & \\
Experiment 2 & & $25 \pm 3.07$ years \\
Women & 8 & \\
Men & 4 &
\end{tabular}


Table 6: Arousal and Sleep indicators. The non-parametric paired samples Wilcoxon signed rank test was used for hypothesis testing in Experiment 1, the non-parametric paired sample Kruskal- Wallis test in Experiment 2.

\begin{tabular}{|c|c|c|c|}
\hline & Condition & Mean $\pm \mathrm{SD}$ & Test (p-value) \\
\hline \multicolumn{4}{|c|}{ Mean Arousal (1 - 10; max: 10) } \\
\hline \multirow[t]{2}{*}{ Experiment1 } & $6 \mathrm{~Hz}$ & $5.6 \pm 2.3$ & 0.878 \\
\hline & Sham & $5.85 \pm 1.5$ & \\
\hline \multicolumn{4}{|l|}{ Experiment 2} \\
\hline & $6 \mathrm{~Hz}$ & $6.3 \pm 1.8$ & 0.777 \\
\hline & $9.7 \mathrm{~Hz}$ & $6.6 \pm 1.5$ & \\
\hline & Sham & $6.2 \pm 1.5$ & \\
\hline \multicolumn{4}{|c|}{ Sleep quality ( $1-5$; best: 5$)$} \\
\hline \multicolumn{4}{|l|}{ Experiment1 } \\
\hline & $6 \mathrm{~Hz}$ & $3.9 \pm 0.7$ & 0.999 \\
\hline & Sham & $3.8 \pm 0.9$ & \\
\hline \multicolumn{4}{|l|}{ Experiment 2} \\
\hline & $6 \mathrm{~Hz}$ & $4.0 \pm 0.9$ & 0.527 \\
\hline & $9.7 \mathrm{~Hz}$ & $4.0 \pm 0.9$ & \\
\hline & Sham & $3.6 \pm 1.0$ & \\
\hline \multicolumn{4}{|c|}{ Sleep quality ( $1-5$; best: 5$)$} \\
\hline \multicolumn{4}{|c|}{ Experiment1 } \\
\hline & $6 \mathrm{~Hz}$ & $7.3 \pm 0.6$ & 0.439 \\
\hline & Sham & $6.8 \pm 1.4$ & \\
\hline \multicolumn{4}{|l|}{ Experiment 2} \\
\hline & $6 \mathrm{~Hz}$ & $7.2 \pm 1.4$ & 0.935 \\
\hline & $9.7 \mathrm{~Hz}$ & $7.3 \pm 1.5$ & \\
\hline & Sham & $6.9 \pm 1.7$ & \\
\hline
\end{tabular}




\section{Theta transcranial alternating current stimulation decreases conflict effect in Stroop task}

Authors: Albert Lehr ${ }^{1}$, Micha Siegle ${ }^{1}$, Walter Paulus ${ }^{1}$ and Andrea Antal ${ }^{1}$

Affiliation: 'Department of Clinical Neurophysiology, University Medical Center Göttingen, Göttingen 37073, Germany

prepared for publication

Author contributions:

Detailed author contribution of AL: conceptualization, study design, project administration, methodology, software (behavioral paradigm), data collection, supervision of data collection, supervised medical student MS, formal analysis, prepared all illustrations, data visualization, interpreted data, data curation and wrote original draft.

MS: study design, data collection

WP: resources and funding acquisition

AA: project administration, supervised medical student MS, contributed to writing original draft. 


\subsection{Abstract}

Cognitive control is the ability to adapt cognitive processes based on task demands. Cognitive control arises from an in intricate network when competing cognitive processes are in competition. The network then promotes the resolution of this conflict. Previous electrophysiological studies have linked cognitive control to neural oscillations in the theta band arising from the dorsal anterior cingulate cortex (dACC), which couples intimately with the dorsolateral prefrontal cortex.. Here, we studied the behavioral and neurophysiological effects transcranial alternating current stimulation (tACS) and transcranial random noise stimulation (tRNS). 24 healthy participants received four transcranial electrical stimulation protocols in a double-blind, randomized, repeated-measures study design. $6 \mathrm{~Hz}$ tACS and tRNS were hypothesized to be modulators of activity in dACC during conflict with $4 \mathrm{~Hz}$ tACS being an active control. Additionally, sham stimulation served as an inactive control to all three active stimulation protocols. During stimulation participants performed a conflict-inducing color-word Stroop task that activates the dACC among others. Before and after the stimulation EEG was measured during the same task. Generalized linear mixed model analyses revealed that $6 \mathrm{~Hz}$ tACS reduced the Stroop effect during stimulation compared to sham, while $4 \mathrm{~Hz}$ tACS and tRNS did not. The behavioral effects did not persist after stimulation. Hypothesis-driven EEG analysis revealed no clear difference in non-phase locked midline theta range power $400 \mathrm{~ms}$ to $700 \mathrm{~ms}$ after stimulus presentation. Our results suggest that mid-theta range neural oscillations are causally connected to the Stroop effect and therefore to cognitive control.

\subsection{Introduction}

Cognitive control selects appropriate cognitive processes and behaviors according to current demands (Miller and Cohen, 2001). Also, any interfering information that may trigger inappropriate actions is suppressed. A distinct cognitive control network $(\mathrm{CCN})$ is responsible for detecting conflicts, favouring appropriate behaviors and monitoring their implementation until goal achievement (Cole and Schneider, 2007). The dorsolateral prefrontal cortex (DLPFC), the dorsal anterior cingulate cortex (dACC) and the posterior parietal cortex (PPC) are most commonly recognized as key hubs of the network (Chein and Schneider, 2005; Dosenbach et al., 2006). 
As cognitive control is much needed in a world full of distractions, the network has been studied in a multitude of tasks (e.g. Stroop (1935); Simon and Rudell (1967); Eriksen and Eriksen (1974); Cavanagh et al. (2013)). Famous for its extensive use in neurophysiological diagnostics, the Stroop color-word task (SCWT) pits long-trained reading against seldomly-used color naming (Stroop, 1935). When seeing a color, extracting the semantic meaning is a habituated, effortless behavior, while naming the ink color requires effort. Participants have to name the ink color accurately and as quickly as possible. No conflict arises when both semantic meaning and the ink color match (low-conflict, congruent, e.g. "RED" in red ink). Actually, the match will lead to shorter response times (RT), as the habituated behavior facilitates the correct response. However, conflict arises when the semantic meaning and the ink color do not match (highconflict, incongruent, e.g. "RED" in green ink). Thus, the habituated behavior interferes with task completion. The Stroop effect is defined as the difference in RT between the facilitation and the interference (MacLeod, 2005).

Midfrontal theta-band activity $(4-8 \mathrm{~Hz}$ ) correlates with conflict processing (Hanslmayr et al., 2008; Nigbur et al., 2011; Oehrn et al., 2014). The dACC as part of the medial frontal cortex most likely generates this oscillations at around $6 \mathrm{~Hz}$ (Hanslmayr et al., 2008; Oehrn et al., 2014). Theta-phase coupling with the DLPFC has been shown to increase in high-conflict during Stroop task (Hanslmayr et al., 2008; Oehrn et al., 2014). Theta-band oscillation power not locked to stimulus or response robustly correlates with RT (Cohen and Donner, 2013). The mechanistically plausible hypothesis that inter-areal theta activity underlies the implementation of cognitive control is strengthened mostly by correlative evidence (Cohen, 2014a).

One technique to infer the causal role of brain oscillations and cognitive processes is transcranial alternating current stimulation (tACS), which alters the activity in brain networks (Antal et al., 2008). It injects sinusoidally oscillating current into cortical tissue, which via modulation of neural membrane potential entrains neural networks (Ali et al., 2013; Helfrich et al., 2014). Indeed, stimulation within the theta range reduced the conflict effect in another cognitive control task (van Driel et al., 2015; Simon and Rudell, 1967).

Transcranial random noise stimulation (tRNS) also changes excitability in the cortex (Terney et al., 2008). tRNS might enhance neural activity in the long-term via its effect on sodium channels (Paulus, 2011). To our knowledge, tACS and tRNS has never been used to alter conflict processing by targeting the dACC during Stroop task. One general caveat of tACS and tRNS 
experiments is that EEG cannot be measured simultaneously (Kasten et al., 2016). The effects of tACS are, however, reported to persist up to $70 \mathrm{~min}$ after stimulation of the visual cortex (Kasten et al., 2016).

We derived our hypotheses from the observation that theta connectivity increases in incongruent trials (I) compared to congruent trials (C; Hanslmayr et al. (2008)). All trials after incongruent trials are therefore associated with (still) higher theta connectivity. Incongruent trials are followed either by congruent trials (iC) or by incongruent trials (iI). Interestingly, the difference in RT (i.e. Stroop effect) between iI and iC is smaller than normally between $\mathrm{C}$ and I. This adaptation will be referred to as conflict adaptation effect (Gratton et al., 1992). Thus, an incongruent trial modulates the Stroop effect in the following trial. Stronger Stroop effect adaptation correlates with midfrontal theta power (Pastötter et al., 2013). The higher the activity in the ACC, the greater the behavioral adaptation and the prefrontal cortex activity (Kerns et al., 2004).

The drift diffusion model for conflict tasks captures RT of the Stroop task (Ulrich et al., 2015). It is an extension of the influential drift-diffusion model for two alternative-forced choice tasks (Ratcliff, 1978; Ratcliff and McKoon, 2008). Based on the distribution of RT for both congruent and incongruent trials, it determines several parameters that characterize the decision process during Stroop task. Most importantly, the DMC parameter $\zeta$ describes the amplitude of confounding influence of the automatic process (reading the color word) on the response accuracy and time.

Is the increased theta connectivity in incongruent trials causing the conflict adaptation effect or is it an epiphenomenon of conflict processing? We hypothesized (1) that externally increasing theta connectivity by tACS would reduce the Stroop effect in RT compared with sham stimulation. Theta connectivity is low in $\mathrm{cI}$ and $\mathrm{cC}$ trials, because of the preceding low-conflict trial. Therefore, (2) the Stroop effect in RT is reduced preferentially in trials following an congruent trial. All results of transcranial stimulation will be compared to sham stimulation, which blinds volunteers and experimenters. (3) The effects of tACS will appear if the frequency is in midtheta range $(6 \mathrm{~Hz})$, but not in the slightly out-of-range $4 \mathrm{~Hz}$ that therefore will serve as an active control.

As EEG concurrently with transcranial stimulation is technically not feasible (Helfrich et al., 2014; Kohli and Casson, 2019), we measured task-based EEG before and after the stimulation phase as a proxy. Thus, we hypothesize that non-phase locked theta power increases persist in 
the post-stimulation EEG. Most importantly, (4) the difference in theta power between congruent and incongruent trials will be reduced after $6 \mathrm{~Hz}$ stimulation compared to after sham stimulation. Persistent behavioral changes are then associated with (5) the same behavioral effects on RT as in the main stimulation phase only for $6 \mathrm{~Hz}$ stimulation (see hypothesis 1 and 2 ).

Furthermore, we hypothesize that the influence of the automatic process on the Stroop task is reduced during and after $6 \mathrm{~Hz}$ tACS. Thus, (6) the DMC parameter $\zeta$ describing amplitude of the automatic process should be reduced as cognitive control increases.

\subsection{Methods}

\subsubsection{Participants}

27 German-speaking adult volunteers consented in writing to join the study. 24 (age range: 19 32, 12 females) completed the study. Three did not complete all four sessions due to individual reasons. They were informed about possible adverse effects of tES and the inclusion criteria, which were right-handedness (Oldfield, 1971), normal or corrected-to-normal vision, no use of medication acting on the central nervous system and absence of self-reported neurological, psychiatric or substance use disorders. This study was conducted according to the regulations of the 1964 Declaration of Helsinki and approved by the ethics committee of the University Medical Center of Goettingen, Germany.

\subsubsection{Experimental protocol}

This study was placebo-controlled and double-blind. All subjects participated in all four sessions during which tACS $(4 \mathrm{~Hz}, 6 \mathrm{~Hz})$, tRNS or sham stimulation was applied. The stimulation condition order was counterbalanced, equalizing possible learning effects. The washout period between experiments was $48 \mathrm{~h}$, reducing possible carryover effect. The participants reported prior night's sleep quality and length, their bodily experience of the stimulation and their arousal at beginning and end of the session.

\subsubsection{Color-word Stroop task}

In this color-word Stroop task (CWST) participants had to indicate the font color of the four German color words (GREEN, RED, PURPLE, BLUE) (Stroop, 1935). The font of the color 
words could appear in any of the four colors, thus the font color either matched the color word's meaning or not. The word BLUE written in blue constituted a congruent trial, GREEN written in blue an incongruent trial. The response options green and purple were mapped onto the same response button of a response pad (RB-740; Cedrus Corporation, San Pedro, USA). Similarly, red and blue shared a response button. However, in incongruent trials the meaning of the color word and the font color did not map onto the same button (e.g. RED was never shown in blue or GREEN in purple). Therefore, this was effectively a two-alternative forced-choice task. We used the CIE Lightness Chrome hue device-independent colorimetric space (Commission Internationale de l'éclairage, 1976). The four colors, Green (hue = 140), Red (hue = 40), Purple (hue $=320)$, Blue (hue $=240)$, had fixed lightness $(\mathrm{L}=51)$ and chroma level $(\mathrm{CL}=55 \%)$ to account for Piéron's law (Stafford et al., 2011). In each session the participants first practiced the Stroop task for at least 50 trials, in which feedback about the correctness of the response was provided. As soon as participants reached $80 \%$ accuracy within the last 20 trials, the training phase ended.

1)

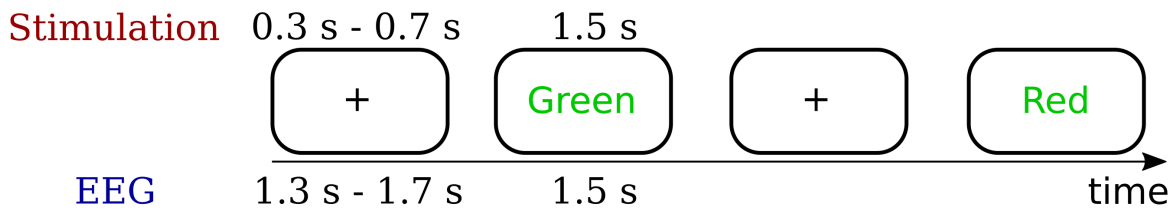

2)

\begin{tabular}{|c|c|c|c|c|c|}
\hline $\begin{array}{l}\text { Practice } \\
\sim 50 \text { trials }\end{array}$ & $\begin{array}{l}\text { 1st EEG } \\
192 \text { trials } \\
10 \mathrm{~min}\end{array}$ & $\overrightarrow{1 \mathrm{~min}}$ & $\begin{array}{l}\text { Stimulation } \\
608 \text { trials } \\
20 \mathrm{~min}\end{array}$ & $\overrightarrow{1 \mathrm{~min}}$ & $\begin{array}{l}\text { 2nd EEG } \\
192 \text { trials } \\
10 \text { min }\end{array}$ \\
\hline
\end{tabular}

Figure 8: The experimental procedure is shown for 1) individual trials and 2) for a complete session of the experiment. The interstimulus interval was longer during EEG measurement to facilitate the analysis.

In the first experimental phase, event-related EEG and behavioral responses were recorded during 192 trials. Stimulation was applied and the responses to 608 trials were recorded during the next phase. Finally, EEG and responses were again recorded during 192 trials. In the experimental phase no feedback was given. Congruent and incongruent trials were randomized and presented equally often. The length of a trial was $1.5 \mathrm{~s}$. The duration of the interstimulus 
interval was drawn from a uniform distribution between $0.3 \mathrm{~s}$ and $0.7 \mathrm{~s}$ for the stimulation phase and between $1.3 \mathrm{~s}$ and $1.7 \mathrm{~s}$ for the EEG phases. The interstimulus intervals varied to prevent phase-locking of stimulation to trial onset. Participants fixated a gray cross $(\mathrm{L}=51)$. The participants were instructed to respond as quickly as possible while maintaining perfect accuracy. The task was programmed in the PsychoPy toolbox (Peirce, 2007). The behavioral readout consists of RT and response accuracy.

\subsubsection{Transcranial electrical stimulation}

Stimulation was delivered by a CE-certified NeuroConn multichannel stimulator (neuroConn $\mathrm{GmbH}$, Ilmenau, Germany) throughout the stimulation phase. Being based on a previous study (To et al., 2018), the main electrode was positioned at Fz according to the international 10-10 EEG system with three return electrodes (AF7, Fpz, AF8; see Figure 9). The current intensity was $1 \mathrm{~mA}$ peak-to-baseline at the main electrode $(\mathrm{Fz})$ and $0.33 \mathrm{~mA}$ at each return electrode. The electrodes were round with a diameter of $2 \mathrm{~cm}$.

1)

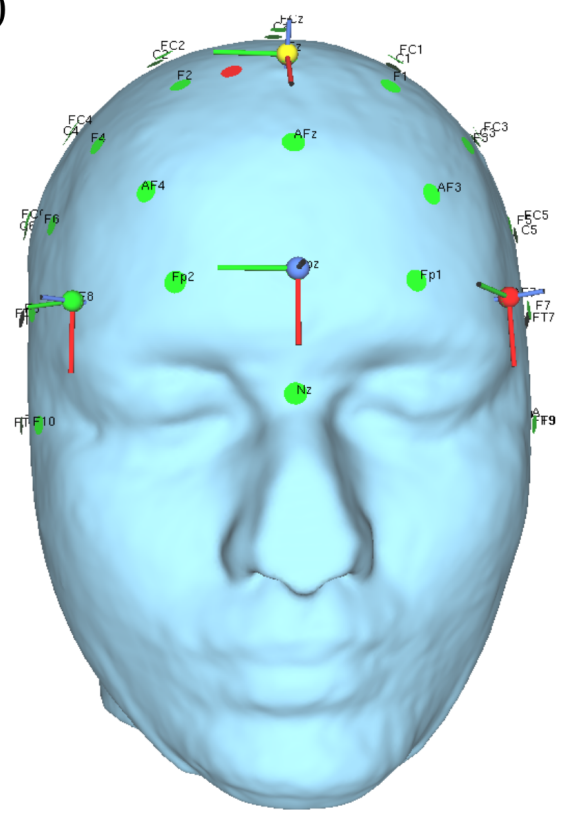

2)
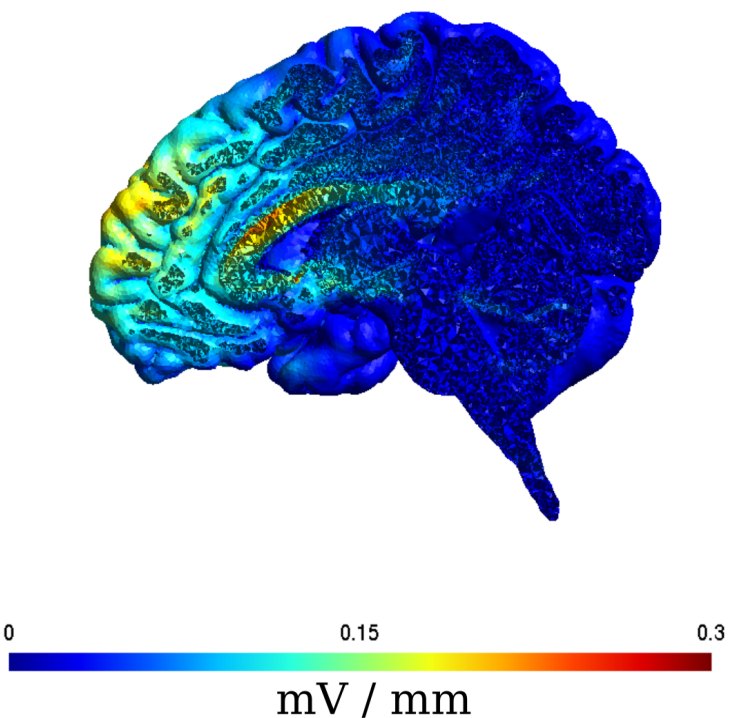

Figure 9: The tACS montage for stimulation of the dorsal anterior cingulate cortex (dACC) and the modelled electric field strength. 1) The main electrode of the montage is positioned over Fz. Three return electrodes at AF7, Fpz and AF8 each channel back a third of the current. 2) The electric field strength is $0.12 \mathrm{mV} / \mathrm{mm}$ over the dACC. The graphics and electric field strength modelling are derived from SimNIBS 3.0.2. 
Following the recommendations of previously published modelling studies (Saturnino et al., 2015), the orientation of the plugs and cables was kept constant (facing away perpendicular to the medial line). Round rubber electrodes of $1 \mathrm{~cm}$ radius were fixed on the scalp of participants with the conductive Ten20 paste. This placement leads to left hemispheric frontal stimulation with peak field intensities around $0.1 \mathrm{mV} / \mathrm{mm}$ according to simulations with SimNIBS standardized head model (Figure 9; Thielscher et al. (2015)). The four stimulation conditions consisted of two tACS sessions with either $4 \mathrm{~Hz}$ or $6 \mathrm{~Hz}$, white noise tRNS between $0 \mathrm{~Hz}$ and $1000 \mathrm{~Hz}$ and a sham stimulation limited to each $10 \mathrm{~s}$ long ramp-up and ramp-down periods at both the beginning and end of the stimulation session (Antal et al., 2008; Terney et al., 2008). The sham stimulation thereby had a inconsiderable effect on performance, but participants were blinded. Impedances were kept below $15 \mathrm{k} \Omega$.

\subsubsection{EEG recording}

The participants performed 192 trials of the CWST during the EEG recordings before and after the stimulation. Event-related EEG was acquired at a sampling rate of $1000 \mathrm{~Hz}$ and with DC (0 Hz) included with a NeuroConn NeuroPrax EEG device (NeuroConn, Illmenau, Germany). No bandpass or notch filter was applied during recording. The reference was placed at the right mastoid but the EEG activity was later rereferenced to common average. $28 \mathrm{Ag}$-AgCl electrodes were positioned at Fp1, Fp2, F7, F3, F4, F8, FC5, FC1, FC2, FC6, T3, C3, Cz, C4, T4, CP5, CP1, CP2, CP6, T5, P3, Pz, P4, T6, O1, O2. Impedances were kept below $20 \mathrm{k} \Omega$. Two EOG channels were used to send electrical trigger inputs from the presentation software PsychoPy and demarcate fixation and trial periods. The data were epoched between $1 \mathrm{~s}$ before and $1.7 \mathrm{~s}$ after stimulus presentation. Epochs of error trials and of post-error trials were removed. The epochs were demeaned over the complete duration and detrended. Epochs with high variance or high kurtosis were removed automatically before electrical and muscular artifact-containing epochs were eliminated manually. Eye blinks and lateral eye movements were eliminated using independent component analysis (Jung et al., 1998). The EEG data preprocessing was done using the FieldTrip toolbox (Oostenveld et al., 2011).

\subsubsection{Analysis}

All analyses were conducted in the R software (R Core Team, 2018) if not stated otherwise. 
3.3.6.1 Sleep and arousal At the beginning and end of each experimental session the participants self-reported their arousal on a scale from one (tired) to ten (completely awake). Similarly, they reported sleep quality in the prior night on a scale from 1 (bad) to 5 (good). Higher arousal levels increase error rates in incongruent trials (Pallak et al., 1975), while sleep deprivation generally increases RT without changing interference or facilitation (Cain et al., 2011). Both indicators were individually compared across stimulation conditions employing a non-parametric paired sample Kruskal-Wallis test.

3.3.6.2 Generalized linear mixed models for behavioral data Generalized linear mixed models (GLMM) are used in research designs in which not normally distributed data from multiple non-independent data sources is analyzed (Breslow and Clayton, 1993). We analyzed all GLMM with the package lme41.1-15 in R (Bates et al., 2014). During response time tasks multiple participants respond many times, creating datasets in which each participant has a unique data distribution. The RT are analyzed without averaging per participant, but acknowledging the individual participants as non-independent data sources, thus increasing the statistical power. Additionally, RT distributions have a right skew as responses can be given until trial termination (1.5 s after stimulus presentation) and necessarily are of a positive value. An inverse Gaussian distribution was used to account for this distribution (Lo and Andrews, 2015). Participants and word-color per trial were included as random effects in all GLMM for behavioral data. Thus, individual differences in speed are accounted for the across-person variability.

For the GLMM of the phase during which transcranial stimulation was applied the categorical fixed factors congruency of current trial (CCT; factor levels: congruent, incongruent), congruency of previous trial (CPT; factor levels: congruent, incongruent) and stimulation (factor levels: sham, $4 \mathrm{~Hz}$ tACS, $6 \mathrm{~Hz}$ tACS, tRNS) were sum-coded. For the fixed effect stimulation, sham stimulation was compared to each other stimulation. The main interaction and the triple interaction between CCT, CPT and stimulations including all double interactions were analyzed.

For the GLMM of the phases before and after which transcranial stimulation was applied also the categorial fixed factors CCT and stimulation were sum-coded together with time (factor levels: before stimulation, after stimulation). For the fixed effect stimulation, sham stimulation was compared to each other stimulation. We analyzed the main effects of all three factors, all double interactions and their triple interaction. 
We report the Z-values and p-values of the effects via the Welch-Satterthwaite's approximation method (Kuznetsova et al., 2017).

3.3.6.3 Time-frequency analysis Only 23 participants met the inclusion criterion of having at least 20 trials per condition and therefore were included in all analyses. Based on the observation that non-phase locked theta power is predictive of behavioral outcomes (Cohen and Donner, 2013), we excluded theta power phase-locked to stimulus or response in the time-domain from our analysis.

The time-frequency power was calculated from $2 \mathrm{~Hz}$ to $30 \mathrm{~Hz}$ using a 5 cycle Morlet wavelet (gwdith $=2$ ). The complete epoch was baseline normalized into normchange space against the mean value between $-400 \mathrm{~ms}$ to $-100 \mathrm{~ms}$. As first step the averaged time-frequency power from all conditions and participants was plotted per electrode. Three electrodes (FC1, FC2, Cz) with strongest theta power changes over the midfrontal areas were selected for further analysis .

The data of these three electrodes was averaged over all participants, times, trials and conditions. As this grandaverage incorporates all data, it is orthogonal to any possible differences between conditions. The time-frequency window of interest $(400 \mathrm{~ms}-750 \mathrm{~ms}, 3.7 \mathrm{~Hz}-6.6 \mathrm{~Hz})$ is similarly reported in previous literature, and was chosen in accordance with a local maximum in the grandaveraged data (Hanslmayr et al., 2008). This narrow approach best serves to test the hypothesis that the clearly defined cognitive processes during Stroop task were changed by after-effects of transcranial stimulation.

Average over $\mathrm{FC} 1 / \mathrm{FC2} / \mathrm{Cz}$

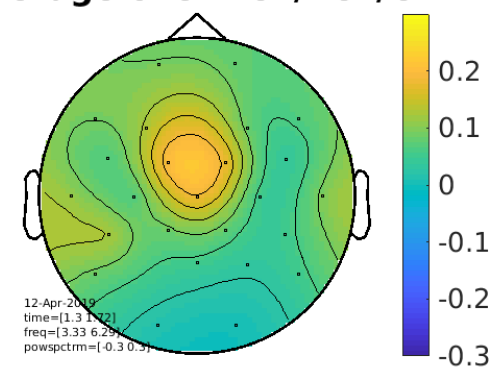

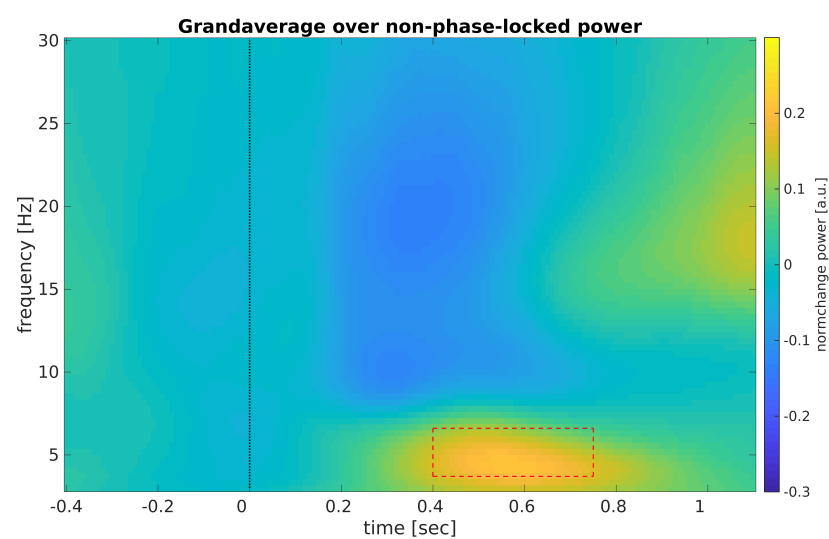

Figure 10: Time-frequency data from the electrodes $\mathrm{FC} 1, \mathrm{FC} 2$ and $\mathrm{Cz}$ was averaged over congruency conditions, time points and participants. The left panel shows the topographical distribution of the local maximum in the ROI. In the right panel the region of interest (ROI, red box) was determined by a local maximum in theta power that was not phase-locked to presentation of stimulus (dotted line). The region of interest is between $400 \mathrm{~ms}$ to $750 \mathrm{~ms}$ and $3.7 \mathrm{~Hz}$ to $6.6 \mathrm{~Hz}$. 
Statistical analysis of EEG The mean power value within this time-frequency window was extracted for every trial. The values were exported to $\mathrm{R}$ and entered into a 2 linear mixed model with three fixed factors (CCT, factor levels: congruent vs. incongruent; time, factor levels: before stimulation, after stimulation; stimulation condition, factor levels: $4 \mathrm{~Hz}$ tACS, $6 \mathrm{~Hz}$ tACS, tRNS, sham). Assumptions of normality were tested both by plotting and by Shapiro-Wilktests. While the tests were more compatible with the time-frequency data not being normally distributed, the visual inspection of histograms and quantile-quantile plots confirmed that the data can be assumed to be distributed normally. We report the Z-values and p-values of the effects via the Welch-Satterthwaite's approximation method (Kuznetsova et al., 2017).

3.3.6.4 Drift diffusion models for conflict tasks We used the drift diffusion model for conflict tasks (DMC) to model the behavioral data (Ulrich et al., 2015). This model allows decomposing RT and accuracy of two-alternative forced choice tasks into several parameters that underlie the decision process. The accumulation of evidence begins with the first information that reaches the brain, and as soon as it reaches a certain threshold for one alternative, a decision is being made (for an introduction please consult (Ratcliff, 1978; Ratcliff and McKoon, 2008). Total RT is divided into the decision process (D) and the residual time (R), which includes the sensory processing of stimulus and response execution (Ulrich et al., 2015). DMC decomposes the D into several parameters by accounting for the RT and accuracy of both congruent and incongruent trials. For a response to occur the boundary (a) has to be crossed by the evidence accumulation. $\mathrm{R}$ is characterized by the nondecision (Ter) and the variability of Ter $\left(\mathrm{s}_{r}\right)$. A controlled process integrates task-relevant information whereas an automatic process integrates task-irrelevant information. The controlled process has a constant drift rate $\left(\mu_{c}\right)$, whereas the drift rate of the automatic process is changing over time. It's best described by a gamma density function which decays over time after an early maximum. The underlying parameters amplitude $(\zeta)$, the time point of maximal amplitude $\left(\mathrm{t}_{\max }\right)$ and the time at which 90 percent of the distribution has decayed ( $\mathrm{t}_{90}$; Ulrich et al., 2015). We fitted the model as has been described by Lehr et al. (2019). 


\subsection{Results}

\subsubsection{Behavioral results}

Error trials were excluded from all RT analyses.

3.4.1.1 Stimulation phase The accuracy was $96.66 \%$ (SD 2.26\%), and the mean RT were $591.83 \mathrm{~ms}$ (SD $65.53 \mathrm{~ms}$ ). The accuracy was higher and RT shorter in congruent trials compared to incongruent trials throughout all stimulation conditions (see Table 7).

Table 7: Descriptive statistics of the stimulation phase. The behavioral difference between incongruent and congruent trials is shown for each stimulation condition. Mean values and standard deviations are reported.

\begin{tabular}{ccc}
\hline & Accuracy $(\%)$ & Response times $(\mathrm{ms})$ \\
\hline Stimulation phase & $96.66 \pm 2.26$ & $591.83 \pm 65.53$ \\
Sham & & \\
Congruent & $97.20 \pm 2.21$ & $577.63 \pm 66.25$ \\
Incongruent & $96.17 \pm 3.09$ & $608.17 \pm 78.85$ \\
6 Hz tACS & & \\
Congruent & $96.89 \pm 2.46$ & $587.00 \pm 67.86$ \\
Incongruent & $96.49 \pm 2.44$ & $607.76 \pm 74.56$ \\
4 Hz tACS & & \\
Congruent & $96.86 \pm 2.91$ & $582.27 \pm 68.92$ \\
Incongruent & $95.95 \pm 3.10$ & $612.21 \pm 82.47$ \\
tRNS & & \\
Congruent & $97.28 \pm 2.17$ & $569.37 \pm 71.80$ \\
Incongruent & $96.40 \pm 2.29$ & $592.09 \pm 77.35$ \\
\hline
\end{tabular}

In Table 8 the estimates of the RT excluding all error trials $(3.33 \%$ of all trials) and the result of the statistical testing are shown. Congruent trials were answered faster than incongruent trials $(\Delta 23.97 \mathrm{~ms})$. All active stimulation conditions changed the RT compared to sham stimulation. While RT were longer for $6 \mathrm{~Hz}(\Delta 4.45 \mathrm{~ms})$ and $4 \mathrm{~Hz}(\Delta 3.33 \mathrm{~ms})$ stimulation, they were shorter for tRNS $(\Delta 12.77)$ stimulation. The Stroop effect was reduced under $6 \mathrm{~Hz}$ stimulation by $8.08 \mathrm{~ms}$ compared to under sham stimulation (Figure 11). tRNS stimulation also reduced the Stroop effect by $5.58 \mathrm{~ms}$ without being significantly different. The conflict adaptation effect $(\mathrm{CCt} \times \mathrm{CPT})$ was also detected in which preceding incongruent trials reduced the Stroop effect by $15.01 \mathrm{~ms}$ compared to preceding congruent trials. There was no interaction between 
stimulation conditions and the conflict adaptation effect. Therefore, the Stroop effect was not preferentially changed in trials that preceded by congruent trials under $6 \mathrm{~Hz}$ tACS but in trials irregardless of the congruency of the preceding trial.

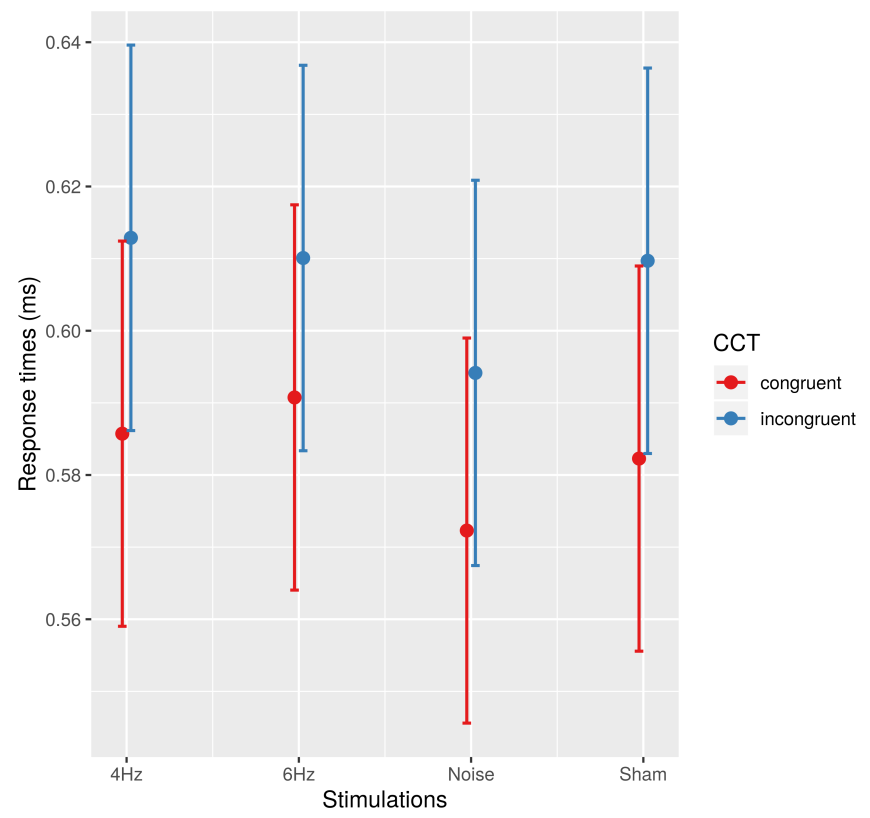

Figure 11: Effect of stimulation on the Stroop effect. For each stimulation condition the response times for incongruent and congruent trials are plotted. The difference between these response times (Stroop effect) is apparent throughout all stimulation conditions. The Stroop effect is reduced for $6 \mathrm{~Hz}$ and tRNS stimulation each compared to sham stimulation. $4 \mathrm{~Hz}$ stimulation has not changed the Stroop effect. All data is plotted including the $90 \%$ confidence interval. CCT, congruency of current trial.

3.4.1.2 Before and after stimulation phase The accuracy was $97.04 \%$ (SD 1.97\%), and the mean RT were $597.98 \mathrm{~ms}$ (SD $65.53 \mathrm{~ms}$ ). The accuracy was higher and RT shorter in congruent trials compared to incongruent trials throughout all stimulation conditions (see Table 9). All nontransformed RT excluding all error trials ( $2.96 \%$ of all trials) were modelled in a GLMM. Table 10 details the results.

Response times were shorter for congruent trials than for incongruent trials ( $\Delta 32.03 \mathrm{~ms}$ ). While RT were longer for $6 \mathrm{~Hz}$ stimulation $(\Delta 2.67 \mathrm{~ms})$, they were shorter for tRNS ( $\Delta 10.63 \mathrm{~ms})$ stim- 
Table 8: The response times in the stimulation phase are reported. CCT, congruency of current trial; CPT, congruency of previous trial.

\begin{tabular}{|c|c|c|c|}
\hline & Response times (ms) & | Z value | & $\mathrm{p}$ value \\
\hline & Estimate (mean $\pm \mathrm{SE})$ & & \\
\hline CCT (Stroop effect) & $\Delta 23.97$ & 26.23 & $<0.001$ \\
\hline Congruent & $582.76 \pm 16.20$ & & \\
\hline Incongruent & $606.73 \pm 16.20$ & & \\
\hline \multicolumn{4}{|l|}{ Stimulation } \\
\hline Sham & $596.00 \pm 16.22$ & & \\
\hline $6 \mathrm{~Hz}$ (vs. sham) & $\Delta 4.45$ & 5.87 & $<0.001$ \\
\hline $6 \mathrm{~Hz}$ & $600.44 \pm 16.22$ & & \\
\hline $4 \mathrm{~Hz}$ (vs. sham) & $\Delta 3.33$ & 4.73 & $<0.001$ \\
\hline $4 \mathrm{~Hz}$ & $599.32 \pm 16.22$ & & \\
\hline tRNS (vs. sham) & $\Delta 12.77$ & 12.27 & $<0.001$ \\
\hline tRNS & $583.22 \pm 16.21$ & & \\
\hline \multicolumn{4}{|l|}{ CCT $\mathrm{x}$ stimulation } \\
\hline Stroop effect (sham) & $27.44 \pm 16.25$ & & \\
\hline Stroop effect (6 Hz vs. sham) & $\Delta 8.08$ & 2.38 & 0.02 \\
\hline Stroop effect $(6 \mathrm{~Hz})$ & $19.37 \pm 16.26$ & & \\
\hline Stroop effect (4 Hz vs. sham) & $\Delta 0.25$ & 1.67 & 0.09 \\
\hline Stroop effect $(4 \mathrm{~Hz})$ & $27.19 \pm 16.26$ & & \\
\hline Stroop effect (tRNS vs. sham) & $\Delta 5.58$ & 1.12 & 0.26 \\
\hline Stroop effect (tRNS) & $21.86 \pm 16.25$ & & \\
\hline $\mathrm{CCT} \times \mathrm{CPT}$ & $\Delta 15.01$ & 6.79 & $<0.001$ \\
\hline Stroop effect (CPT: congruent) & $31.47 \pm 16.22$ & & \\
\hline Stroop effect (CPT: incongruent) & $16.46 \pm 16.22$ & & \\
\hline \multicolumn{4}{|l|}{ CCT x CPT x stimulation } \\
\hline CCT x CPT (6 Hz vs. sham) & $\Delta 0.70$ & 0.26 & 0.80 \\
\hline CCT x CPT (4 Hz vs. sham) & $\Delta 0.53$ & 0.06 & 0.95 \\
\hline CCT x CPT (tRNS vs. sham) & $\Delta 1.35$ & 0.28 & 0.78 \\
\hline
\end{tabular}

SE: Standard error. 
Table 9: Descriptive statistics of the pre- and post- stimulation phases combined. The behavioral difference between incongruent and congruent trials is shown for each stimulation condition. Mean values and standard deviations are reported.

\begin{tabular}{ccc}
\hline & Accuracy $(\%)$ & Response times $(\mathrm{ms})$ \\
\hline Pre/post-stimulation & $97.04 \pm 1.97$ & $597.98 \pm 65.53$ \\
Sham & & \\
Congruent & $97.45 \pm 2.45$ & $582.23 \pm 67.72$ \\
Incongruent & $96.67 \pm 2.73$ & $618.48 \pm 78.02$ \\
6 Hz tACS & & \\
Congruent & $97.59 \pm 2.65$ & $585.73 \pm 73.34$ \\
Incongruent & $96.28 \pm 3.15$ & $621.41 \pm 83.39$ \\
4 Hz tACS & & \\
Congruent & $97.72 \pm 1.69$ & $581.94 \pm 69.38$ \\
Incongruent & $96.39 \pm 2.79$ & $613.66 \pm 79.75$ \\
tRNS & & \\
Congruent & $98.02 \pm 1.63$ & $574.57 \pm 79.12$ \\
Incongruent & $96.17 \pm 3.04$ & $608.90 \pm 87.04$ \\
\hline
\end{tabular}

ulation. Factor time strongly affected RT. After stimulation participants responded on average $32.03 \mathrm{~ms}$ faster than before stimulation.

The data before and after the stimulation suggests that all three active stimulation conditions did not change the Stroop effect. Only the factor time reduced the Stroop effect by $15.42 \mathrm{~ms}$ comparing after to before stimulation. This interaction between time and Stroop effect was not changed by stimulation.

\subsubsection{EEG results}

The non-phase locked oscillary activity within the region of interest $(400 \mathrm{~ms}-750 \mathrm{~ms}, 3.7 \mathrm{~Hz}$ $-6.6 \mathrm{~Hz}$ ) is reported normchange normalized. A GLMM was fitted to all correctly answered trials that were not included during EEG preprocessing. With the supplementary material 3.6 all results of the statistical analysis are provided (Table 11). 
Table 10: The response times from before and after stimulation are reported. CCT, congruency of current trial.

\begin{tabular}{|c|c|c|c|}
\hline & Response times (ms) & | Z value I & $\mathrm{p}$ value \\
\hline & \multicolumn{3}{|l|}{ Estimate (mean $\pm \mathrm{SE}$ ) } \\
\hline CCT (Stroop effect) & $\Delta 32.03$ & 26.31 & $<0.001$ \\
\hline Congruent & $586.81 \pm 17.61$ & & \\
\hline Incongruent & $618.85 \pm 17.61$ & & \\
\hline Time & $\Delta 32.03$ & 14.37 & $<0.001$ \\
\hline before stimulation & $618.85 \pm 17.61$ & & \\
\hline after stimulation & $586.81 \pm 17.61$ & & \\
\hline \multicolumn{4}{|l|}{ Stimulation } \\
\hline Sham & $605.39 \pm 17.63$ & & \\
\hline $6 \mathrm{~Hz}$ (vs. sham) & $\Delta 2.67$ & 4.89 & $<0.001$ \\
\hline $6 \mathrm{~Hz}$ & $608.06 \pm 17.63$ & & \\
\hline $4 \mathrm{~Hz}$ (vs. sham) & $\Delta 2.26$ & 0.28 & 0.78 \\
\hline $4 \mathrm{~Hz}$ & $603.12 \pm 17.63$ & & \\
\hline tRNS (vs. sham) & $\Delta 10.63$ & 7.73 & $<0.001$ \\
\hline tRNS & $594.75 \pm 17.63$ & & \\
\hline \multicolumn{4}{|l|}{ CCT $\mathrm{x}$ stimulation } \\
\hline Stroop effect (Sham) & $33.87 \pm 17.67$ & & \\
\hline Stroop effect (6 Hz vs. sham) & $\Delta 1.49$ & 0.16 & 0.87 \\
\hline Stroop effect (6 Hz) & $32.38 \pm 17.67$ & & \\
\hline Stroop effect (4 Hz vs. sham) & $\Delta 3.50$ & 0.79 & 0.43 \\
\hline Stroop effect (4 Hz) & $30.38 \pm 17.67$ & & \\
\hline Stroop effect (tRNS vs. sham) & $\Delta 2.37$ & 0.25 & 0.80 \\
\hline Stroop effect (tRNS) & $30.38 \pm 17.67$ & & \\
\hline CCT x Time & $\Delta 15.42$ & 6.34 & $<0.001$ \\
\hline Stroop effect (Time: before) & $39.74 \pm 17.63$ & & \\
\hline Stroop effect (Time: after) & $24.33 \pm 17.63$ & & \\
\hline \multicolumn{4}{|l|}{ CCT $\mathrm{x}$ Time $\mathrm{x}$ stimulation } \\
\hline CCT x Time (6 Hz vs. sham) & $\Delta 0.83$ & 0.83 & 0.40 \\
\hline CCT x Time (4 Hz vs. sham) & $\Delta 8.06$ & 1.27 & 0.20 \\
\hline CCT x Time (tRNS vs. sham) & $\Delta 3.63$ & 0.22 & 0.82 \\
\hline
\end{tabular}

SE: Standard error. 


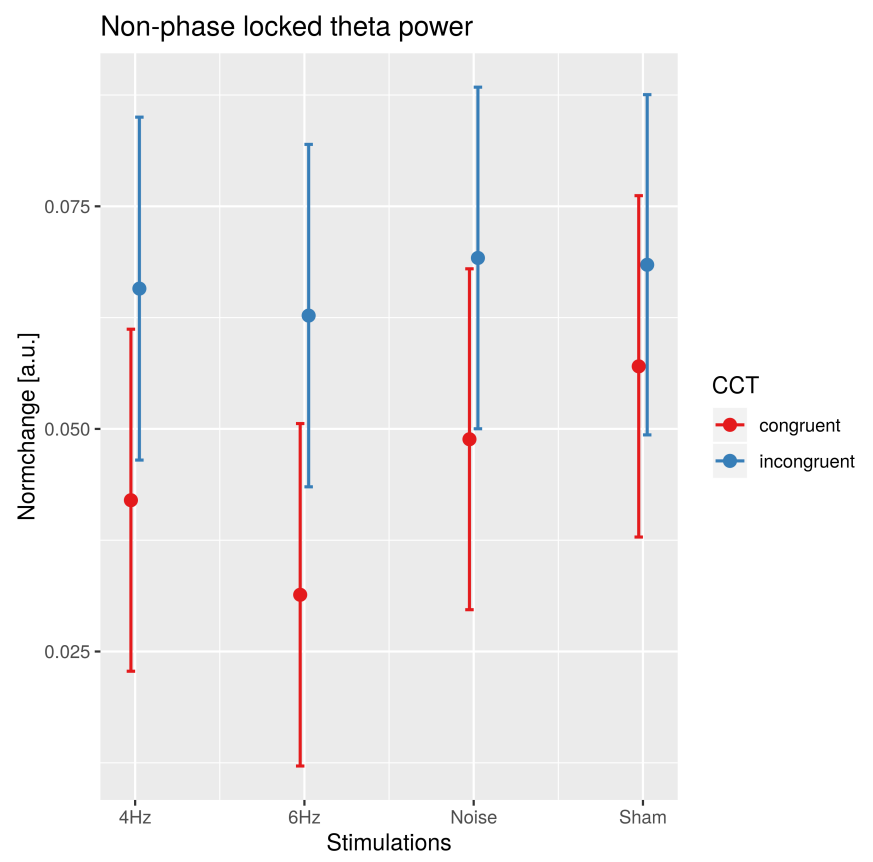

Figure 12: Effect of stimulation on the difference in theta power between congruent and incongruent trials. The difference is apparent throughout all stimulation conditions and resembles a Stroop effect. All active stimulations, but especially $6 \mathrm{~Hz}$ stimulation, show an increased difference compared to sham stimulation. All data is plotted including the $90 \%$ confidence interval. CCT, congruency of current trial.

Non-phase locked theta power increased in incongruent trials compared to congruent trials. This is the well-described neural equivalent of the Stroop effect. Oppositely, theta power was reduced within in phase after stimulation compared to before stimulation. $6 \mathrm{~Hz}$ stimulation reduced theta power compared to sham over all trials. The data shows a trend that $6 \mathrm{~Hz}$ stimulation also interacted with the equivalent of the Stroop effect, resulting in an increased difference in incongruent and congruent trials' theta power (Figure 12). The interaction between the factor Time and the equivalent of the Stroop effect was unaffected by $6 \mathrm{~Hz}$ stimulation. Similarly, no main or interaction effects of the other active stimulations occured. 

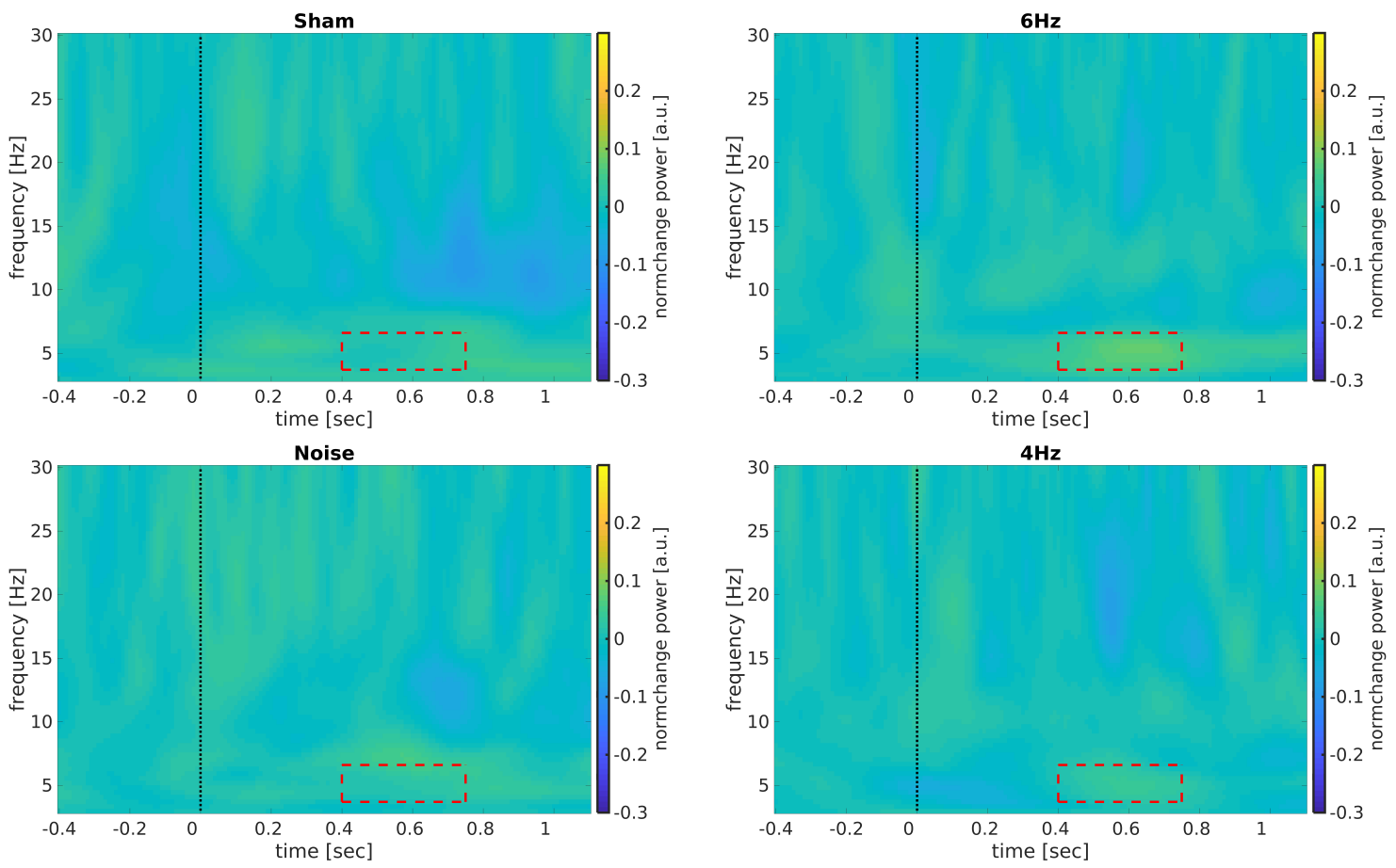

Figure 13: The difference in non-phase locked theta power between incongruent and congruent trials after stimulation is plotted for every stimulation. While the difference is increased within the ROI (400 $\mathrm{ms}$ to $750 \mathrm{~ms}$ and 3.7 Hz to $6.6 \mathrm{~Hz}$ ) for $6 \mathrm{~Hz}$ stimulation compared to sham stimulation, no statistical significance was observed. The other stimulations did not change the difference compared to sham stimulation.

\subsubsection{Drift diffusion model for conflict tasks}

The mean $\mathrm{G}^{2}$ score of the fitted DMC for all sessions was 14.11 (SD 16.12). For statistically testing the DMC parameters, we employed a Monte Carlo approximative Kruskal-Wallis test and corrected for multiple comparison via the Holm-Bonferroni method. Post-hoc tests were not performed as no statistically significant differences were detected: a $(\operatorname{maxT}=0.58, \mathrm{p}=1)$, $\left.\mu_{c}(\max T=0.72, \mathrm{p}=1), \mathrm{p}=1\right), \zeta(\max \mathrm{T}=2.89, \mathrm{p}=0.11), \mathrm{t}_{90}(\operatorname{maxT}=1.49, \mathrm{p}=1)$, and $\mathrm{t}_{\max }$ $(\max \mathrm{T}=1.66, \mathrm{p}=1) \operatorname{Ter}(\max \mathrm{T}=0.56, \mathrm{p}=1), \mathrm{s}_{t}(\max \mathrm{T}=0.96, \mathrm{p}=1)$.

\subsection{Discussion}

Midfrontal theta oscillations correlate with cognitive control (Hanslmayr et al., 2008; Cohen and Donner, 2013; Cavanagh and Frank, 2014), but causality has been difficult to demonstrate. Identification of the causal mechanisms behind cognitive control may elucidate how brain processes are integrated to control behavior. 
We applied midfrontal tACS and tRNS to healthy volunteers performing a Stroop task. First, only tACS, specifically in the mid-theta range, reduced the Stroop effect as hypothesized. $6 \mathrm{~Hz}$ tACS reduced the Stroop effect during stimulation. $4 \mathrm{~Hz}$ tACS, which acted as a slightly out-ofrange active control, did not reduce the Stroop effect. Second, we expected reduction in Stroop effect to be driven predominantly by a strong reduction in trials preceded by congruent trials due comparatively low activation of the $\mathrm{CCN}$. As this was not the case, the effect reduction in Stroop effect happened irregardless of the congruency of the previous trial.

A study describing a similar stimulation in a Simon task found also that the conflict effect was reduced by tACS in mid-theta rage, but here driven by a reduction in trial preceded by congruent trials (van Driel et al., 2015). To et al. (2018) showed for a similar electrode montage as used in this study that transcranial direct current stimulation influenced both RT during and theta-band EEG activity after a cognitive and emotional Counting Stroop task. Reductions in conflict effects after theta-range stimulation to the DLPFC occurred are reported for Stroop task and Balloon analog risk task (Sela et al., 2012; Lehr et al., 2019). The main results of this study are in line with previous research and give further evidence that the midfrontal neural oscillator dACC that emits theta range oscillations cause the Stroop effect.

However, the influence of $6 \mathrm{~Hz}$ stimulation on the Stroop effect did not persist into the phase after stimulation. While induced changes in brain physiology have been described to persist up to 70 minutes (Kasten et al., 2016), Heise et al. (2019) acknowledge that effects of tACS on task performance might differ during and after stimulation. While the absence of effect after stimulation does not invalidate the behavioral effects during stimulation, it casts a doubt if tACS-induced physiological changes in higher brain functions are durable beyond immediate stimulation and impedes interpretation of the EEG results.

Surprinsingly, the EEG time-frequency data showed a trend towards an increased Stroop-like effect after $6 \mathrm{~Hz}$ stimulation. Contrary to our hypothesis, the difference in non-phase locked midline theta power increased between congruent and incongruent trials compared to sham stimulation. As midline theta power has been described to correlate positively with cognitive control and negatively with Stroop effect (Hanslmayr et al., 2008; Oehrn et al., 2014), it remains puzzling that there was no simultaneous increase in the behavioral Stroop effect after stimulation. We can not exclude that the reduction in behavioral Stroop effect during $6 \mathrm{~Hz}$ stimulation was accompanied by similar theta power changes as found after stimulation. This would demote 
the proposed mechanism that increased theta power especially in congruent trials causes the reduction in behavioral Stroop effect.

tRNS reduced the average RT by around $10 \mathrm{~ms}$ in the data acquired simultaneously to stimulation. A similar decrease in average RT occurred in the data acquired before and after stimulation. TRNS was reported to induce behavioral changes without RT differences (Cappelletti et al., 2013). Upon further inspection of the data, the RT for tRNS were reduced compared to sham stimulation even before the stimulation phase and are therefore most likely an artifact unrelated to the independent variables. We also confirm that practice (reported as factor time) decreased RT generally and interacted with CCT to reduce Stroop effect (Davidson et al., 2003). We hypothesized that the DMC would reflect the decreased Stroop effect with a weakened influence of the automatic process (word reading). However, contrary to our hypothesis no parameter governing the automatic function $\left(\zeta, \mathrm{t}_{90}, \mathrm{t}_{\max }\right)$ reflected that. The statistical power of the DMC was insufficient for detecting that the Stroop effect was reduced during $6 \mathrm{~Hz}$ stimulation by $29.4 \%$ compared to during sham stimulation.

One of the limitations of this study is that EEG was not measured concurrently to transcranial stimulation due to stimulation artifacts in the EEG data (Helfrich et al., 2014; Kohli and Casson, 2019, 2020). The behavioral changes during stimulation match expectations, but the trend in the EEG data are actually opposite of what was expected and are not corroborated by simultaneous changes in behavior. The question if the dACC-DLPFC network was entrained by $6 \mathrm{~Hz}$ stimulation and if the theta power difference was reduced during $6 \mathrm{~Hz}$ stimulation remains elusive.

Non-phase locked theta power is sufficiently described in the literature (Hanslmayr et al., 2008; Nigbur et al., 2011; Cohen and Donner, 2013; Oehrn et al., 2014) and lends itself for hypothesis testing, but more fine-grained source-based analyses might have allowed more exploratory insights. Time-frequency analyses have two main drawbacks: Low temporal resolution, especially in lower frequency bands, and sheer amount of possible analyses (Cohen, 2014b). However, we specifically analyzed the well-described increased theta connectivity between dACC and DLPFC that occurs from $250 \mathrm{~ms}$ to $600 \mathrm{~ms}$ after stimulus presentation (Hanslmayr et al., 2008; Oehrn et al., 2014). The here-used hypothesis-driven analysis of time-frequency data massively shrinks the space of possible analyses and forecludes statistical multiple-comparison problems (Cohen, 2014b).

In the future the stimulation of DLPFC and dACC simulteanously by mid-theta tACS but with 
3 THETA TRANSCRANIAL ALTERNATING CURRENT STIMULATION DECREASES CONFLICT EFFECT IN STROOP TASK

differing phases of stimulation could yield insights into phase-specificity of their interaction. With the dACC hypothesized to lead the interaction during conflict and the DLPFC after conflict resolution (for adaptation; Oehrn et al. (2014)), the stimulation could be either in parallel with conflict or with adaptation after conflict. Based on their roles, stimulation with a phase-lead of dACC to DLPFC should improve conflict resolution but decrease adaptation in the next trials.

This is the first study exploring the causal role of theta power during Stroop task. Stimulation with theta tACS proves the dACC to be central to producing the Stroop effect. The key questions to be answered are (a) how theta power changes simultaneously to stimulation and (b) if the communication between dACC and DLPFC is susceptible to phase-shifted stimulation. 


\subsection{Supplementary material}

Table 11: The non-phase locked oscillatory power before and after stimulation was normchange normalized [a.u.]. CCT, congruency of current trial; CPT.

\begin{tabular}{|c|c|c|c|}
\hline & Theta power [normchange - a.u.] & | Z value | & $\mathrm{p}$ value \\
\hline & \multicolumn{3}{|l|}{ Estimate (mean $\pm \mathrm{SE})$} \\
\hline CCT (Stroop effect) & $\Delta 0.02$ & 5.19 & $<0.001$ \\
\hline Congruent & $0.04 \pm 0.01$ & & \\
\hline Incongruent & $0.07 \pm 0.01$ & & \\
\hline Time & $\Delta 0.02$ & 10.20 & $<0.001$ \\
\hline before stimulation & $0.07 \pm 0.01$ & & \\
\hline after stimulation & $0.04 \pm 0.01$ & & \\
\hline \multicolumn{4}{|l|}{ Stimulation } \\
\hline Sham & $0.06 \pm 0.01$ & & \\
\hline $6 \mathrm{~Hz}$ (vs. sham) & $\Delta 0.02$ & 2.28 & 0.02 \\
\hline $6 \mathrm{~Hz}$ & $0.05 \pm 0.01$ & & \\
\hline $4 \mathrm{~Hz}$ (vs. sham) & $\Delta 0.01$ & 0.42 & 0.67 \\
\hline $4 \mathrm{~Hz}$ & $0.05 \pm 0.01$ & & \\
\hline tRNS (vs. sham) & $\Delta 0.00$ & 0.76 & 0.45 \\
\hline tRNS & $0.06 \pm 0.01$ & & \\
\hline \multicolumn{4}{|l|}{ CCT $\mathrm{x}$ stimulation } \\
\hline Stroop effect (Sham) & $0.01 \pm 0.01$ & & \\
\hline Stroop effect (6 Hz vs. sham) & $\Delta 0.02$ & 1.33 & 0.18 \\
\hline Stroop effect $(6 \mathrm{~Hz})$ & $0.03 \pm 0.01$ & & \\
\hline Stroop effect (4 Hz vs. sham) & $\Delta 0.01$ & 0.25 & 0.80 \\
\hline Stroop effect (4 Hz) & $0.02 \pm 0.01$ & & \\
\hline Stroop effect (tRNS vs. sham) & $\Delta 0.01$ & 0.14 & 0.89 \\
\hline Stroop effect (tRNS) & $0.02 \pm 0.01$ & & \\
\hline CCT x Time & $\Delta 0.00$ & 0.04 & 0.96 \\
\hline Stroop effect (Time: before) & $0.02 \pm 0.01$ & & \\
\hline Stroop effect (Time: after) & $0.02 \pm 0.01$ & & \\
\hline \multicolumn{4}{|l|}{ CCT $\mathrm{x}$ Time $\mathrm{x}$ stimulation } \\
\hline CCT x Time (6 Hz vs. sham) & $\Delta 0.02$ & 0.33 & 0.74 \\
\hline CCT x Time (4 Hz vs. sham) & $\Delta 0.01$ & 0.24 & 0.81 \\
\hline CCT x Time (tRNS vs. sham) & $\Delta 0.03$ & 0.81 & 0.42 \\
\hline
\end{tabular}

SE: Standard error. 


\section{Theta-gamma cross-frequency transcranial alternating cur- rent stimulation over the trough impairs cognitive control}

Authors: Zsolt Turi ${ }^{1 *}$, Matthias Mittner ${ }^{2 *}$, Albert Lehr ${ }^{1}$, Hannah Bürger ${ }^{1}$, Andrea Antal ${ }^{1}$, and Walter Paulus ${ }^{1}$

*Equal contribution

Affiliations: ${ }^{1}$ Department of Clinical Neurophysiology, University Medical Center Göttingen, Göttingen 37073, Germany

${ }^{2}$ UiT The Arctic University of Norway

eNeuro, submitted

psyarxiv: doi: 10.31234/osf.io/4y3uh

Author contributions:

ZT: conceptualization, study design, project administration, methodology, software (behavioral paradigm), supervised data collection, supervised medical student, prepared illustrations, data visualization, interpreted data, data curation and wrote original draft.

MM: formal statistical analysis, data visualization, computational modelling of behavioral data, interpreted data, data curation and wrote original draft.

Detailed author contribution of AL: contributed to formal analysis, contributed to preparing illustrations, data visualization, interpreted data and wrote original draft.

HB: data collection (as part of her medical dissertation at the University Medical Center Goettingen, Germany, supervised by author AA), transcribed data, contributed to writing original draft. The medical dissertation of HB is based on this data.

AA: project administration, supervised medical student, contributed to writing original draft.

WP: study design, resources and funding acquisition, contributed to writing original draft.

The citation style of the manuscript has been changed to match the citation style of the dissertation. Its bibliography is included in the general bibliography of the dissertation.

Copyright (C) 2020 Zsolt Turi et al. CC-By Attribution 4.0 International 


\subsection{Abstract}

Cognitive control is a hypothetical mental process, which underlies adaptive goal-directed decisions. Previous studies have linked cognitive control to electrophysiological fluctuations in the theta band and theta-gamma cross-frequency coupling (CFC) arising from the cingulate and frontal cortices. Yet, to date the behavioral consequences of different forms of theta-gamma CFC remain elusive. Here, we studied the behavioral effects of the theta-gamma CFC via transcranial alternating current stimulation (tACS) designed to stimulate the frontal and cingulate cortices. Using a double-blind, randomized, repeated measures study design, 24 healthy participants were subjected to three main, active CFC-tACS protocols: Short gamma frequency bursts $(80 \mathrm{~Hz})$ were coupled to an ongoing theta cycle $(4 \mathrm{~Hz})$ to coincide with either the peaks or the troughs of the theta wave. In a third condition, the amplitude of the gamma oscillation was modulated by the phase of a theta cycle. In the fourth, control protocol, gamma was continuously superimposed over the theta cycle, therefore lacking any phase-specificity in the CFC. During the 20-minute stimulations, the participants performed a Go/NoGo monetary reward- and punishment-based instrumental learning task. A Bayesian hierarchical logistic regression analysis revealed that CFC-tACS over peak had no effects on the behavioral performance, whereas CFC-tACS over trough and, to a lesser extent, amplitude-modulated tACS reduced performance in conflicting trials. Our results suggest that cognitive control depends on the phase-specificity of the theta-gamma CFC.

\subsection{Introduction}

Goal-directed behavior requires the organism to effectively link contextual and reward-related information to action plans in order to accomplish goals (Redish, 2016). In humans, at least three main behavioral control systems can influence goal-directed behavior: The Pavlovian system, the model-free and the model-based systems (Guitart-Masip et al., 2014). The Pavlovian system is responsible for automatic, reflexive response tendencies that depend on the learned valence of the stimulus. This behavioral control system facilitates approaching behavior for rewarding stimuli and response inhibition for unrewarding ones (Guitart-Masip et al., 2014). However, conflict can arise when these evolutionary hard-wired, valence-response associations do not support adaptive behavior (Cavanagh et al., 2013). This situation occurs when approach- 
ing rewards is maladaptive, or when rewards can be secured by response inhibition rather than by approach (Guitart-Masip et al., 2012). Cognitive control is a hypothetical, effortful mental process for resolving this conflict between the behavioral control systems (Guitart-Masip et al., 2014; Shenhav et al., 2017).

It has been proposed that the oscillatory activity in the theta and gamma frequency bands and their interaction play a crucial role in cognitive control (Amemiya and Redish, 2018; Cavanagh and Frank, 2014; Cohen, 2014a). Theta-gamma, phase-amplitude cross-frequency coupling is one form of such interaction, where the phase of the theta oscillation modulates the amplitude of the gamma oscillation (Canolty and Knight, 2010). Human intracranial electrophysiological recordings revealed that theta-gamma, phase-amplitude cross-frequency coupling in the anterior cingulate cortex (ACC) and dorsolateral prefrontal cortex (DLPFC) emerges during cognitive control (Smith et al., 2015). In a Stroop-like cognitive interference task, Smith et al. (2015) found that the amplitude of the high gamma oscillation $(70 \mathrm{~Hz}-125 \mathrm{~Hz})$ was highest in a specific phase range of the theta oscillation (ca. $0^{\circ}-60^{\circ}$ ) during cognitive control (Smith et al., 2015). Information transfer analysis (Granger causality) also showed that the feedback-related information travels from the ACC to the DLPFC in the theta band (Smith et al., 2015). A similar theta-gamma coupling pattern was found in the rat hippocampus during a spatial decisionmaking task (Amemiya and Redish, 2018), suggesting that theta-gamma coupling might be a general neural coding scheme employed by subcortical and cortical structures in the mammalian brain. These findings may suggest that the ACC presumably signals the need for cognitive control, whereas the DLPFC processes this information and influences ongoing behavior by exerting model-based behavioral control (Smith et al., 2015).

To study how participants learn to overcome the automatic Pavlovian bias by utilizing cognitive control mechanisms, we used a probabilistic Go/NoGo monetary reward- and punishmentbased instrumental learning task (Cavanagh et al., 2013). We tested the behavioral relevance of theta-gamma cross-frequency coupling in humans via transcranial alternating current stimulation (tACS), which can externally generate oscillating electric fields in the brain (Antal et al., 2017). We utilized three CFC-tACS protocols: Theta-gamma CFC-tACS over the peak, over the trough, and amplitude-modulated tACS (Alekseichuk et al., 2016; Minami and Amano, 2017; de Lara et al., 2018). In the amplitude-modulated protocol, the amplitude of the gamma oscillation was modulated by the phase of the theta wave. 
We hypothesized that the theta-gamma CFC-tACS over the peak protocol would be beneficial for behavioral performance, i.e., they would improve the accuracy and/or the speed of learning relative to the control condition. We based this hypothesis on the notion that these protocols mimic the phase-specificity of theta-gamma CFC when signaling the need for cognitive control (Smith et al., 2015). Moreover, we also anticipated that theta-gamma CFC-tACS over the trough would impair behavioral performance because this pattern is contrary to that activity naturally occurring during the successful implementation of cognitive control (Smith et al., 2015). Third, we expected that modulating the CFC between the ACC and DLPFC via CFC-tACS protocols should affect the amount of Pavlovian bias. In particular, facilitating the CFC between the ACC and DLPFC via CFC-tACS over the peak would be thought to increase the efficacy of the ACC to signal the need for cognitive control and thereby increase the degree of modelbased control implemented by the DLPFC (Smith et al., 2015). This, in turn, might lead to a decreased amount of Pavlovian bias. On the other hand, disrupting the CFC between the ACC and the DLPFC via CFC-tACS over the trough should decrease the efficacy of signaling the need for cognitive control. This may impair the efficacy of implementing model-based control and therefore lead to a higher degree of Pavlovian bias. Although the physiological effects of the amplitude-modulated tACS are still not completely understood, we expected that it would improve behavioral performance by entraining the ongoing theta oscillation by the envelope of the high frequency stimulation (Negahbani et al., 2018). Similar to single-frequency theta tACS, the amplitude-modulated tACS protocol would increase the theta synchrony in the cingulate and frontal cortices (Negahbani et al., 2018), which in turn would improve the ability of the participants to apply cognitive control.

\subsection{Methods}

\subsubsection{Participants}

Twenty-four healthy, native German-speaking adult volunteers (12 female, mean age \pm SD: $23.0 \pm 3.26$ years, age from 18 to 30 years) joined the study. This number of participants was chosen to allow a complete randomization of the order of the four tACS protocols (i.e., three active and one control protocols), and is calculated as four factorial or 24. The mean number of years of education $( \pm \mathrm{SD}$ ) was $16.30 \pm 3.05$ (range from 12 to 22.5 years). Before enter- 
ing the study, the participants were informed about possible adverse effects of tACS, and all of them gave their written informed consent. The exclusion criteria were history or presence of current medical, neurological or psychiatric illnesses including epilepsy, drug and/or alcohol addiction and the presence of metal implants in the head, neck and chest. In addition, the participants were examined by neurologists at the Department of Clinical Neurophysiology, University Medical Center Göttingen. The study neurologist evaluated whether any of the exclusion criteria were met. None of the participants reported any neurological or psychiatric disorders, drug-dependency, or medication acting on the central nervous system prior to or during the experiment.

\subsubsection{Ethic statement and data availability}

The Ethics Committee of the University Medical Center Göttingen approved the study, the study protocols, and all methods used therein. We performed the study in accordance with relevant guidelines and regulations. The study was registered under the study approval number 20/5/15. All materials, raw data and analysis scripts are available at our repository: https://github.com/ihrke/2020_cfc_tacs.

\subsubsection{Experimental design}

The study used a double-blind, within-subject design. The participants underwent five experimental sessions, starting with an initial training session to familiarize themselves with the behavioral paradigm. During the training session, the participants received no stimulation. This initial session was followed by the four tACS sessions, the order of which was counterbalanced across participants to reduce between-session learning effects. Of the four stimulation sessions, three employed the main stimulation protocols and one the control protocol. The inter-session interval between the stimulation sessions was at least 48 hours.

\subsubsection{Behavioral Paradigm}

The behavioral paradigm consisted of a learning phase and a subsequent transfer phase, which was adapted from Cavanagh et al. (2013). The task was introduced as a card game for the participants (Figure 14). Stimuli presentation was controlled by PsychoPy (version number: 1.83.01), 
a free, open-source application built on the Python programming language Peirce $(2007,2009)$. For the presentation of the behavioral paradigm, we used a Dell computer with Windows 7 Enterprise 64 bit operating system, Intel (R) core i3-3220, 3.30 GHz and 4 GB RAM.

During the learning phase the participants performed a Go/NoGo instrumental learning task. Here, they had to learn action (two levels: Go/NoGo) and monetary outcome (three levels: win, no win/lose or lose) contingencies. For each card, the goal was to find the better of the two possible action choices (Go/NoGo) resulting in the highest monetary outcome (getting reward or avoiding losing) and therefore maximize their earnings.

One key feature of the task was that the action choices and monetary outcomes were orthogonal. As such, the four unique cards covered all the combinations between actions choices and monetary outcomes ('Go to win', 'NoGo to avoid losing', 'Go to avoid losing' and 'NoGo to win'). Due to the Pavlovian bias, i.e., approach to appetitive and withdrawal from aversive stimuli, the cards could be split into congruent and conflicting cards. For the Pavlovian congruent cards (henceforth the congruent trials; 'Go to win' and 'NoGo to avoid losing') the action selection under the automatic, Pavlovian bias was advantageous for the participants and hence easier to learn. For the Pavlovian conflicting cards (henceforth the conflicting trials; 'NoGo to win' and 'Go to avoid losing'), the action selection under the automatic, Pavlovian bias was disadvantageous for the participants and therefore harder to learn (Guitart-Masip et al., 2012).

The action outcomes were probabilistic such that $65 \%$ of correct responses led to a better outcome: Neutral monetary outcomes (no loss) for the lose cards and monetary reward for the win cards. Consequently, 35\% of the correct responses led to neutral monetary outcomes for the win cards and monetary loss for the lose cards. On the other hand, wrong responses inverted this ratio, i.e. $65 \%$ of incorrect responses led to neutral monetary outcomes for the win cards and monetary loss for the lose cards. Previous studies used $80 \%$ vs. $20 \%$ or $70 \%$ vs. $30 \%$ action-outcome contingencies, which renders the present version of the probabilistic learning task slightly more difficult compared with previous versions (Cavanagh et al., 2013; Csifcsák et al., 2020; Guitart-Masip et al., 2012).

Each card was presented 20 times in a random order. Independent sets of four cards were used and randomly chosen for each session. At the beginning of each trial, a fixation cross (10 or $11 \mathrm{~s}$ ) and then a card cue (1 s) were presented to the participants (see Figure 14A, Trial flow). Note that we used a relatively long fixation cross in the present study compared with previous studies 
(e.g., Guitart-Masip et al. (2012)). This was a necessary step to increase the comparability of the present results with another experiment using pre-stimulus intermittent tACS (manuscript in preparation). The target detection stimulus (black circle) was shown until a response occurred, or $1 \mathrm{~s}$ passed. The target detection stimulus indicated to the participants that they could take the card (Go) or not (NoGo), upon which the monetary outcome depended. The feedback was displayed for $2 \mathrm{~s}$ : A green ' $+€$ ' sign indicated a monetary reward, a red '-€' symbol indicated a monetary loss and a black horizontal bar indicated neutral monetary outcome (neither win nor loss). The next trial started $0.5 \mathrm{~s}$ after feedback.

In the subsequent transfer phase of the task (Figure 14B), the participants performed a twoalternative, forced-choice task where each card from the learning phase was paired with one of the three other cards following the order (e.g., 'Go to win' vs. 'NoGo to avoid losing', 'NoGo to avoid losing' vs. 'Go to win', etc.). Each of the 12 card pairs was presented four times. The participants had to select the 'better' card relying on implicit and explicit learning.

The dependent variable in this study was accuracy. We defined accuracy as choosing the response category (Go/NoGo) that led with a higher probability to the better monetary outcome; hence, monetary reward for the win cards and neutral monetary outcome for the losing cards. The participants were paid 8 EUR/hour and received an additional performance dependent bonus of 12 EUR if their mean performance calculated over all sessions was above $75 \%$. We used the monetary bonus to encourage our participants to perform as well as possible in each session. Unknown to the participants, everybody received the monetary bonus at the end of the experiment. 
4 THETA-GAMMA CROSS-FREQUENCY TRANSCRANIAL ALTERNATING CURRENT STIMULATION OVER THE TROUGH IMPAIRS COGNITIVE CONTROL

A Learning phase

Pavlovian congruent
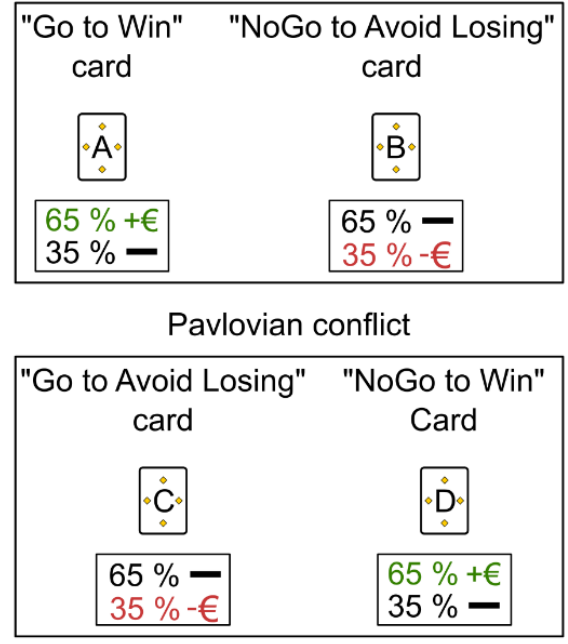

Trial flow

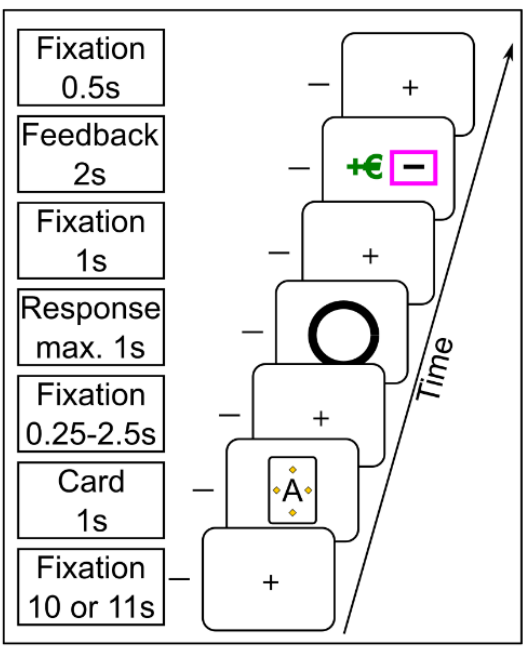

B Transfer phase

All card combinations

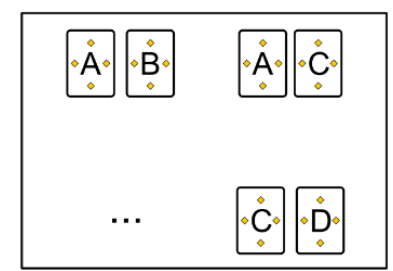

Trial flow

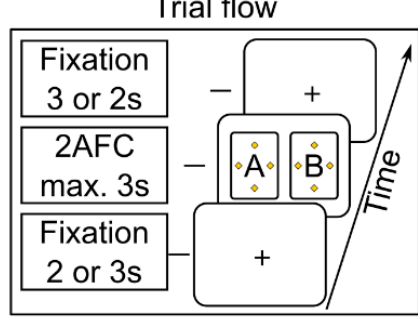

Figure 14: The structure and the trial flow of the behavioral paradigm for the learning (A) and the transfer phase (B).

\subsubsection{Transcranial alternating current stimulation}

The stimulation was delivered by a CE-certified NeuroConn ${ }^{\circledR}$ multichannel stimulator (neuroConn GmbH, Ilmenau, Germany) during the learning phase of the task. The electrode positions were chosen according to the international 10-20 EEG system. The electrode montage was centered over the Fpz electrode location with three return electrodes positioned over the Cz, F10 and F9 positions (Figure 15A). 
A Electrode montage

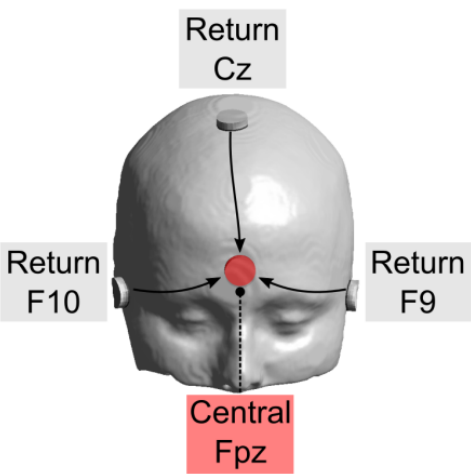

\section{Estimated induced electric field magnitudes}

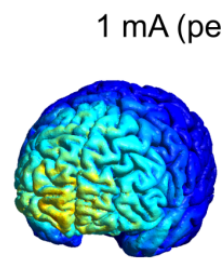

Frontal view

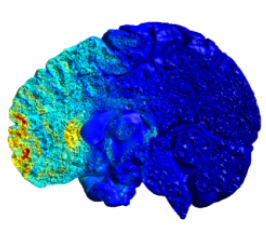

Saggital view
B Waveforms of CFC-tACS protocols

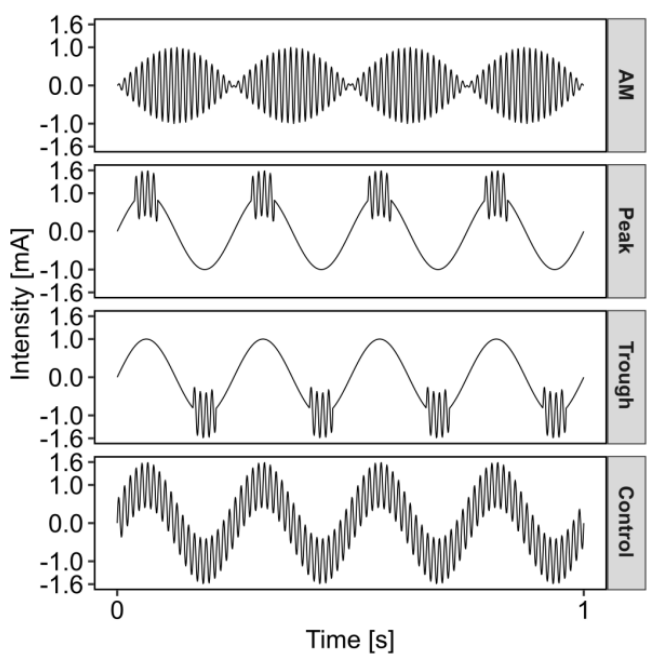

Figure 15: Stimulation parameters including electrode montage (A), cross-frequency-coupling tACS waveforms (B) and estimated electric field magnitudes in the gray matter. Electric field simulations were performed with SimNIBS version 3.0.2. Abbreviations: AM - amplitude-modulated; CFC - cross-frequency coupling.

The following standardized steps ensured minimal stimulation-induced cutaneous sensations. After determining the electrode locations, the corresponding skin surface was gently cleaned with OneStep® abrasive gel (H + H Medizinprodukte GbR, Germany), which was removed with 0.9\% saline solution (B. Braun Melsungen AG, Germany). After removing the residual saline solution with paper tissue a local anesthetic cream (Anesderm®, Pierre Fabre Dermo-Kosmetic $\mathrm{GmbH}$, Germany) was applied for $20 \mathrm{~min}$ to numb the skin $(25 \mathrm{mg} / \mathrm{g}$ lidocaine, $25 \mathrm{mg} / \mathrm{g}$ prilocaine). It was wiped off first with paper tissue followed by a skin antiseptic spray (Kodan Tinktur Forte ${ }^{\circledR}$, Schuelke \& Mayr GmbH, Germany). The latter was necessary to remove the anesthetic cream, which would otherwise prevent the conductive paste from adhering to the skin. Homogenous layers of Ten20® conductive paste (Waever and Company, Colorado, USA) were then applied to the skin and the electrode surfaces. Each of the four round, conductive rubber electrodes with $2 \mathrm{~cm}$ diameter (neuroConn $\mathrm{GmbH}$, Germany) was affixed to the head. The 
impedance was kept below $10 \mathrm{k} \Omega$. The maximal current density under the main electrode was $0.50 \mathrm{~mA} / \mathrm{cm}^{2}$. The electrode montage was prepared in a double-blind fashion.

We used four different CFC-tACS protocols, including amplitude-modulated CFC-tACS (AM), CFC over the peak, trough and control tACS (Figure 15B). Each protocol started with a 20s fade-in period, followed by a 20-min stimulation with the maximum stimulation intensity, and ended with a $10 \mathrm{~s}$ fade-out period. The total stimulation duration was 20 min and $30 \mathrm{~s}$.

The protocols, CFC-tACS over the peak, over the trough, and control, consisted of a $4 \mathrm{~Hz}, 1 \mathrm{~mA}$ ( $=2 \mathrm{~mA}$ peak to peak) sinusoidal waveform coupled with a $0.6 \mathrm{~mA}(=1.2 \mathrm{~mA}$ peak to peak $) 80 \mathrm{~Hz}$ sinusoidal waveform. These stimulation protocols had a maximum intensity of $1.6 \mathrm{~mA}$. In the CFC-tACS peak protocol, the short $80 \mathrm{~Hz}$ burst $(50 \mathrm{~ms})$ was coupled over the peak (38-88 $\mathrm{ms})$ of each theta cycle. In the CFC-tACS trough protocol, the short $80 \mathrm{~Hz}$ burst was coupled over the trough (163-213 ms) of each theta cycle. In the control stimulation, both waveforms were overlaid continuously. The control condition lacked any phase-specificity of gamma relative to theta oscillations but used a highly matched intensity range and identical stimulation duration with respect to the real protocols. The control protocol served as the reference to which we compared the effects of the three main CFC-tACS protocols.

In the AM protocol, the amplitude of the gamma frequency $(80 \mathrm{~Hz})$ was modulated by the phase of the theta frequency $(4 \mathrm{~Hz})$. In all protocols, the amplitude of the theta frequency was constant. Consequently, the AM protocol employed lower peak stimulation intensities compared with the remaining protocols, which led to a slightly lower electric field strength (Figure 15C). However, this was a necessary step in order to match the amplitude of the envelope frequency in the amplitude modulation protocol to the amplitude of the theta frequency in the remaining protocols.

In order to estimate the magnitude of the induced electric field in the brain, we ran simulations using the free software package Simulations for Non-invasive Brain Stimulation (SimNIBS; version 3.0.2; Thielscher et al. (2015)). To this aim, we conducted electric field calculations on an anatomically realistic, six-compartment template head model available in SimNIBS. We used default conductivity values $[\mathrm{S} / \mathrm{m}]$ that were set to 0.465 for the scalp, 0.01 for skull, 1.654 for cerebrospinal fluid, 0.275 for gray matter and 0.126 for the white matter. The simulation accounted for volume-normalized anisotropy in the brain. We observed peak electric field magnitudes up to $0.3 \mathrm{mV} / \mathrm{mm}$ in the medial prefrontal cortex (Figure 15C). 


\subsubsection{Procedure}

At the start of each session, the participants filled out a short questionnaire. We asked our participants to report the quality of sleep during the previous night. Further, we assessed the level of arousal, the presence and intensity of headache, and the intake of medication, coffee or alcohol consumption in the 24 hours before the session. The purpose of this was to avoid the possibility that irregular sleep patterns in the previous night, headache or mental fatigue due to alcohol would corrupt the possible behavioral findings of tACS. A new session was scheduled if the participant had consumed more than two alcoholic beverages in the previous day, however arranging a new session was not necessary.

All participants received detailed written instructions about the task. Before the training session, we asked them to perform a practice session to familiarize themselves with the task and to ensure that they were able to operate the response box (RB-740, Cedrus Corporation, USA) comfortably. We used an independent set of cards in the practice session. Before the start of the learning task, the participants filled out a questionnaire to ensure that they understood the tasks correctly. The questionnaire assessed whether the participants understood i) the meaning of the three feedback types (win, no win/no loss, loss) and ii) the probabilistic nature of the feedback. In the following stimulation sessions, the short questionnaire was followed by the electrode preparation, the application of the topical anesthetic cream, and the impedance measurements. This preparation phase took approx. 35 - 40 minutes during which the participants watched documentary movies to maintain their vigilance.

Following the preparatory phase, the participants performed two short practice tasks. The practice tasks contained 16 trials for the learning and 12 trials for the transfer phase.

Following the practice task and directly before the start of the learning task, the data collector opened the sealed envelope containing the information about that day's stimulation condition. After opening the envelope, the data collector selected the protocol on the stimulator and informed the participants about the start of the stimulation. Following this moment, the data collector initiated no further communication. The learning phase began directly after the fade-in period. After the end of learning phase and following a 5-minute break, the participants completed the transfer phase of the task, during which no stimulation was applied.

At the end of each session, we assessed the level of self-reported arousal, the presence and in- 
tensity of headache and secondary perceptual adverse effects associated with the application of tACS. We focused on cutaneous (i.e., itching, tingling and burning) and visual flickering sensations (i.e., phosphenes). First, the participants were asked to indicate the presence of secondary adverse effects (yes or no question). In case of a positive answer, we assessed the subjective level of discomfort using a 10-point Likert scale. On the Likert scale, "1" indicated the lowest noticeable discomfort and "10" indicated an amount of discomfort the participants would not be able to endure during the experiment. The participants were informed that they could discontinue the study at any time without having to give any reason for terminating the study.

At the end of each session, we asked our participants to recall the card types and provide an internal ranking of the cards. We focused on whether the participants were able to correctly recall the cards' valence-action contingency.

\subsubsection{Statistical analysis}

All statistical analyses were performed using the R statistical programming environment (version 3.5.1) and the RStudio integrated development environment (version 1.1.456; R Core Team (2018); Team and Others (2015)). For the data analysis, we used a Precision 7920 Rack computer, Debian GNU/Linux 9.9 operating system, $2 \times$ Intel Gold 6152, 2.1GHz, 22 cores and 512 GB RAM.

We applied Bayesian methods, and we report our results in terms of the mean of the posterior distribution and their associated 95\% highest-density intervals (HDI). The interpretation of the Bayesian 95\% HDI is that it gives the range in which the estimated parameter is located with a probability of 0.95 . We consider effects to be statistically reliable, if the $95 \%$ HDI excludes zero.

In order to model accuracy on the single-trial level, a dichotomous dependent variable, we used hierarchical Bayesian logistic regression. For these regression analyses, we used the $\mathrm{R}$ package brms (Bayesian Regression Models using Stan; Bürkner (2017)) with default, uniform priors for all regression coefficients. This package uses Hamiltonian Monte-Carlo (HMC) techniques implemented in Stan to fit the models (Carpenter et al., 2017). We used four chains, where each chain had a warm-up period of 1,000 samples and 1,000 post warm-up samples resulting in a total of 4,000 posterior samples. We used the Gelman-Rubin diagnostic (Gelman et al., 1992) to ensure that all reported results had an $\hat{R} \leq 1.05$. For model comparison, we used the Leave-One- 
4 THETA-GAMMA CROSS-FREQUENCY TRANSCRANIAL ALTERNATING CURRENT STIMULATION OVER THE TROUGH IMPAIRS COGNITIVE CONTROL

Out Information Criterion (LOOIC), where lower scores of the LOOIC suggest a better model fit (Vehtari et al., 2017). Specifically, a model was considered better if the LOOIC score were lower, and if the $\triangle \mathrm{LOOIC}$ score were at least double the corresponding LOOIC standard error.

\subsubsection{Computational modelling}

The orthogonal Go-NoGo task used in our study usually allows one to fit computational reinforcement learning (RL) models to the data collected during the experiment (e.g., Cavanagh and Frank (2014); Csifcsák et al. (2020)). These models assume that each time a certain stimulus is encountered, an internal value representation of the stimulus-action pair (known as Q-value) is updated according to the reward received after taking an (in-) action. Furthermore, the decision on which action to take is based on this internal value-representation, and thus, as the Q-value gets close to the actual value with repeated encounters of a stimulus, performance becomes more accurate. The orthogonalized nature of the Go-NoGo task typically also allows the estimation of Pavlovian influences on this RL process by biasing Go-responses for rewarding stimuli and NoGo-responses for punished stimuli. We used Bayesian hierarchical modeling to fit a series of these models to our data using a strategy identical to that presented in Csifcsák and colleagues (2020), and we refer the reader to this paper, our supplemental materials and the data-repository for this paper at https://github.com/ihrke/2020_cfc_tacs for technical details of the RL model.

The described computational models were implemented using the R-package rstan (Stan development, 2016). We used eight parallel chains with a total of 8,000 post-warm up samples from the posterior distribution. The convergence diagnostics were identical with the other models as described above.

\subsection{Results}

\subsubsection{Computational modeling}

We fitted models of increasing complexity to the data from our experiment. First, we fitted a model without any session-specific terms (null-model) as a baseline. Next, we modeled separate learning-rates $\alpha$, temperature parameters $\beta$, Pavlovian bias parameters $\pi$ and go-biases $b$ for each of the tACS sessions (tACS-model). 
Furthermore, we included a model that let each of the four core-parameters depend on the session order (order-model) and, finally, a model where separate parameters were fit for each tACS session and each parameter depended on session-order (full model). Diagnostics of the HMC chains indicated that all models converged successfully.

We calculated the LOOIC for each of these models (see Table 1). Even though the model that only modeled the RL parameters as a function of session order received the lowest LOOIC, the differences between all four models were small compared to their standard errors (see Table 1) and model selection was therefore inconclusive. We conducted posterior predictive checks and simulated 1,000 random datasets from the posterior distribution of the parameters. Unfortunately, while some general characteristics of our participants' performance was captured by the model, it failed to properly account for the complex changes across sessions, trials and card types (for more detail, see Supplemental Figure 19). Given that the computational models were unable to capture our participants' behavior, we chose not to interpret or report changes in model parameters across sessions but to focus on the more descriptive logistic regression models reported below. The reason for our failure to model our participants' performance with these established models is puzzling and deserves further investigation.

Table 12: Results of the model selection procedure for the computational models. All differences in LOOIC are small compared to their standard errors and model selection is therefore inconclusive.

\begin{tabular}{llll}
\hline Model & LOOIC & $\Delta$ LOOIC & SE $(\Delta$ LOOIC $)$ \\
\hline Order & 10598.3 & - & \\
Full & 10607.8 & 9.6 & 30.2 \\
tACS & 10608.5 & 10.2 & 39.4 \\
Null & 10615.3 & 17.0 & 33.2 \\
\hline
\end{tabular}

SE: Standard error.

\subsubsection{Accuracy and learning}

To assess learning performance across sessions, we fitted a series of hierarchical Bayesian logistic regression models, treating accuracy as the dependent variable. All of the models received a random intercept for each participant and for sessions nested within participants. Furthermore, we included various combinations of the following predictor variables: Card-type (four levels: Go-to-Win, NoGo-to-Avoid, Go-to-Avoid and NoGo-to-Avoid), tACS-session (five levels: 
Training, Control, AM, Peak and Trough), Trial (Z-transformed trial number during each experimental session), Session order (continuous predictor coding for the order in which the tACS sessions were conducted) as well as their interactions. All of these 20 models (for a full list, see Supplemental Figure 20) were compared according to their out-of-sample predictive performance using the LOOIC (Vehtari et al., 2017). Based on this criterion, we calculated model weights using two different techniques: Based on Akaike weights (Wagenmakers and Farrell, 2004) using the LOOIC instead of the AIC and using Bayesian model averaging (Yao et al., 2018). Both of these techniques resulted in posterior probabilities quantifying how likely it is that each of the models was the best one.

After calculating these model selection criteria, we found converging evidence that the model that encompassed all predictors, including all two-way and three-way interactions between Card, Session and Trial, as well as a main effect of session order outperformed the other models (Akaike weight $p=0.63$, next best model $p=0.34$; Bayesian model averaging weight $p=0.47$, next best model $p=0.23$ ).

We therefore based our conclusions on that winning model and investigated it in detail. First, we checked that the model captured the trends in the data well. In Figure 16, we plotted the raw data and overlaid predictions from the winning logistic regression model (posterior predictive check). For more details, see Supplemental Figures 21 and 22. The model captured the trends in the data well and the uncertainty (95\% HDIs) around the model-predictions was sufficiently broad relative to the fluctuations in the data. The Bayesian value $R^{2}$ for this model was $R^{2}=0.23$ HDI $[0.22,0.24]$.
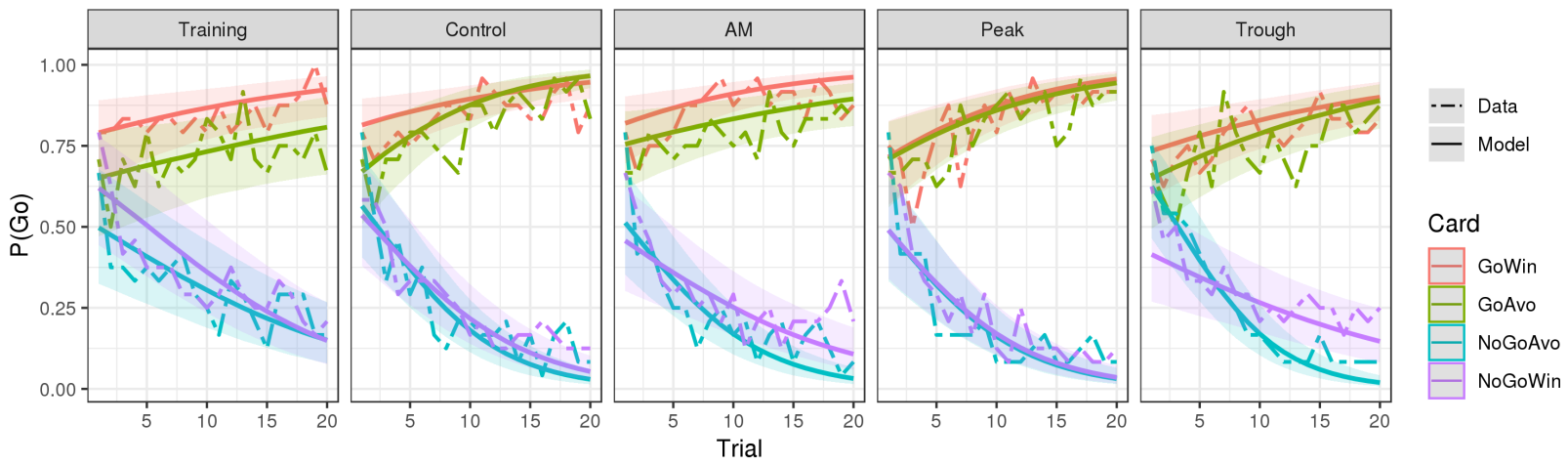

Figure 16: Posterior predictive checks for the final logistic regression model. The model predictions (solid lines) captured the main trends in the data (dashed lines) well. Colored ribbons are 95\% HDIs. Abbreviations: AM amplitude-modulated. 
We focused on two separate aspects of the data: First, we investigated how the general accuracy level varied across cards and sessions. In the presence of the three-way interaction of card $\times$ tACS-session $\times$ trial, we quantified and compared the accuracy level in the middle of each session. Second, we were interested in the learning rate with which accurate responding increased. In our model, this was manifested in the tACS-session $\times$ trial, card $\times$ trial and card $\times$ tACSsession $\times$ trial interactions that allowed us to investigate the rate with which the correct way to respond to each of the cards was learned across the sessions.

\subsubsection{Average accuracy}

The accuracy levels as estimated by the model in the middle of each session are displayed in Figure 17. There was a significant amount of variation both between the cards and sessions. As expected, responses to the Go-to-Win card were generally most accurate $\left(b_{\text {GoAvo }}=-.88\right.$ $[-1.24,-0.54], b_{\text {NoGoAvo }}=-1.02[-1.39,-0.68], b_{\text {NoGoWin }}=-1.25[-1.63,-0.90]$; these coefficients reflect the situation in the training session due to the presence of higher-order interactions (for full analyses, see Supplemental Figure 23) while the NoGo-to-Win condition was most difficult with the other two cards being situated between. Furthermore, we found a learning effect between the training session (which was always the first session each participant was exposed to) and the other sessions (which were randomized): Performance was better in all tACS sessions and for all cards (see Supplemental Figure 23), the only exception being the Go-to-Win card in the Trough condition $(P($ Trough $>$ Training $\cup$ GoWin $)=0.23)$. This learning-effect was not surprising given that this task is known to exhibit between-session learning effects (Csifcsák et al., 2020). However, after the initial effect of learning from the training session to the second one, there was no clear further effect of session order, $b_{\text {order }}=-0.08[-0.26,0.10]$. 


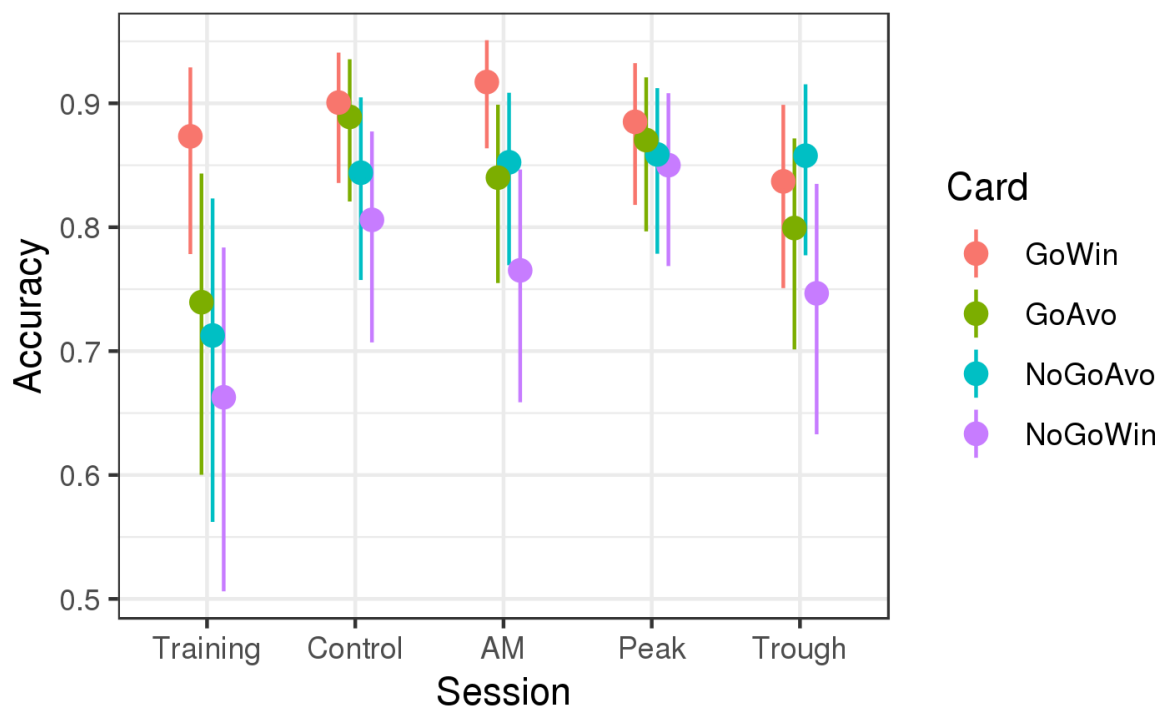

Figure 17: Estimated accuracy levels in the middle of the experimental session for each session and card. The colors represent the four card types, with the experimental sessions shown the horizontal axis. Note that the participants received no tACS in the training session. Abbreviations: AM - amplitude-modulated tACS; control - control tACS.

We were interested in how general accuracy changed between the different tACS conditions. A summary of the results is presented in Figure 18, upper row (for all conditions, see Supplemental Figure 23). Here, each entry in the matrix documents the posterior probability that accuracy was increased from one session (A) to the next (B). High values close to 1 (red) indicate that session A was highly likely to show increased accuracy relative to session B while low values close to zero indicate the opposite. Intermediate values (grey) mean that the results are inconclusive for that particular comparison.

We start by comparing the three active tACS conditions "AM", "Peak" and "Trough" with the control condition. The $p$-values given here represent the posterior probability that the active session showed higher accuracy compared with the control condition (i.e., the probability that the difference $b$ is positive) and are not to be confused with frequentist p-values. There was no clear difference between the AM and the control condition for congruent cards (GoWin: $b=0.21[-0.47,0.87], p=0.73$, NoGoAvo: $b=0.06[-0.54,0.75], p=0.58)$ with possibly a small performance decrease for conflict cards (GoAvo: $b=-0.43[-1.09,0.19], p=0.10$, $b=-0.25[-0.85,0.38], p=0.22)$ though the HDIs for these effects did not exclude zero. The Peak condition did not result in a change in general accuracy compared with control for congru- 
ent (GoWin: $b=-015$ [-0.86,0.47], $p=0.32$, NoGoAvo: $b=0.11[-0.52,0.76], p=0.63$ ) or conflict cards (GoAvo: $b=-0.19[-0.84,0.47], p=0.28$, NoGoWin: $b=0.31[-0.32,0.95]$, $p=0.84)$. Finally, the Trough condition showed reduced accuracy particularly for the easiest Go-to-Win condition $b=-0.56[-1.18,0.11], p=0.05$, (but not for NoGo-to-Avoid, $b=0.11$ $[-0.55,0.74], p=0.62$ ) and reduced accuracy for both conflict conditions (GoAvo: $b=-0.70$ $[-1.33,-0.04], p=0.02$, NoGo-to-Win: $b=-0.34[-0.95,0.29], p=0.14)$. Direct comparisons between the active stimulation conditions are also shown in Figure 18 (upper row).
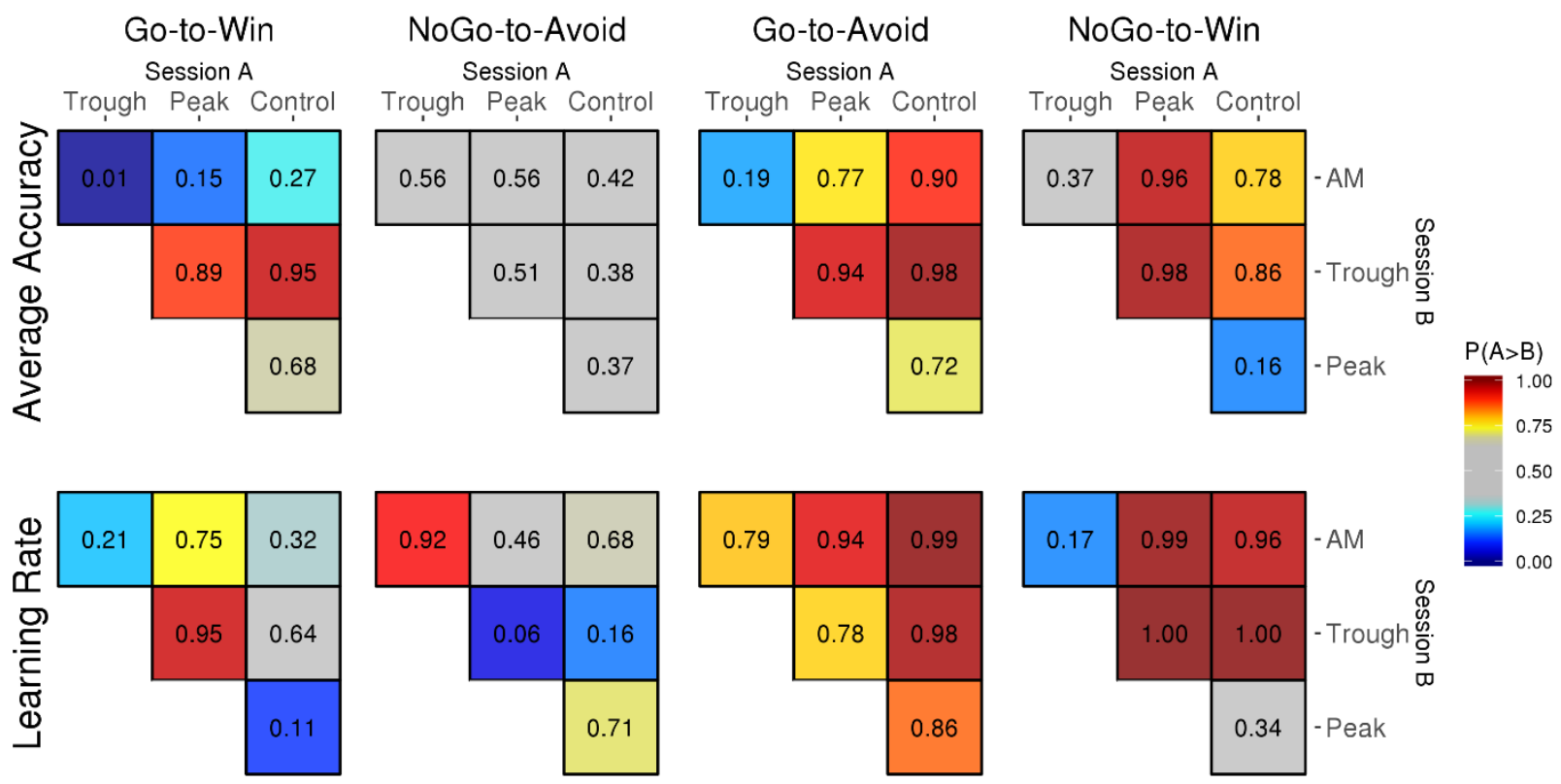

Figure 18: Comparison of average accuracy (top row) and learning rate (bottom row) between tACS-sessions for each of the four experimental conditions. Colors and numbers in the matrices indicate the probability that the session indicated by the column showed a stronger effect compared with the session indicated by the row of each matrix. Abbreviations: AM - amplitude-modulated.

\subsubsection{Learning rate analysis}

Next, we supplemented the analysis of the general accuracy with a parallel analysis regarding the learning rate, i.e., card and session interactions with the Trial term in the model. In Figure 18 the lower row shows a summary of this analysis. AM and control condition did not differ clearly for congruent cards (GoWin: $b=0.10[-0.30,0.52], p=0.68$, NoGoAvo: $b=-0.09$ $[-0.47,0.31], p=0.32$ ), but learning was decreased for conflict cards (GoAvo: $b=-0.50$ 
$[-0.88,-0.13], p=0.01$, NoGoWin: $b=-0.33[-0.69,0.02], p=0.04)$. For the Peak condition, the results are similar but less clear, with a possible small improvement for Go-to-Win cards $(b=0.24[-0.14,0.58], p=0.89)$ but not NoGo-to-Avoid $(b=-0.11[-0.51,0.28], p=0.29)$ and possibly a weak decrease for Go-to-Avoid cards $(b=-0.21[-0.61,0.18], p=0.14)$ but not NoGo-to-Win $(b=0.08[-0.30,0.44], p=0.84)$. For the Trough condition, we see no clear differences for congruent cards (GoWin: $b=-0.07[-0.41,0.31], p=0.36$, NoGoAvo: $b=0.21$ $[-0.22,0.59], p=0.84)$ but clear learning decreases for the conflicting cards (GoAvo: $b=0.36$ $[-0.01,0.74], p=0.02$, NoGoWin: $b=0.49[0.11,0.80], p=0.00)$.

\subsubsection{Perceptual adverse effects}

Most participants reported no cutaneous sensations during tACS, possibly due to the application of the topical anesthetic cream. However, we also inspected the amount of perceptual adverse effects, such as itching, tingling, and burning sensations, and phosphenes that were reported following each tACS session. A careful inspection of the subjectively reported perceptual adverse effects did not reveal any substantial differences between the stimulation sessions (for detail, see Supplemental Figure 25).

\subsection{Discussion}

In this study, we investigated the behavioral effects of three main types of theta-gamma CFCtACS protocols. We found that CFC-tACS over the peak had no consistent behavioral effects on the accuracy or learning rate compared with the control protocol. On the other hand, CFC over the trough protocols decreased the accuracy and the learning rate in the conflicting trials, i.e., in the NoGo-to-Win and Go-to-Avoid decisions. Furthermore, the amplitude-modulated tACS protocol reduced the learning rate but not the accuracy in the conflicting trials.

As we had hypothesized we found that the theta-gamma CFC-tACS over the through impaired behavioral performance, in particular in the conflicting trials. At the same time, the participants improved in one of the congruent trials, i.e., NoGo-to-Avoid. This suggests that CFC-tACS over the trough reduced the performance for the challenging trials and improved the learning rate in the easy trials. The direction of our results is in line with those found by de Lara et al. (2018), who used a long-term memory recall task and similarly found a decreased performance in CFC- 
tACS over the trough. We speculate that CFC-tACS over the trough likely interfered with the phase-dependent theta-gamma coupling between the cingulate (e.g., ACC) and the prefrontal cortices (e.g., DLPFC; Smith et al. (2015)). Thus, the modulation of the information flow from the cingulate to prefrontal cortex via theta-gamma coupling could have impaired the modelbased control in the trough protocol.

Unexpectedly, the amplitude-modulated CFC-tACS protocol slowed the learning rate for the conflicting cards, which is reminiscent of the behavioral effects of the CFC-tACS over the trough protocol. However, its diminishing behavioral effect was less pronounced when compared with the CFC-tACS over the trough protocol. In the amplitude-modulated tACS protocols, the slow, i.e., the theta frequency, might have played an important role in producing the cognitive effects of tACS (Minami and Amano, 2017). As increased power of theta-range oscillations leads to better performance during cognitive conflict (Cavanagh et al., 2013), we would expect behavioral improvement under this protocol. Previous studies with single-frequency theta tACS showed beneficial behavioral effects in cognitive control tasks, including reduced reaction time or facilitated behavioral accuracy (Hsu et al., 2017; Lehr et al., 2019).

Contrary to our expectations, we found no clear and consistent behavioral effects for the CFCtACS over the peak protocol. In a previous study, Alekseichuk et al. (2016) observed behavioral improvement in the sensitivity index of a spatial working memory task during CFC-tACS over the peak. Since the CFC-tACS peak protocol mimics the phase-specificity of theta-gamma CFC when signaling the need for cognitive control (Smith et al., 2015), we expected that it would increase the efficacy of the cingulate cortex to signal the need for cognitive control and thereby increase the degree of model based control implemented by the prefrontal cortex. The lack of the behavioral effects could also have been due to the thorough instructional procedure we used in the present study. The exhaustive instructional procedure might have produced a ceiling effect, which could diminish the ability of the stimulation to further improve the performance of our volunteers. We expect that the CFC-tACS over the peak protocol may improve the behavioral performance in groups of participants who do not reach the ceiling effect, e.g., in elderly participants or in individuals with mild cognitive impairment.

One of the limitations of the present study is that the computational modelling results were inconclusive given that the model was unable to capture our participants' behavior. Therefore, we can neither confirm nor falsify our third hypothesis concerning the underlying cognitive pro- 
cesses (i.e., Pavlovian bias parameter). We speculate that the lack of fit of our computational models could be, at least partially, due to the instructional procedure we used in this study. Specifically, our participants received very thorough instructions about the task including reading the written instruction, listening to the verbal explanation of the experimenter, performing the short practice, filling out the questionnaire about the task, and performing the training session. By this procedure, we initially intended to minimize the probability that the participants would misunderstand the task and make their decisions in a random fashion. However, the exhaustive instructional procedure likely affected the strategy of the participants, who performed very well on the task. In fact, even though our task was more difficult than that used in previous studies (e.g., in Cavanagh et al. (2013)), the overall accuracy level in the tACS sessions was higher in our study indicating that the subjects were potentially able to exploit the task structure to improve their reward rate.

Evidence exists that the task instruction can indirectly influence how humans perform an instrumental learning task. This phenomenon is known in the literature as the behavioral rulegoverning effect (Doll et al., 2009). It is possible that after the instructional phase at least some participants were able to infer the correct structure of the task, even before the direct experience. This may have facilitated the learning process through the mechanism of confirmation bias (Doll et al., 2009); participants learned quickly to amplify those outcomes that were consistent with their internal model of the task and discarded the incompatible ones. Given the relatively difficult reward contingency probabilities ( 0.65 vs 0.35 ), we expected much more exploration in the initial phase of the task (e.g., Csifcsák et al. (2020)).

This argument is further supported by the results of the qualitative analysis we performed about the explicit knowledge of the card types (for further details, see Supplemental Note in 4.6). We found that all participants were able to correctly identify both the valence and the action value of the cards in the overwhelming majority of the cases (approx. 91\%). Occasionally, the participants made mistakes when identifying the correct action to the valence (approx. 8\%). Other error types were very rare. We interpret these findings as a further indirect support that the participants had explicit, rule-based knowledge about the structure of the task.

By using a less thorough instructional procedure, future studies may use computational modeling (e.g., in Csifcsák et al. (2020)) to explore the hidden parameters that may be influenced by the CFC-tACS protocol. Because these models assume that participants do gradually learn the 
expected value of the stimulus (Cavanagh et al., 2013; Csifcsák et al., 2020), we were not able to utilize them fruitfully in the present study.

Another possible limitation of the present study is the lack of a sham tACS protocol. Because real tACS can induce both cutaneous and visual perceptual adverse effects during the entire stimulation period, we preferred using a control tACS protocol, instead of a sham tACS protocol (Turi et al., 2013). The conventionally used fade-in, short-stimulation, fade-out sham protocols, may not be able to maintain effective blinding for the real intervention due to their shortness, as has been shown for transcranial direct current stimulation (Greinacher et al., 2019; Turi et al., 2019).

Taken together, CFC-tACS protocols can extend single-frequency tACS protocols by enabling the testing of CFC phenomena intrinsic to endogenous network oscillations (Alekseichuk et al., 2016; Bächinger et al., 2017; Minami and Amano, 2017). In this study, we showed that CFCtACS over the trough, and amplitude-modulated tACS decreased the behavioral performance and the use of cognitive control in healthy participants. These findings suggest that the phase of coupling between theta and gamma frequencies may play an important role in cognitive control.

\section{Disclosure}

The funding sources had no role in study design, collection and analysis of data, decision to publish, or drafting the manuscript.

\section{Conflicts of interest}

The authors report no conflict of interest.

\section{Acknowledgments}

The authors wish to thank Dr. med. Anja Manig, Dr. med. Sebastian Schade, Dr. med. Dirk Czesnik and Dr. med. Claire Halsband for the neurological examinations. We thank Prof. Thomas Crozier for his comments on the manuscript. 


\section{Funding sources}

This research was financially supported by DFG PA 419/15-1 awarded to WP. AL was supported by the IMPRS Neurosciences.

\subsection{Supplemental information}
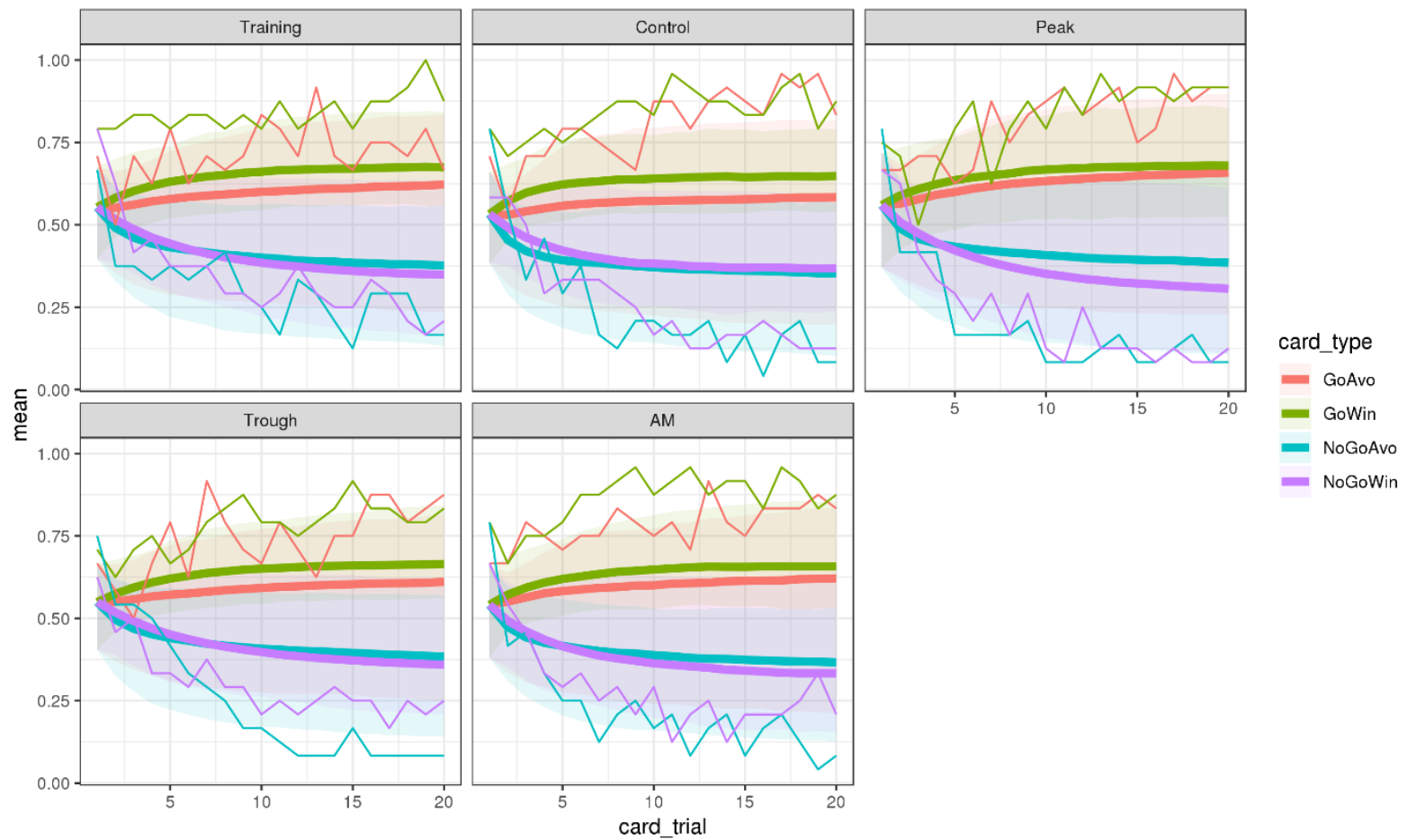

Figure 19: Computational modelling results. Although the model converged successfully, posterior predictive checks revealed a misfit between model prediction and observed data. Related to Table 12. 
4 THETA-GAMMA CROSS-FREQUENCY TRANSCRANIAL ALTERNATING CURRENT STIMULATION OVER THE TROUGH IMPAIRS COGNITIVE CONTROL

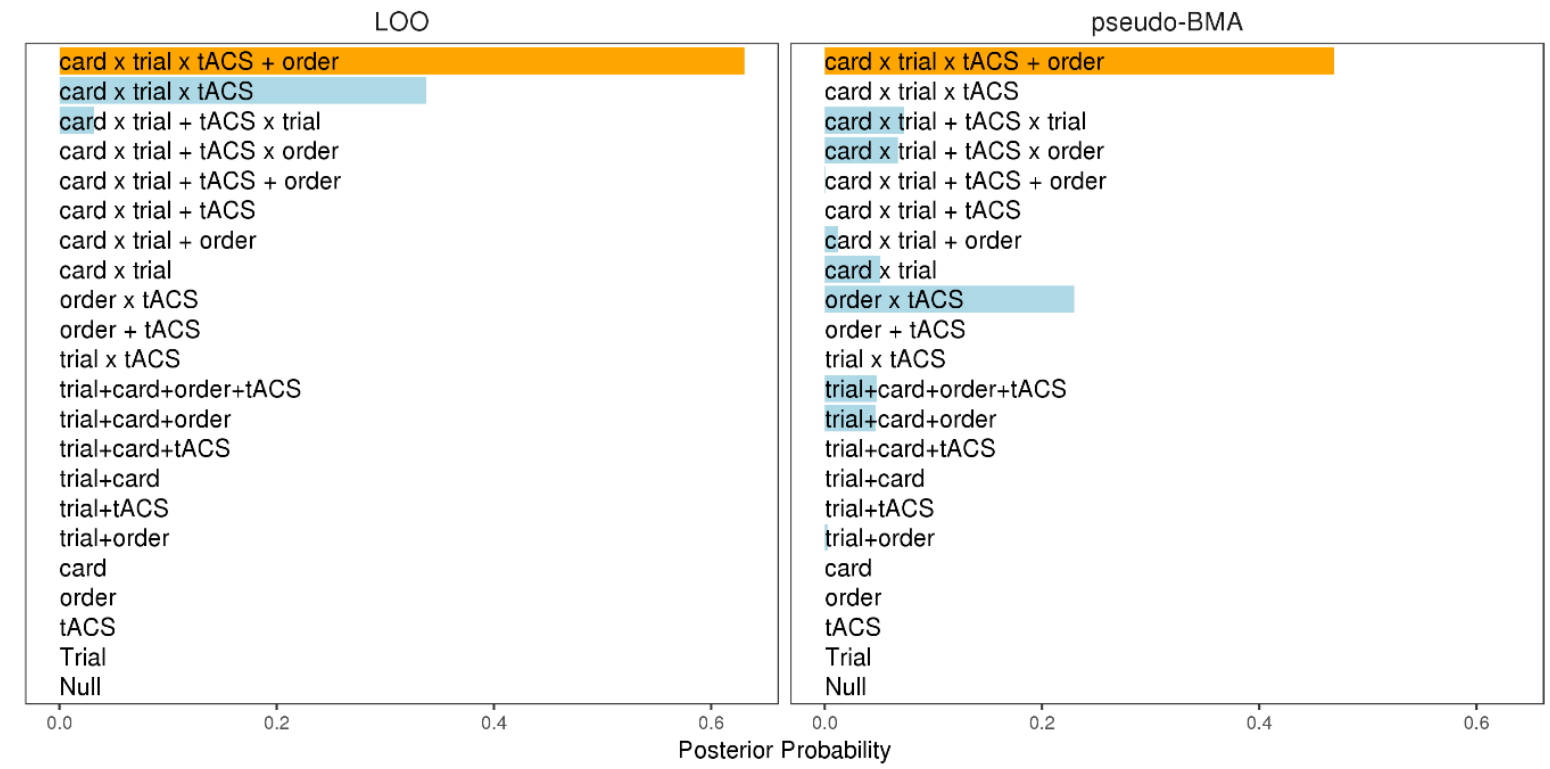

Figure 20: The model selection procedure for all computational models. The procedure favored the full model with the three-way interaction between card, trial and tACS, and the main effect of order. Both the leave-oneout information criterion and pseudo Baysian model-averaging estimated this model to have the highest posterior probability of being the best model. Related to Figure 16.
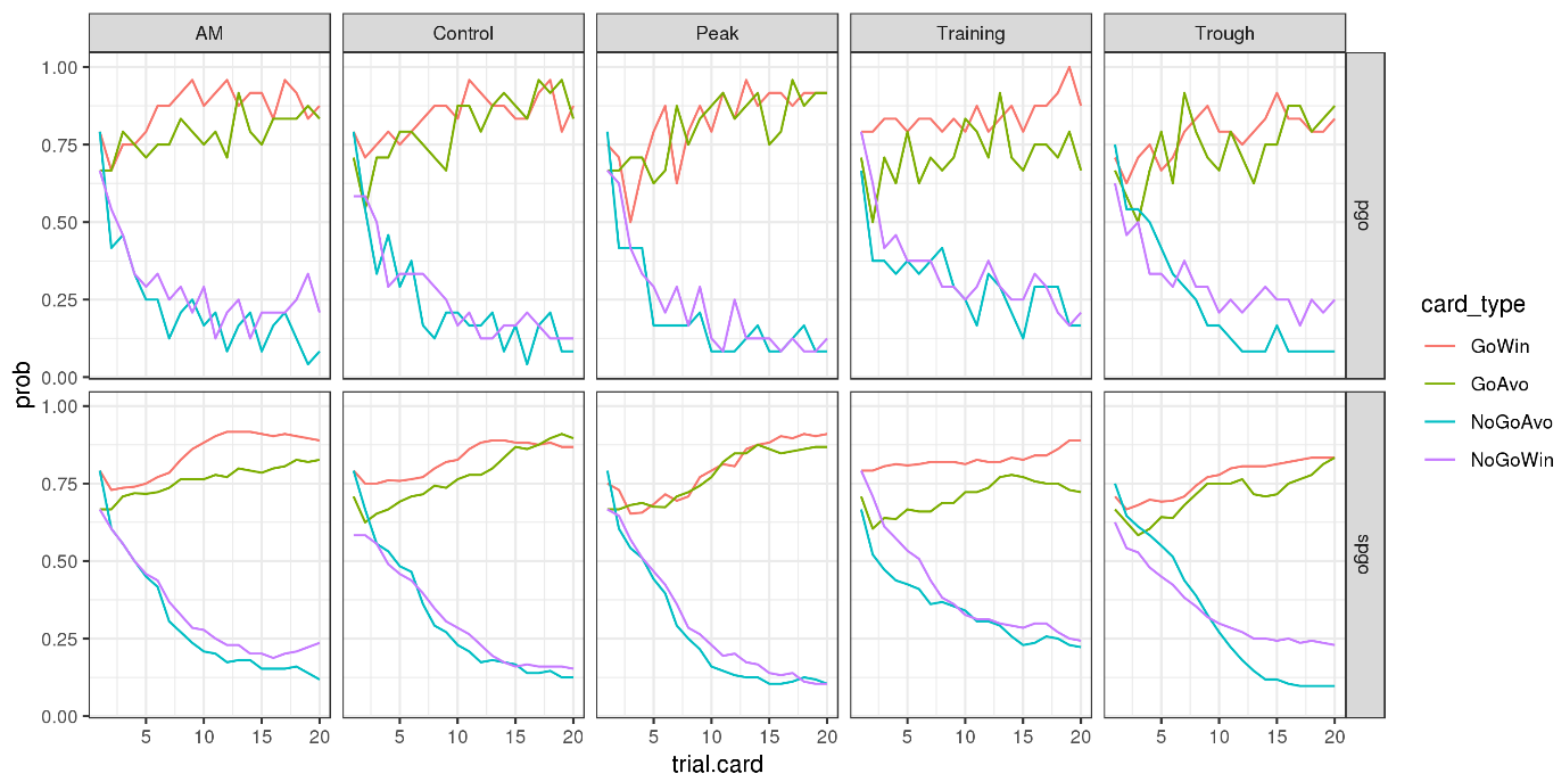

Figure 21: Probabilities of Go-responses. Average (across participants) probability to give a Go-response to each card per trial and session (top) and the same but smoothed with a five-trial running average window (bottom). Related to Figure 16. 
4 THETA-GAMMA CROSS-FREQUENCY TRANSCRANIAL ALTERNATING CURRENT STIMULATION OVER THE TROUGH IMPAIRS COGNITIVE CONTROL
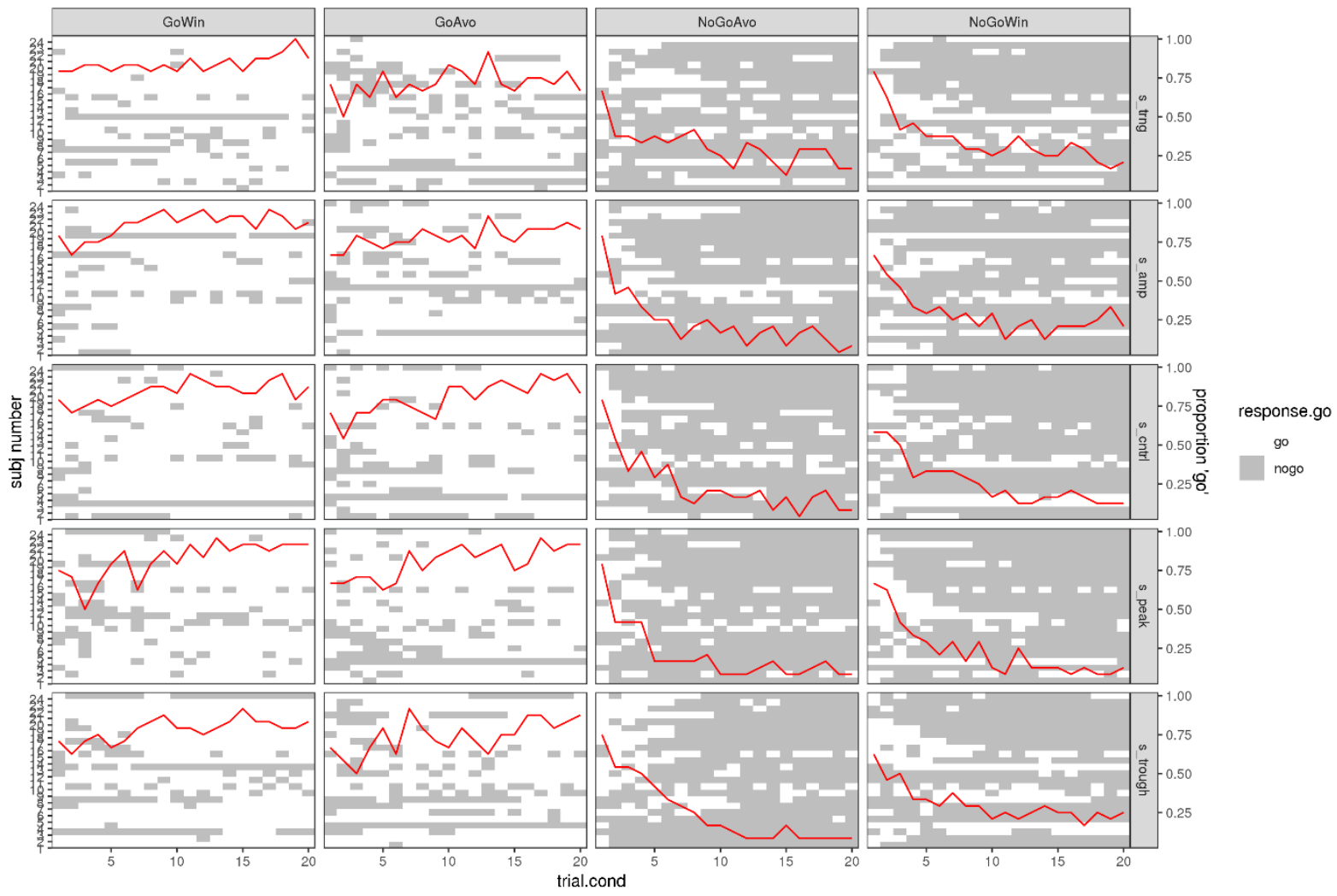

Figure 22: All individual responses. The Go (white) and NoGo (grey) responses over the 20 trials shown in the four card types (columns) and five experimental conditions (rows). Red line shows the mean performance calculated over the 24 participants. Related to Figure 16.
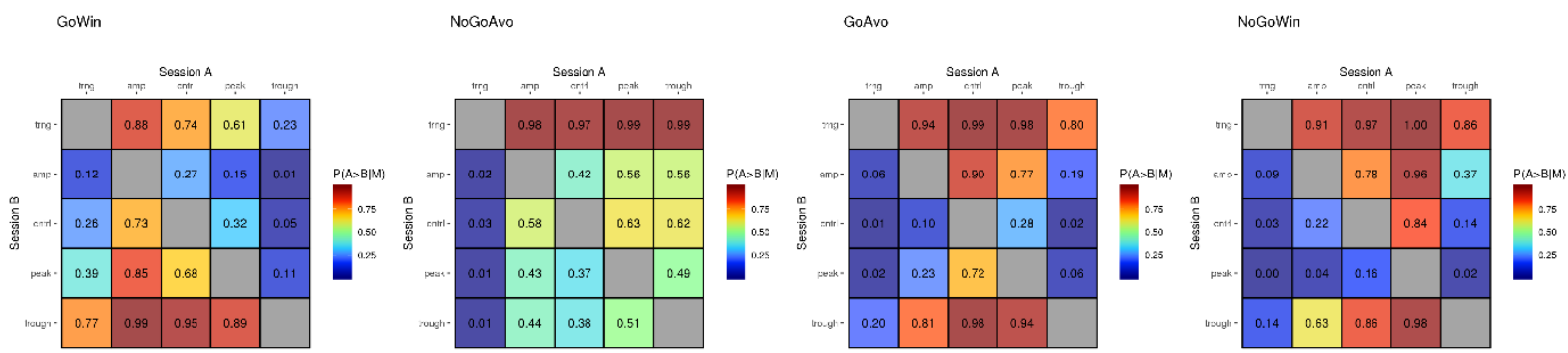

Figure 23: Comparison of average accuracy between all sessions for each of the five experimental conditions. Colors and numbers in the matrices indicate the probability that the session indicated by the column showed a stronger effect compared with the session indicated by the row of each matrix. Related to Figure 18. 

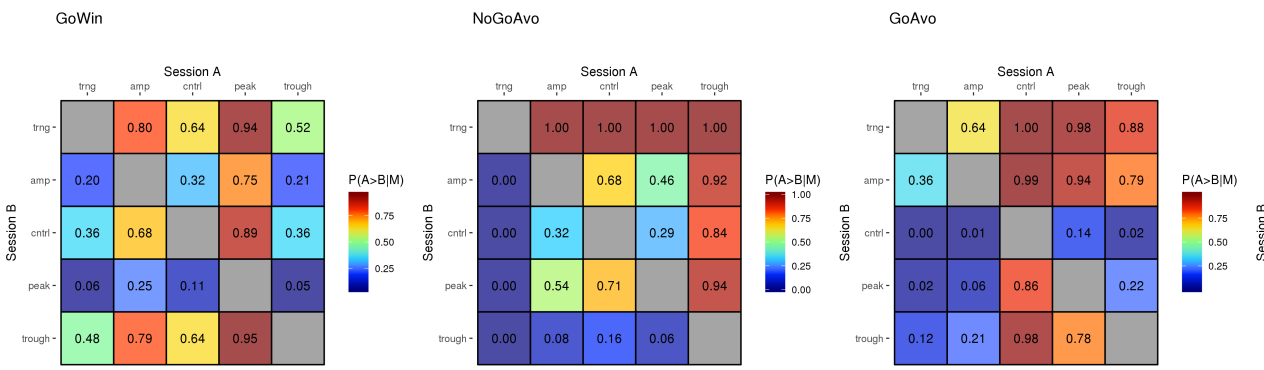

NoGoWin

Figure 24: Comparison of learning rate between all sessions for each of the five experimental conditions. Colors and numbers in the matrices indicate the probability that the session indicated by the column showed a stronger effect compared with the session indicated by the row of each matrix. Related to Figure 18.
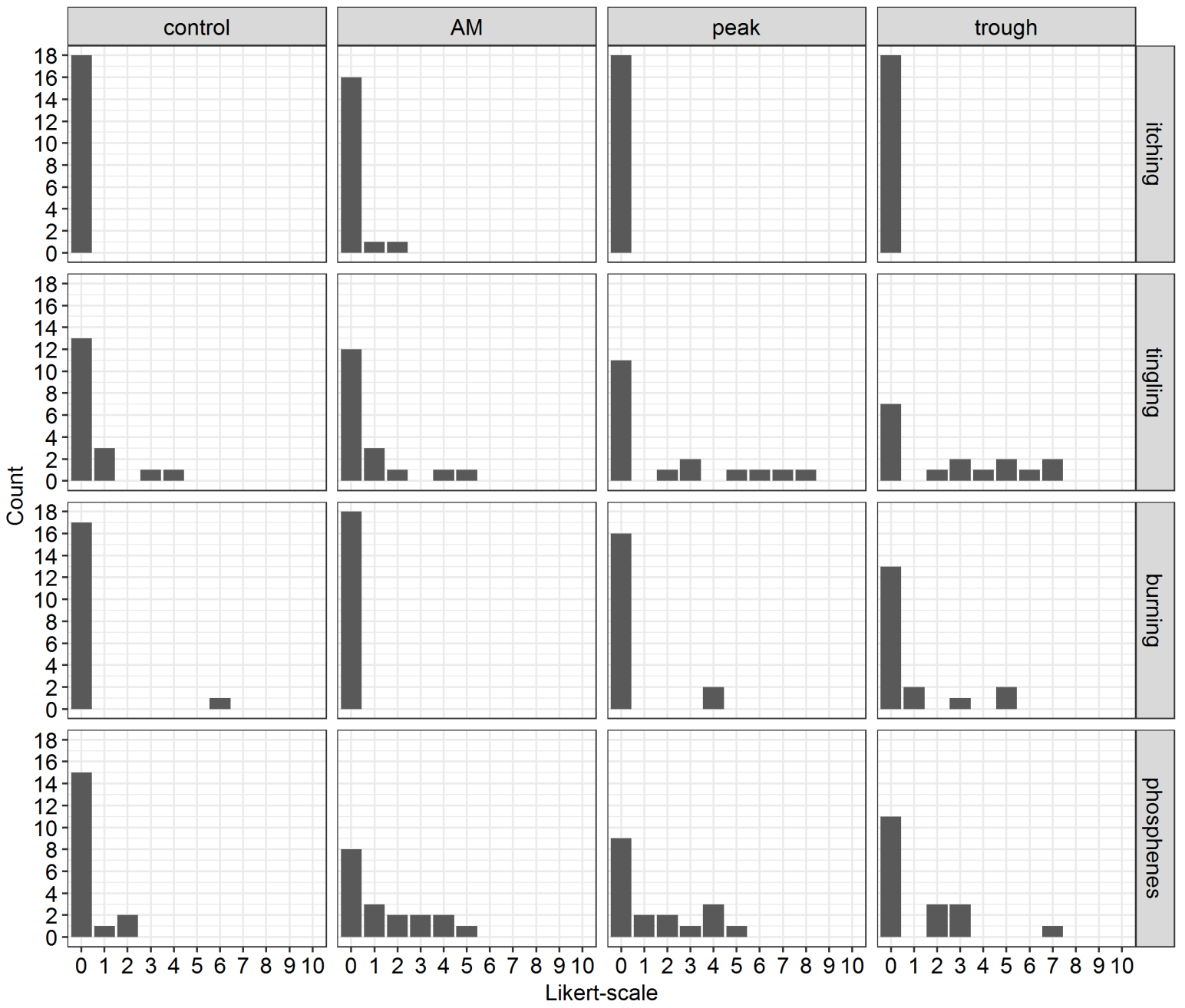

Figure 25: The participants reported a mild amount of perceptual adverse effects during the application of stimulation using a Likert-scale. Zero indicates no perceivable adverse effects, whereas "10" indicates an unbearable amount of discomfort. Note that only 18 participants provided the subjective reports, hence, the 'Count' axis is maximized at 18. Abbreviations: AM - amplitude modulated tACS. Related to Figure 18. 


\section{Supplemental note}

In all of the tACS sessions, every participant was able to correctly recall the letter associated with each card. We interpret these findings such that all participants attended to the task. In most cases (90.89\%), the participants were also able to correctly identify the exact card type, i.e., correctly recall both the valence and the action of the card. In $7.81 \%$ of the cases, they correctly identified the valence of the card but associated it with the incorrect action. An example for such an error is, when a participant identified a card as 'Go to win', although the card was 'NoGo to win'. Very rarely $(0.26 \% ; 1$ case $)$, one participant correctly identified the action associated with the card but the recalled valence was incorrect. This happened when a 'NoGo to win' card was identified as 'NoGo to lose' card. In $1.04 \%$ of the cases, participants were neither able to identify the valence nor the action associated with the card.

We also asked the participants to provide a subjective ranking of the cards. As expected, the 'Go to win' card received the best rank, followed by the 'NoGo to win' card. Participants ranked the 'Go to avoid losing' card at the third and the 'NoGo to avoid losing card' at the fourth rank. That is, the participants preferred winning cards over losing cards, and Go responses over response inhibition. This pattern of results is in agreement with the findings of previous studies (e.g., Cavanagh et al. (2013)).

The internal ranking also reflects the subjective difficulty related to the Pavlovian congruent and conflicting cards. The ranking was very clearly separable for the Go to win and NoGo to avoid losing cards. For these cards, the participants assigned more than $90 \%$ of the answers to the first or the last rank, respectively. For each of the Pavlovian conflicting cards, the participants distributed a substantial part of their answers between the second and third ranks. Although they still had a preference for the second and third ranks, this difference was less clear than for the Pavlovian congruent cards and may reflect the participants' uncertainty. 

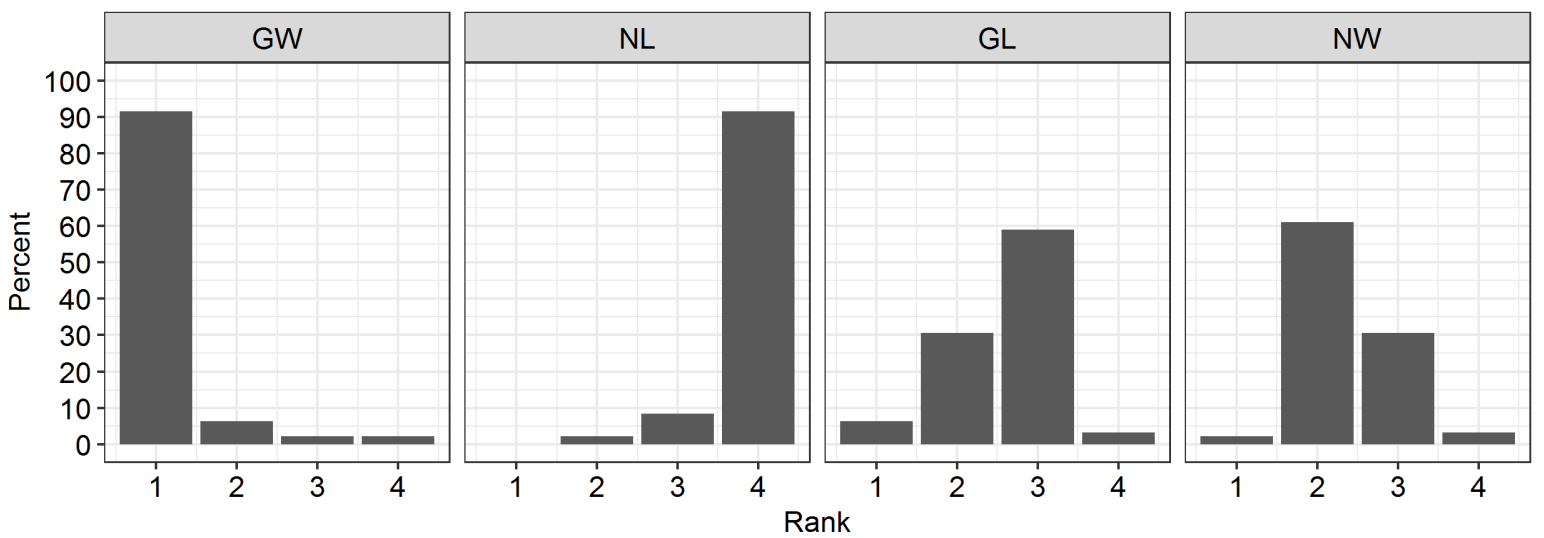

Figure 26: The subjective ranking of the cards. Go to win was most preferred, and NoGo to avoid losing least. NoGo to win was preferred slightly over Go to avoid losing. Related to Figure 18.

The participants ranked the 'Go to win' card most often in first place and the 'NoGo to avoid losing' card in fourth place. The 'NoGo to win' card was most often ranked second with the 'Go to avoid losing' card in third place. The difference in the ranking between these Pavlovian conflicting cards was smaller than for the Pavlovian congruent cards. 


\section{General Discussion}

Cognitive control is a psychological construct that is ubiquitous in complex human behavior but evades direct observation. A wide network of brain areas is correlated to the emergence of cognitive control. To separate the function of each brain area is experimentally challenging as the classical scientific method of inhibiting functions of parts of a system is not possible in cognitive neuroscience. tACS allows one to change activity in brain areas, even though complete cessation of activity cannot be achieved.

In my doctoral research, I studied the behavior of the CCN under different stimulation paradigms. In Chapter 2, mid-theta range tACS over the DLPFC reduced the behavioral Stroop effect. In Chapter 3, mid-theta range tACS targeting the dACC reduced the behavioral Stroop effect, but no clear effects persisted after stimulation. In Chapter 4, cross-frequency tACS, that nested gamma oscillations into theta oscillations and targeted the dACC, worsened cognitive control during a Go/NoGo instrumental learning task. In this chapter I discuss the commonalities of the results that were discussed in the three preceding chapters. Furthermore, I illustrate scientific limitations of tES.

\subsection{DLPFC and dACC causally underlie cognitive control}

The dACC is proposed arbitrator between increasing cognitive control to achieve optimal performance and minimizing (computational) effort. It assumes a key role in a hippocampal-striatalprefrontal network (Anderson et al., 2016). Resolution of Pavlovian conflicts depends on increased power in theta-range oscillations in ACC (Cavanagh et al., 2013), which leads to a preference towards model-based control because model-free control is performing (currently) inadequately. Theta power in ACC synchronizes the theta phase and modulates gamma power (CFC) in DLPFC in cognitively-straining tasks (Oehrn et al., 2014; Smith et al., 2019). Intracranial recordings during a Stroop-like cognitive interference task showed high gamma $(70 \mathrm{~Hz}-$ $125 \mathrm{~Hz}$ ) activity to be coupled cross-frequential to theta activity in both ACC and DLPFC (Smith et al., 2015). This gamma power was maximal between $0^{\circ}$ and $60^{\circ}$ phase of theta-range oscillation. The information encoded in gamma activity was shown to travel from ACC to DLPFC (Smith et al., 2015). This indicates the ACC as a monitor and the DLPFC as a controller of cognitive control (Shenhav et al., 2017; Smith et al., 2019). In Chapter 4, we show the effect 
of gamma power to be dependent on the phase of the theta-range oscillation. Thus, as dACC is known to be the generator of midline theta rhythm (Inanaga, 1998; Ishii et al., 1999), our results add evidence to the dACC being upstream of DLPFC.

A mechanistic link between cognitive control and arbitration between model-free and modelbased learning was proposed by Cavanagh et al. (2013). Increases in dACC theta power within instrumental learning could lead to increased activation of the subthalamic nucleus (STN) of the basal ganglia via the hyperdirect pathway (Cavanagh et al., 2011). This could act as a brake during decision conflict (Cavanagh et al., 2011, 2013). Disruption of the DLPFC led to decreased ability to employ model-based control for optimal performance (Smittenaar et al., 2013). The DLPFC projects (within the basal ganglia) mostly to the rostral region of the caudate nucleus (Haber et al., 2006; Haber, 2016), while the dACC projects extensively to both caudate nucleus and putamen (Haber et al., 2006). As model-based control is associated next to DLPFC and dACC with the caudate nucleus and model-free control with the putamen (Wunderlich et al., 2012). The projections fit the proposed role of the DLPFC as coordinator of cognitive control and the ACC as the monitor. This is corroborated by simultaneous functional coupling between dACC and both striatal areas during decision (Wunderlich et al., 2012). In summary, disrupting the information flow after conflict detection from dACC to DLPFC to the striatum would realistically favor model-free instead of situationally more appropriate model-based control.

Generally, the induction of changes in cognitive control was similar in the experiments described in Chapter 2 and Chapter 3, in which the effect of tACS during Stroop task was studied for the first time. Stimulation of both DLPFC and dACC suggested that both brain areas causally underlie the reduced Stroop effects that indicate increased control. Their central roles in the emergence of cognitive control is therefore more credible, however in both cases the Stroop effect was reduced roughly by a third compared to sham stimulation. However, to suggest that two-thirds of the Stroop effect might be induced by other, additional mechanisms and brain areas is not a parsimonious theory. Due to scientific limitations of tES, which are detailed below, I cannot exclude that stimulation protocols better targeted to either brain area or individual thetarange oscillation might have led to a complete annihilation of the Stroop effect. As mentioned above, Chapter 4 gives more evidence that the dACC is upstream of the DLPFC. The here demonstrated importance of the $\mathrm{dACC}$ for cognitive control underpins the notion of the $\mathrm{dACC}$ to be the central node of the CCN as suggested in the EVC theory by Shenhav et al. (2013). 
An additional objective of my thesis was to demonstrate whether deeper brain areas as the dACC can be successfully targeted using tES. While at the beginning of my doctoral project most tES studies targeted brain areas close to the surface of the brain (Polanía et al., 2018), more scientists achieved behaviorally effective stimulation of the dACC (Onoda et al., 2017; To et al., 2018). The studies in Chapter 3 and Chapter 4 therefore flank the development towards more diverse targets in cognitive neuroscience.

\subsection{Scientific limitations of tES}

Transcranial electrical stimulation is an easy-to-apply method that induces changes in brain physiology. Historically, careful experimental designs were necessary to measure the specific functions of brain areas. Transranial electrical stimulation promised to do away with laboriously correlating activity of brain areas to behavior. By externally applying current the activity can be controlled as a independent variable. This allows to infer not only correlation with but causality of behavior. Thus, in theory the function of any brain area could be measured in any carefully designed behavioral outcome variable.

In practice, tES research increased markedly since its inception in 2000, but it also comes with criticism (Polanía et al., 2018). As mentioned before, tES is easy-to-apply which has led to a growth in clinical and cognitive studies without its mechanism being fully understood. For clinical purposes, the demonstration of safety and efficacy of new treatments is sufficient.

For cognitive neuroscience inducing changes in specific brain areas results in more accurate models of this brain area's functional role. As activity induced tES spreads throughout functional networks (Reed and Kadosh, 2018; Liu et al., 2018; Karabanov et al., 2019), it remains doubtful if functions can be researched below network level.

Additionally, the effect sizes of tES studies are often low and the results cannot be replicated (Horvath et al., 2015; Emmerling et al., 2017). The effects of tES are variable within and between subjects (Chew et al., 2015; Huang et al., 2017). Biological and technical factors are proposed to drive this variability. As many factors such as gender and age, arousal and prior brain states, neuroanatomy and skull thickness all might contribute, the field is far from adequately explaining and minimizing the biological variability. Similarly, tES depends on multiple technical parameters (e.g. current strength, stimulation duration, montage, oscillation frequency), 
the possibilities are manifold but optimization is challenging. Many diverse cognitive functions from speech to mathematics were studied with tES, which spreads out the evidence thinly and discourages verification and deepening of results (Parkin et al., 2015).

Multiple strategies have been proposed to combat the criticism and further reproducibility and impact of tES research (Polanía et al., 2018; Bikson et al., 2018). The placement of electrodes, the blinding of the stimulation protocol and computational modeling of electric field strengths were deemed important during a workshop involving senior researchers (Bikson et al., 2018). On a more general note, higher sample sizes and appropriate control tasks will improve quality, while elucidating the mechanism of tES in the brain would increase its explanatory strength (Polanía et al., 2018).

Arguably, some scientific problems mentioned above are technical in nature and the key concept behind tES (controlling brain activity) remains valid. The widespread current flow in biological tissues will always result in imprecise or indiscriminate targeting of brain areas. However, alternative implementations using temporally interfering electric fields (Grossman et al., 2017) are currently developed. Realistically, as all variability in human brain physiology and anatomy can not be accounted for, the method has to become robust to variability to continue being scientifically valid.

A common theme throughout this thesis has been the possibility of using tES to not only correlate brain functions and behavior but to imply causation from brain function to behavior. Based on correlations observed in EEG or functional magnetic resonance imaging which are used frequently in cognitive neuroscience, targets for transcranial electrical stimulation are defined. By inducing electrophysiological changes in these targeted brain areas, behavior might change. Therefore, we deduce that the function of the brain area is somehow causal to the observed behavior. A weakness of tES as a scientific method is that the steps described above only serve in translating known correlations into proposed causality. Thus, only confirmatory evidence is generated. Popper's dictum that theories can only be definitely falsified, but never definitely confirmed implies that the search for falsifying but not for confirmatory evidence is scientific best practice. The Bayesian revolution in science dethrones this theory and states that also confirmatory evidence strengthens a theory based on fitting observations (Howson and Urbach, 1991).

However, the strength of a theory stems not so much from how well the observations are ex- 
plained by the theory (confirmatory evidence), but from how no observations are in direct contradiction of the theory (no disconfirming evidence). Showing that cognitive control changes if the $\mathrm{CCN}$ is stimulated and its activity changed is an observation explained by the theory. Showing that cognitive control does not change if the $\mathrm{CCN}$ is stimulated and its activity is changed would be disconfirming evidence and deal a heavy blow to the theory that the CCN underlies cognitive control. Why is tES not used to show that a behavioral output remains unchanged even after the acitivtiy of the brain areas proposed to cause the behavior was changed? The method is too unreliable and too susceptible to biological variation as mentioned above. Stimulating a brain area and observing no effect in behavior would therefore be no evidence for the brain area not underlying the behavior. One would assume that the tES did not induce any change in brain activity. With a technique guaranteed to induce neurophysiological changes in targeted brain areas, the absence of behavioral effects would indeed be evidence for no correlation between these brain areas' activity and the behavior. Until the reliability of tES improves, only confirmatory evidence can be gathered as was done in this thesis. Giving evidence for causation moves cognitive neuroscience forward, but is humbled by the true potential of techniques that reliably and from externally induces changes in brain activity of human volunteers and patients.

\subsection{General conclusions and perspectives}

The results presented in this thesis added confirmatory evidence to the proposition that DLPFC and dACC causally underlying cognitive control. Their joint activity was shown to depend on theta-range neuronal oscillations generally and theta-gamma cross-frequency coupled oscillations in particular. Behaviourally effective stimulation of the dACC proves that brain areas beyond the brain's surfaces are realistic targets for tES.

While this does not inoculate the current theories against eventual falsification with contradicting observation, newer theories have to account for this evidence. In future research the tight functional coupling between DLPFC and dACC across time should be further inspected. Ascribing them clearly defined functions throughout every time increment will be the basis for classifying the functional importance of further hubs of the CCN. 


\section{Bibliography}

Alekseichuk, I., Z. Turi, G. Amador De Lara, A. Antal, W. Paulus Correspondence, and W. Paulus

2016. Spatial Working Memory in Humans Depends on Theta and High Gamma Synchronization in the Prefrontal Cortex. Current Biology, 26:1-9.

Alexander, W. H. and J. W. Brown

2011. Medial prefrontal cortex as an action-outcome predictor. Nature Neuroscience, 14(10):1338-1344.

Ali, M. M., K. K. Sellers, and F. Fröhlich

2013. Transcranial Alternating Current Stimulation Modulates Large-Scale Cortical Network Activity by Network Resonance. Journal of Neuroscience, 33(27):11262-11275.

Ambrus, G. G., W. Paulus, and A. Antal

2010. Cutaneous perception thresholds of electrical stimulation methods: Comparison of tDCS and tRNS. Clinical Neurophysiology, 121(11):1908-1914.

Amemiya, S. and A. D. Redish

2018. Hippocampal theta-gamma coupling reflects state-dependent information processing in decision making. Cell reports, 22(12):3328-3338.

Amiez, C., J. Joseph, and E. Procyk

2006. Reward Encoding in the Monkey Anterior Cingulate Cortex. Cerebral Cortex, 16(7):1040-1055.

Anderson, M. C., J. G. Bunce, and H. Barbas

2016. Prefrontal-hippocampal pathways underlying inhibitory control over memory. Neurobiology of learning and memory, 134:145-161.

Antal, A., I. Alekseichuk, M. Bikson, J. Brockmöller, A. Brunoni, R. Chen, L. Cohen, G. Dowthwaite, J. Ellrich, A. Flöel, F. Fregni, M. George, R. Hamilton, J. Haueisen, C. Herrmann, F. Hummel, J. Lefaucheur, D. Liebetanz, C. Loo, C. McCaig, C. Miniussi, P. Miranda, V. Moliadze, M. Nitsche, R. Nowak, F. Padberg, A. Pascual-Leone, W. Poppendieck, A. Priori, S. Rossi, P. Rossini, J. Rothwell, M. Rueger, G. Ruffini, K. Schellhorn, H. Siebner, Y. Ugawa, 
A. Wexler, U. Ziemann, M. Hallett, and W. Paulus

2017. Low intensity transcranial electric stimulation: Safety, ethical, legal regulatory and application guidelines. Clinical Neurophysiology, 128(9):1774-1809.

Antal, A., K. Boros, C. Poreisz, L. Chaieb, D. Terney, and W. Paulus

2008. Comparatively weak after-effects of transcranial alternating current stimulation (tACS) on cortical excitability in humans. Brain stimulation, 1(2):97-105.

Antal, A. and C. S. Herrmann

2016. Transcranial Alternating Current and Random Noise Stimulation: Possible Mechanisms.

Antal, A. and W. Paulus

2013. Transcranial alternating current stimulation (tACS). Frontiers in Human Neuroscience, $7: 317$.

Baayen, H., D. Bates, R. Kliegl, and S. Vasishth

2015. RePsychLing: Data sets from psychology and linguistics experiments. $R$ package version $0.0,4$.

Bächinger, M., V. Zerbi, M. Moisa, R. Polania, Q. Liu, D. Mantini, C. Ruff, and N. Wenderoth 2017. Concurrent tACS-fMRI reveals causal influence of power synchronized neural activity on resting state fMRI connectivity. Journal of Neuroscience, 37(18):4766-4777.

Banich, M. T., M. P. Milham, R. A. Atchley, N. J. Cohen, A. Webb, T. Wszalek, A. F. Kramer, Z.-P. Liang, V. Barad, D. Gullett, C. Shah, and C. Brown

2000. Prefrontal regions play a predominant role in imposing an attentional set': evidence from fMRI. Cognitive Brain Research, 10(1-2):1-9.

Barch, D. M., T. S. Braver, L. E. Nystrom, S. D. Forman, D. C. Noll, and J. D. Cohen 1997. Dissociating working memory from task difficulty in human prefrontal cortex. Neuropsychologia, 35(10):1373-1380.

Bates, D., R. Kliegl, S. Vasishth, and H. Baayen

2015. Parsimonious mixed models. arXiv preprint arXiv:1506.04967. 
Bates, D., M. Mächler, B. Bolker, and S. Walker

2014. Fitting linear mixed-effects models using lme4. arXiv preprint arXiv:1406.5823.

Bench, C. J., C. D. Frith, P. M. Grasby, K. J. Friston, E. Paulesu, R. S. Frackowiak, and R. J. Dolan

1993. Investigations of the functional anatomy of attention using the Stroop test. Neuropsychologia, 31(9):907-22.

Benes, F. M.

2010. Amygdalocortical Circuitry in Schizophrenia: From Circuits to Molecules. Neuropsychopharmacology, 35(1):239-257.

Bikson, M., A. R. Brunoni, L. E. Charvet, V. P. Clark, L. G. Cohen, Z.-D. Deng, J. Dmochowski, D. J. Edwards, F. Frohlich, E. S. Kappenman, K. O. Lim, C. Loo, A. Mantovani, D. P. McMullen, L. C. Parra, M. Pearson, J. D. Richardson, J. M. Rumsey, P. Sehatpour, D. Sommers, G. Unal, E. M. Wassermann, A. J. Woods, and S. H. Lisanby

2018. Rigor and reproducibility in research with transcranial electrical stimulation: An NIMH-sponsored workshop. Brain Stimulation, 11(3):465-480.

Blair, K., A. A. Marsh, J. Morton, M. Vythilingam, M. Jones, K. Mondillo, D. C. Pine, W. C. Drevets, and J. R. Blair

2006. Choosing the Lesser of Two Evils, the Better of Two Goods: Specifying the Roles of Ventromedial Prefrontal Cortex and Dorsal Anterior Cingulate in Object Choice. Journal of Neuroscience, 26(44):11379-11386.

Blanchard, T. C. and B. Y. Hayden

2014. Neurons in dorsal anterior cingulate cortex signal postdecisional variables in a foraging task. The Journal of neuroscience : the official journal of the Society for Neuroscience, 34(2):646-55.

Bolker, B. M., M. E. Brooks, C. J. Clark, S. W. Geange, J. R. Poulsen, M. H. H. Stevens, and J.-S. S. White

2009. Generalized linear mixed models: a practical guide for ecology and evolution. Trends in ecology \& evolution, 24(3):127-35. 
Bonnefond, M., S. Kastner, and O. Jensen

2017. Communication between Brain Areas Based on Nested Oscillations. eneuro, 4(2):ENEURO.0153-16.2017.

Botvinick, M. M.

2007. Conflict monitoring and decision making: reconciling two perspectives on anterior cingulate function. Cognitive, affective \& behavioral neuroscience, 7(4):356-66.

Botvinick, M. M., T. S. Braver, D. M. Barch, C. S. Carter, and J. D. Cohen 2001. Conflict monitoring and cognitive control. Psychological review, 108(3):624-52.

Botvinick, M. M., J. D. Cohen, and C. S. Carter

2004. Conflict monitoring and anterior cingulate cortex: an update. Trends in Cognitive Sciences, 8(12):539-546.

Botvinick, M. M., S. Huffstetler, and J. T. McGuire

2009. Effort discounting in human nucleus accumbens. Cognitive, Affective, \& Behavioral Neuroscience, 9(1):16-27.

Breslow, N. E. and D. G. Clayton

1993. Approximate Inference in Generalized Linear Mixed Models. Journal of the American Statistical Association, 88(421):9.

Brown, J. W. and T. S. Braver

2005. Learned Predictions of Error Likelihood in the Anterior Cingulate Cortex. Science, 307(5712):1118-1121.

Bürkner, P.-C.

2017. Advanced Bayesian multilevel modeling with the $\mathrm{R}$ package brms. arXiv preprint arXiv:1705.11123.

Bush, G., P. Luu, and M. I. Posner

2000. Cognitive and emotional influences in anterior cingulate cortex. Trends in Cognitive Sciences, 4(6):215-222. 
Buzsáki, G., C. A. Anastassiou, and C. Koch

2012. The origin of extracellular fields and currents EEG, ECoG, LFP and spikes. Nature Reviews Neuroscience, 13(6):407-420.

Buzsáki, G., N. Logothetis, and W. Singer

2013. Scaling Brain Size, Keeping Timing: Evolutionary Preservation of Brain Rhythms. Neuron, 80(3):751-764.

Cain, S. W., E. J. Silva, A.-M. Chang, J. M. Ronda, and J. F. Duffy

2011. One night of sleep deprivation affects reaction time, but not interference or facilitation in a Stroop task. Brain and Cognition, 76(1):37-42.

Canolty, R. T. and R. T. Knight

2010. The functional role of cross-frequency coupling. Trends in cognitive sciences, 14(11):506-15.

Cappelletti, M., E. Gessaroli, R. Hithersay, M. Mitolo, D. Didino, R. Kanai, R. C. Kadosh, and V. Walsh

2013. Transfer of cognitive training across magnitude dimensions achieved with concurrent brain stimulation of the parietal lobe. Journal of Neuroscience, 33(37):14899-14907.

Carp, J. and R. J. Compton

2009. Alpha power is influenced by performance errors. Psychophysiology, 46(2):336-343.

Carpenter, B., A. Gelman, M. D. Hoffman, D. Lee, B. Goodrich, M. Betancourt, M. Brubaker, J. Guo, P. Li, and A. Riddell

2017. Stan: A probabilistic programming language. Journal of statistical software, 76(1).

Carter, C. S., T. S. Braver, D. M. Barch, M. M. Botvinick, D. Noll, and J. D. Cohen 1998. Anterior Cingulate Cortex, Error Detection, and the Online Monitoring of Performance. Science, 280(5364):747-749.

Carter, C. S. and V. van Veen

2007. Anterior cingulate cortex and conflict detection: an update of theory and data. Cognitive, affective \& behavioral neuroscience, 7(4):367-79. 
Cattell, J. M.

1886. The time it takes to see and name objects. Mind, os-XI(41):63-65.

Cavanagh, J. F., I. Eisenberg, M. Guitart-Masip, Q. Huys, and M. J. Frank

2013. Frontal Theta Overrides Pavlovian Learning Biases. Journal of Neuroscience, 33(19):8541-8548.

Cavanagh, J. F. and M. J. Frank

2014. Frontal theta as a mechanism for cognitive control. Trends in Cognitive Sciences, 18(8):414-421.

Cavanagh, J. F., T. V. Wiecki, M. X. Cohen, C. M. Figueroa, J. Samanta, S. J. Sherman, and M. J. Frank

2011. Subthalamic nucleus stimulation reverses mediofrontal influence over decision threshold. Nature neuroscience, 14(11):1462.

Chein, J. M. and W. Schneider 2005. Neuroimaging studies of practice-related change: fMRI and meta-analytic evidence of a domain-general control network for learning. Cognitive Brain Research, 25(3):607-623.

Chew, T., K.-A. Ho, and C. K. Loo

2015. Inter- and Intra-individual Variability in Response to Transcranial Direct Current Stimulation (tDCS) at Varying Current Intensities. Brain Stimulation, 8(6):1130-1137.

Chudasama, Y., T. E. Daniels, D. P. Gorrin, S. E. Rhodes, P. H. Rudebeck, and E. A. Murray 2013. The Role of the Anterior Cingulate Cortex in Choices based on Reward Value and Reward Contingency. Cerebral Cortex, 23(12):2884-2898.

Cohen, M. X.

2014a. A neural microcircuit for cognitive conflict detection and signaling. Trends in Neurosciences, 37(9):480-490.

Cohen, M. X.

2014b. Analyzing neural time series data: theory and practice. MIT press. 
Cohen, M. X. and T. H. Donner

2013. Midfrontal conflict-related theta-band power reflects neural oscillations that predict behavior. Journal of Neurophysiology, 110(12):2752-2763.

Cole, M. W. and W. Schneider

2007. The cognitive control network: Integrated cortical regions with dissociable functions. NeuroImage, 37(1):343-360.

Collins, M. L., C. A. Nelson, and MITCogNet.

2001. Handbook of developmental cognitive neuroscience. MIT Press.

Csifcsák, G., E. Melsæter, and M. Mittner

2020. Intermittent absence of control during reinforcement learning interferes with Pavlovian bias in action selection. Journal of Cognitive Neuroscience, 32(4):646-663.

Curtis, C. E. and M. D’Esposito

2003. Persistent activity in the prefrontal cortex during working memory. Trends in cognitive sciences, 7(9):415-423.

Dao-Castellana, M. H., Y. Samson, F. Legault, J. L. Martinot, H. J. Aubin, C. Crouzel, L. Feldman, D. Barrucand, G. Rancurel, A. Féline, and A. Syrota

1998. Frontal dysfunction in neurologically normal chronic alcoholic subjects: metabolic and neuropsychological findings. Psychological medicine, 28(5):1039-48.

Davidson, D. J., R. T. Zacks, and C. C. Williams

2003. Stroop interference, practice, and aging. Aging, Neuropsychology, and Cognition, 10(2):85-98.

de Lara, G. A., I. Alekseichuk, Z. Turi, A. Lehr, A. Antal, and W. Paulus

2018. Perturbation of theta-gamma coupling at the temporal lobe hinders verbal declarative memory. Brain stimulation, 11(3):509-517.

de Lara, G. A., P. N. Knechtges, W. Paulus, and A. Antal

2017. Anodal tDCS Over the Left DLPFC Did Not Affect the Encoding and Retrieval of Verbal Declarative Information. Frontiers in Neuroscience, 11:452. 
Diamond, A.

2013. Executive Functions. Annual Review of Psychology, 64(1):135-168.

Dixon, M. L. and K. Christoff

2012. The Decision to Engage Cognitive Control Is Driven by Expected Reward-Value: Neural and Behavioral Evidence. PLoS ONE, 7(12):e51637.

Doll, B. B., W. J. Jacobs, A. G. Sanfey, and M. J. Frank

2009. Instructional control of reinforcement learning: a behavioral and neurocomputational investigation. Brain research, 1299:74-94.

Dosenbach, N. U., D. A. Fair, A. L. Cohen, B. L. Schlaggar, and S. E. Petersen

2008. A dual-networks architecture of top-down control. Trends in Cognitive Sciences, 12(3):99-105.

Dosenbach, N. U., K. M. Visscher, E. D. Palmer, F. M. Miezin, K. K. Wenger, H. C. Kang, E. D. Burgund, A. L. Grimes, B. L. Schlaggar, and S. E. Petersen

2006. A Core System for the Implementation of Task Sets. Neuron, 50(5):799-812.

Duncan, J. and A. M. Owen

2000. Common regions of the human frontal lobe recruited by diverse cognitive demands. Trends in neurosciences, 23(10):475-83.

Edwards, D., M. Cortes, A. Datta, P. Minhas, E. M. Wassermann, and M. Bikson

2013. Physiological and modeling evidence for focal transcranial electrical brain stimulation in humans: A basis for high-definition tDCS. NeuroImage, 74:266-275.

Egner, $\mathrm{T}$.

2007. Congruency sequence effects and cognitive control. Cognitive, Affective, \& Behavioral Neuroscience, 7(4):380-390.

Egner, T. and J. Hirsch

2005a. Cognitive control mechanisms resolve conflict through cortical amplification of taskrelevant information. Nature Neuroscience, 8(12):1784-1790. 
Egner, T. and J. Hirsch

2005b. The neural correlates and functional integration of cognitive control in a Stroop task. NeuroImage, 24(2):539-547.

Emmerling, F., C. Martijn, H. J. E. M. Alberts, A. C. Thomson, B. David, D. Kessler, T. Schuhmann, and A. T. Sack

2017. The (non-)replicability of regulatory resource depletion: A field report employing noninvasive brain stimulation. PLOS ONE, 12(3):e0174331.

Eriksen, B. A. and C. W. Eriksen

1974. Effects of noise letters upon the identification of a target letter in a nonsearch task. Perception \& Psychophysics, 16(1):143-149.

Fell, J. and N. Axmacher

2011. The role of phase synchronization in memory processes. Nature Reviews Neuroscience, 12(2):105-118.

Fellows, L. K. and M. J. Farah

2005. Is anterior cingulate cortex necessary for cognitive control? Brain, 128(4):788-796.

Fertonani, A. and C. Miniussi

2017. Transcranial Electrical Stimulation. The Neuroscientist, 23(2):109-123.

Fries, $\mathrm{P}$.

2005. A mechanism for cognitive dynamics: neuronal communication through neuronal coherence. Trends in Cognitive Sciences, 9(10):474-480.

Fritz, J. and G. Dreisbach

2013. Conflicts as aversive signals: conflict priming increases negative judgments for neutral stimuli. Cognitive, affective \& behavioral neuroscience, 13(2):311-7.

Gabriel, M. and E. Orona

1982. Parallel and serial processes of the prefrontal and cingulate cortical systems during behavioral learning. Brain Research Bulletin, 8(6):781-785. 
Gelman, A., D. B. Rubin, and Others

1992. Inference from iterative simulation using multiple sequences. Statistical science, $7(4): 457-472$.

Gläscher, J., R. Adolphs, H. Damasio, A. Bechara, D. Rudrauf, M. Calamia, L. K. Paul, and D. Tranel

2012. Lesion mapping of cognitive control and value-based decision making in the prefrontal cortex. Proceedings of the National Academy of Sciences of the United States of America, 109(36):14681-6.

Goodale, M. A. and A. D. Milner

1992. Separate visual pathways for perception and action. Trends in neurosciences, 15(1):205.

Grant, D. A. and E. Berg 1948. A behavioral analysis of degree of reinforcement and ease of shifting to new responses in a Weigl-type card-sorting problem. Journal of Experimental Psychology, 38(4):404-411.

Gratton, G., M. G. Coles, and E. Donchin

1992. Optimizing the use of information: strategic control of activation of responses. Journal of experimental psychology. General, 121(4):480-506.

Greinacher, R., L. Buhôt, L. Möller, and G. Learmonth 2019. The time course of ineffective sham-blinding during low-intensity ( $1 \mathrm{~mA})$ transcranial direct current stimulation. European Journal of Neuroscience, 50(8):3380-3388.

Grossman, N., D. Bono, N. Dedic, S. B. Kodandaramaiah, A. Rudenko, H.-J. Suk, A. M. Cassara, E. Neufeld, N. Kuster, L.-H. Tsai, A. Pascual-Leone, and E. S. Boyden 2017. Noninvasive Deep Brain Stimulation via Temporally Interfering Electric Fields. Cell, 169(6):1029-1041.e16.

Guitart-Masip, M., R. Chowdhury, T. Sharot, P. Dayan, E. Duzel, and R. J. Dolan 2012. Action controls dopaminergic enhancement of reward representations. Proceedings of the National Academy of Sciences, 109(19):7511-7516. 
Guitart-Masip, M., E. Duzel, R. Dolan, and P. Dayan

2014. Action versus valence in decision making. Trends in Cognitive Sciences, 18(4):194202.

Haber, M., L. Zhou, and K. K. Murai

2006. Cooperative astrocyte and dendritic spine dynamics at hippocampal excitatory synapses. Journal of Neuroscience, 26(35):8881-8891.

Haber, S. N.

2016. Corticostriatal circuitry. Dialogues in clinical neuroscience, 18(1):7.

Hanes, K. R., D. G. Andrewes, D. J. Smith, and C. Pantelis

1996. A brief assessment of executive control dysfunction: discriminant validity and homogeneity of planning, set shift, and fluency measures. Archives of clinical neuropsychology : the official journal of the National Academy of Neuropsychologists, 11(3):185-91.

Hänninen, T., M. Hallikainen, K. Koivisto, K. Partanen, M. P. Laakso, P. J. Riekkinen, and H. Soininen

1997. Decline of frontal lobe functions in subjects with age-associated memory impairment. Neurology, 48(1):148-53.

Hanslmayr, S., B. Pastötter, K.-H. Bäuml, S. Gruber, M. Wimber, and W. Klimesch 2008. The electrophysiological dynamics of interference during the Stroop task. Journal of cognitive neuroscience, 20(2):215-25.

Heilbronner, S. R. and B. Y. Hayden

2016. Dorsal Anterior Cingulate Cortex: A Bottom-Up View. Annual Review of Neuroscience, 39(1):149-170.

Heise, K.-F., T. S. Monteiro, I. Leunissen, D. Mantini, and S. P. Swinnen

2019. Distinct online and offline effects of alpha and beta transcranial alternating current stimulation (tACS) on continuous bimanual performance and task-set switching. Scientific reports, 9(1):1-16.

Helfrich, R. F., T. R. Schneider, S. Rach, S. A. Trautmann-Lengsfeld, A. K. Engel, and C. S. 
Herrmann

2014. Entrainment of brain oscillations by transcranial alternating current stimulation. Current biology, 24(3):333-9.

Herrmann, C. S., S. Rach, T. Neuling, and D. Strüber

2013. Transcranial alternating current stimulation: a review of the underlying mechanisms and modulation of cognitive processes. Frontiers in human neuroscience, 7:279.

Hershberger, W. A.

1986. An approach through the looking-glass. Animal Learning \& Behavior, 14(4):443-451.

Hirsh, J. B., R. A. Mar, and J. B. Peterson

2012. Psychological entropy: a framework for understanding uncertainty-related anxiety. Psychological review, 119(2):304-20.

Holland, P. C.

1979. Differential effects of omission contingencies on various components of Pavlovian appetitive conditioned responding in rats. Journal of experimental psychology. Animal behavior processes, 5(2):178-93.

Holmes, P. and J. D. Cohen

2014. Optimality and Some of Its Discontents: Successes and Shortcomings of Existing Models for Binary Decisions. Topics in Cognitive Science, 6(2):258-278.

Holroyd, C. B., S. Nieuwenhuis, N. Yeung, L. Nystrom, R. B. Mars, M. G. H. Coles, and J. D. Cohen

2004. Dorsal anterior cingulate cortex shows fMRI response to internal and external error signals. Nature Neuroscience, 7(5):497-498.

Horvath, J. C., J. D. Forte, and O. Carter

2015. Evidence that transcranial direct current stimulation (tDCS) generates little-to-no reliable neurophysiologic effect beyond MEP amplitude modulation in healthy human subjects: A systematic review. Neuropsychologia, 66:213-236.

Hothorn, T., K. Hornik, and M. A. van de Wiel and Achim Zeileis 2006. A Lego System for Conditional Inference. The American Statistician, 60(3):257-263. 
Hothorn, T., K. Hornik, and M. A. van de Wiel and Achim Zeileis 2008. Implementing a Class of Permutation Tests: The coin Package. Journal of Statistical Software, 28(8):1-23.

Howson, C. and P. Urbach

1991. Bayesian reasoning in science. Nature, 350(6317):371-374.

Hsu, W.-Y., T. P. Zanto, M. R. van Schouwenburg, and A. Gazzaley

2017. Enhancement of multitasking performance and neural oscillations by transcranial alternating current stimulation. PloS one, 12(5).

Huang, Y.-Z., M.-K. Lu, A. Antal, J. Classen, M. Nitsche, U. Ziemann, M. Ridding, M. Hamada, Y. Ugawa, S. Jaberzadeh, A. Suppa, W. Paulus, and J. Rothwell

2017. Plasticity induced by non-invasive transcranial brain stimulation: A position paper. Clinical Neurophysiology, 128(11):2318-2329.

Inanaga, $\mathrm{K}$.

1998. Frontal midline theta rhythm and mental activity. Psychiatry and Clinical Neurosciences, 52(6):555-566.

Ishii, R., K. Shinosaki, S. Ukai, T. Inouye, T. Ishihara, T. Yoshimine, N. Hirabuki, H. Asada, T. Kihara, S. E. Robinson, and M. Takeda

1999. Medial prefrontal cortex generates frontal midline theta rhythm. NeuroReport, 10(4):675-679.

Ito, S.

2003. Performance Monitoring by the Anterior Cingulate Cortex During Saccade Countermanding. Science, 302(5642):120-122.

Jackson, A. F. and D. J. Bolger

2014. The neurophysiological bases of EEG and EEG measurement: A review for the rest of us. Psychophysiology, 51(11):1061-1071.

Jung, T.-P., C. Humphries, T.-W. Lee, S. Makeig, M. J. McKeown, V. Iragui, and T. J. Sejnowski 1998. Extended ICA removes artifacts from electroencephalographic recordings. In Advances in neural information processing systems, Pp. 894-900. 
Kahneman, D.

2011. Thinking, fast and slow. Macmillan.

Karabanov, A. N., G. B. Saturnino, A. Thielscher, and H. R. Siebner

2019. Can transcranial electrical stimulation localize brain function? Frontiers in psychology, $10: 213$.

Kasten, F. H., J. Dowsett, and C. S. Herrmann

2016. Sustained Aftereffect of $\alpha$-tACS Lasts Up to 70 min after Stimulation. Frontiers in human neuroscience, 10:245.

Kennerley, S. W., M. E. Walton, T. E. J. Behrens, M. J. Buckley, and M. F. S. Rushworth 2006. Optimal decision making and the anterior cingulate cortex. Nature Neuroscience, 9(7):940-947.

Kerns, J. G., J. D. Cohen, A. W. MacDonald, R. Y. Cho, V. A. Stenger, and C. S. Carter 2004. Anterior cingulate conflict monitoring and adjustments in control. Science (New York, N.Y.), 303(5660):1023-6.

Klein, G. S.

1964. Semantic power measured through the interference of words with color-naming. The American journal of psychology, 77:576-88.

Koga, S., R. Rothermel, C. Juhász, T. Nagasawa, S. Sood, and E. Asano

2011. Electrocorticographic correlates of cognitive control in a Stroop task-intracranial recording in epileptic patients. Human brain mapping, 32(10):1580-91.

Kohli, S. and A. J. Casson

2019. Removal of gross artifacts of transcranial alternating current stimulation in simultaneous eeg monitoring. Sensors, 19(1):190.

Kohli, S. and A. J. Casson

2020. Machine learning validation of EEG+ tACS artefact removal. Journal of neural engineering, 17(1):16034. 
Kool, W., J. T. McGuire, Z. B. Rosen, and M. M. Botvinick

2010. Decision making and the avoidance of cognitive demand. Journal of Experimental Psychology: General, 139(4):665-682.

Kuo, M.-F. and M. A. Nitsche

2012. Effects of Transcranial Electrical Stimulation on Cognition. Clinical EEG and Neuroscience, 43(3):192-199.

Kuznetsova, A., P. B. Brockhoff, and R. H. B. Christensen

2017. ImerTest Package: Tests in Linear Mixed Effects Models. Journal of Statistical Software, 82(13):1-26.

Laird, A. R., K. M. McMillan, J. L. Lancaster, P. Kochunov, P. E. Turkeltaub, J. V. Pardo, and P. T. Fox

2005. A comparison of label-based review and ALE meta-analysis in the Stroop task. Human Brain Mapping, 25(1):6-21.

Lefaucheur, J.-P., A. Antal, S. S. Ayache, D. H. Benninger, J. Brunelin, F. Cogiamanian, M. Cotelli, D. De Ridder, R. Ferrucci, B. Langguth, P. Marangolo, V. Mylius, M. A. Nitsche, F. Padberg, U. Palm, E. Poulet, A. Priori, S. Rossi, M. Schecklmann, S. Vanneste, U. Ziemann, L. Garcia-Larrea, and W. Paulus

2017. Evidence-based guidelines on the therapeutic use of transcranial direct current stimulation (tDCS). Clinical Neurophysiology, 128(1):56-92.

Lehr, A., N. Henneberg, T. Nigam, W. Paulus, and A. Antal

2019. Modulation of Conflict Processing by Theta-Range tACS over the Dorsolateral Prefrontal Cortex. Neural Plasticity, 2019:1-13.

Liotti, M., M. G. Woldorff, R. Perez, and H. S. Mayberg

2000. An ERP study of the temporal course of the Stroop color-word interference effect. Neuropsychologia, 38(5):701-11.

Liston, C., S. Matalon, T. A. Hare, M. C. Davidson, and B. Casey 2006. Anterior Cingulate and Posterior Parietal Cortices Are Sensitive to Dissociable Forms of Conflict in a Task-Switching Paradigm. Neuron, 50(4):643-653. 
Liu, A., M. Vöröslakos, G. Kronberg, S. Henin, M. R. Krause, Y. Huang, A. Opitz, A. Mehta, C. C. Pack, B. Krekelberg, and Others

2018. Immediate neurophysiological effects of transcranial electrical stimulation. Nature communications, 9(1):1-12.

Lo, S. and S. Andrews

2015. To transform or not to transform: using generalized linear mixed models to analyse reaction time data. Frontiers in Psychology, 6:1171.

Luk, C.-H. and J. D. Wallis

2013. Choice coding in frontal cortex during stimulus-guided or action-guided decisionmaking. The Journal of neuroscience : the official journal of the Society for Neuroscience, 33(5):1864-71.

MacDonald, A. W., J. D. Cohen, V. A. Stenger, and C. S. Carter

2000. Dissociating the role of the dorsolateral prefrontal and anterior cingulate cortex in cognitive control. Science (New York, N.Y.), 288(5472):1835-8.

MacLeod, C. M.

2005. The Stroop Task in Cognitive Research. In Cognitive methods and their applications to clinical research, Pp. 17-40.

MacLeod, C. M.

2015. The Stroop Effect. In Encyclopedia of Color Science and Technology, Pp. 1-6. Berlin, Heidelberg: Springer Berlin Heidelberg.

MacLeod, C. M. and P. A. MacDonald

2000. Interdimensional interference in the Stroop effect: uncovering the cognitive and neural anatomy of attention. Trends in Cognitive Sciences, 4(10):383-391.

Mansouri, F. A., M. J. Buckley, and K. Tanaka

2007. Mnemonic Function of the Dorsolateral Prefrontal Cortex in Conflict-Induced Behavioral Adjustment. Science, 318(5852):987-990.

Mansouri, F. A., K. Tanaka, and M. J. Buckley 
2009. Conflict-induced behavioural adjustment: a clue to the executive functions of the prefrontal cortex. Nature Reviews Neuroscience, 10(2):141-152.

McCulloch, C. E.

2003. Chapter 4: Generalized linear mixed models (GLMMs). In Generalized Linear Mixed Models, volume 7 of Regional Conference Series in Probability and Statistics, Pp. 28-33. Beechwood OH and Alexandria VA: Institute of Mathematical Statistics and American Statistical Association.

Medford, N. and H. D. Critchley

2010. Conjoint activity of anterior insular and anterior cingulate cortex: awareness and response. Brain structure \& function, 214(5-6):535-49.

Metzler-Baddeley, C., D. K. Jones, J. Steventon, L. Westacott, J. P. Aggleton, and M. J. O'Sullivan

2012. Cingulum microstructure predicts cognitive control in older age and mild cognitive impairment. The Journal of neuroscience : the official journal of the Society for Neuroscience, 32(49):17612-9.

Miller, B. L. and J. L. Cummings

2017. The human frontal lobes : functions and disorders, 3rd edition. Guildford Press.

Miller, E. K.

2000. The prefontral cortex and cognitive control. Nature Reviews Neuroscience, 1(1):59-65.

Miller, E. K. and J. D. Cohen

2001. An Integrative Theory of Prefrontal Cortex Function. Annual Review of Neuroscience, 24(1):167-202.

Minami, S. and K. Amano

2017. Illusory jitter perceived at the frequency of alpha oscillations. Current Biology, 27(15):2344-2351.

Miyake, A., N. P. Friedman, M. J. Emerson, A. H. Witzki, A. Howerter, and T. D. Wager 2000. The Unity and Diversity of Executive Functions and Their Contributions to Complex Frontal Lobe Tasks: A Latent Variable Analysis. Cognitive Psychology, 41(1):49-100. 
Morecraft, R. J. and G. W. Van Hoesen

1998. Convergence of limbic input to the cingulate motor cortex in the rhesus monkey. Brain research bulletin, 45(2):209-32.

Mtui, E., G. Gruener, P. Dockery, and M. J. T. FitzGerald

2015. Fitzgerald's clinical neuroanatomy and neuroscience.

Muthukumaraswamy, S. D., R. L. Carhart-Harris, R. J. Moran, M. J. Brookes, T. M. Williams, D. Errtizoe, B. Sessa, A. Papadopoulos, M. Bolstridge, K. D. Singh, A. Feilding, K. J. Friston, and D. J. Nutt

2013. Broadband cortical desynchronization underlies the human psychedelic state. The Journal of neuroscience : the official journal of the Society for Neuroscience, 33(38):1517183.

Nagel, I. E., E. H. Schumacher, R. Goebel, and M. D’Esposito 2008. Functional MRI investigation of verbal selection mechanisms in lateral prefrontal cortex. NeuroImage, 43(4):801-7.

Negahbani, E., F. H. Kasten, C. S. Herrmann, and F. Fröhlich

2018. Targeting alpha-band oscillations in a cortical model with amplitude-modulated highfrequency transcranial electric stimulation. NeuroImage, 173:3-12.

Nelder, J. A. and R. Mead

1965. A Simplex Method for Function Minimization. The Computer Journal, 7(4):308-313.

Niendam, T. A., A. R. Laird, K. L. Ray, Y. M. Dean, D. C. Glahn, and C. S. Carter

2012. Meta-analytic evidence for a superordinate cognitive control network subserving diverse executive functions. Cognitive, Affective, \& Behavioral Neuroscience, 12(2):241-268.

Nigbur, R., G. Ivanova, and B. Stürmer

2011. Theta power as a marker for cognitive interference. Clinical Neurophysiology, 122(11):2185-2194.

Niki, H. and M. Watanabe

1979. Prefrontal and cingulate unit activity during timing behavior in the monkey. Brain Research, 171(2):213-224. 
Nitsche, M. A. and W. Paulus

2000. Excitability changes induced in the human motor cortex by weak transcranial direct current stimulation. The Journal of physiology, 527 Pt 3:633-9.

Nyhus, E. and T. Curran

2010. Functional role of gamma and theta oscillations in episodic memory. Neuroscience \& Biobehavioral Reviews, 34(7):1023-1035.

Oehrn, C. R., S. Hanslmayr, J. Fell, L. Deuker, N. A. Kremers, A. T. Do Lam, C. E. Elger, and N. Axmacher

2014. Neural Communication Patterns Underlying Conflict Detection, Resolution, and Adaptation. Journal of Neuroscience, 34(31):10438-10452.

Oldfield, R.

1971. The assessment and analysis of handedness: The Edinburgh inventory. Neuropsychologia, 9(1):97-113.

Onoda, K., T. Kawagoe, H. Zheng, and S. Yamaguchi

2017. Theta band transcranial alternating current stimulations modulates network behavior of dorsal anterior cingulate cortex. Scientific Reports, 7(1):3607.

Oostenveld, R., P. Fries, E. Maris, and J. M. Schoffelen

2011. FieldTrip: Open source software for advanced analysis of MEG, EEG, and invasive electrophysiological data. Computational Intelligence and Neuroscience, 2011.

O’Reilly, R. C.

2010. The What and How of Prefrontal Cortical Organization. Trends in neurosciences, 33(8):355.

Pahor, A. and N. Jaušovec

2014. The effects of theta transcranial alternating current stimulation (tACS) on fluid intelligence. International Journal of Psychophysiology, 93(3):322-331.

Pallak, M. S., T. S. Pittman, J. F. Heller, and P. Munson

1975. The effect of arousal on Stroop color-word task performance. Bulletin of the Psychonomic Society, 6(3):248-250. 
Parkin, B., H. Ekhtiari, and V. Walsh

2015. Non-invasive Human Brain Stimulation in Cognitive Neuroscience: A Primer. Neuron, 87(5):932-945.

Parvizi, J., V. Rangarajan, W. R. Shirer, N. Desai, and M. D. Greicius

2013. The will to persevere induced by electrical stimulation of the human cingulate gyrus. Neuron, 80(6):1359-67.

Pasqualotto, A.

2016. Transcranial random noise stimulation benefits arithmetic skills. Neurobiology of Learning and Memory, 133:7-12.

Pastötter, B., G. Dreisbach, and K.-H. T. Bäuml

2013. Dynamic Adjustments of Cognitive Control: Oscillatory Correlates of the Conflict Adaptation Effect. Journal of Cognitive Neuroscience, 25(12):2167-2178.

Paulus, W.

2011. Transcranial electrical stimulation (tES tDCS; tRNS, tACS) methods. Neuropsychological Rehabilitation, 21(5):602-617.

Paus, T.

2001. Primate anterior cingulate cortex: Where motor control, drive and cognition interface. Nature Reviews Neuroscience, 2(6):417-424.

Paus, T., L. Koski, Z. Caramanos, and C. Westbury

1998. Regional differences in the effects of task difficulty and motor output on blood flow response in the human anterior cingulate cortex. NeuroReport, 9(9):R37-R47.

Peirce, J. W.

2007. PsychoPy-Psychophysics software in Python. Journal of neuroscience methods, 162(12):8-13.

Peirce, J. W.

2009. Generating stimuli for neuroscience using PsychoPy. Frontiers in neuroinformatics, $2: 10$. 
Peters, J. and C. Büchel

2010. Episodic Future Thinking Reduces Reward Delay Discounting through an Enhancement of Prefrontal-Mediotemporal Interactions. Neuron, 66(1):138-148.

Polanía, R., M. A. Nitsche, and C. C. Ruff

2018. Studying and modifying brain function with non-invasive brain stimulation. Nature Neuroscience, 21(2):174-187.

Premack, D.

2007. Human and animal cognition: continuity and discontinuity. Proceedings of the National Academy of Sciences of the United States of America, 104(35):13861-7.

Quintana, J. and J. M. Fuster

1999. From Perception to Action: Temporal Integrative Functions of Prefrontal and Parietal Neurons. Cerebral Cortex, 9(3):213-221.

R Core Team

2018. R: A Language and Environment for Statistical Computing. R Foundation for Statistical Computing, Vienna, Austria.

Ratcliff, R.

1978. A theory of memory retrieval. Psychological Review, 85(2):59-108.

Ratcliff, R. and G. McKoon

2008. The diffusion decision model: theory and data for two-choice decision tasks. Neural computation, 20(4):873-922.

Redish, A. D.

2016. Vicarious trial and error. Nature Reviews Neuroscience, 17(3):147.

Reed, T. and R. C. Kadosh

2018. Transcranial electrical stimulation (tES) mechanisms and its effects on cortical excitability and connectivity. Journal of inherited metabolic disease, 41(6):1123-1130.

Rossini, P., D. Burke, R. Chen, L. Cohen, Z. Daskalakis, R. Di Iorio, V. Di Lazzaro, F. Ferreri, P. Fitzgerald, M. George, M. Hallett, J. Lefaucheur, B. Langguth, H. Matsumoto, C. Miniussi, 
M. Nitsche, A. Pascual-Leone, W. Paulus, S. Rossi, J. Rothwell, H. Siebner, Y. Ugawa, V. Walsh, and U. Ziemann

2015. Non-invasive electrical and magnetic stimulation of the brain, spinal cord, roots and peripheral nerves: Basic principles and procedures for routine clinical and research application. An updated report from an I.F.C.N. Committee. Clinical Neurophysiology, 126(6):1071-1107.

Rushworth, M., M. Noonan, E. Boorman, M. Walton, and T. Behrens

2011. Frontal Cortex and Reward-Guided Learning and Decision-Making. Neuron, 70(6):1054-1069.

Rushworth, M. F. S., K. A. Hadland, D. Gaffan, and R. E. Passingham 2003. The Effect of Cingulate Cortex Lesions on Task Switching and Working Memory. Journal of Cognitive Neuroscience, 15(3):338-353.

Saturnino, G. B., A. Antunes, and A. Thielscher

2015. On the importance of electrode parameters for shaping electric field patterns generated by tDCS. NeuroImage, 120:25-35.

Schnitzler, A. and J. Gross

2005. Normal and pathological oscillatory communication in the brain. Nature Reviews Neuroscience, 6(4):285-296.

Schoen, I. and P. Fromherz

2008. Extracellular stimulation of mammalian neurons through repetitive activation of $\mathrm{Na}+$ channels by weak capacitive currents on a silicon chip. Journal of neurophysiology, 100(1):346-357.

Schutter, D. J.

2014. Syncing your brain: electric currents to enhance cognition. Trends in Cognitive Sciences, 18(7):331-333.

Sela, T., A. Kilim, and M. Lavidor

2012. Transcranial Alternating Current Stimulation Increases Risk-Taking Behavior in the Balloon Analog Risk Task. Frontiers in Neuroscience, 6:22. 
Shackman, A. J., T. V. Salomons, H. A. Slagter, A. S. Fox, J. J. Winter, and R. J. Davidson 2011. The integration of negative affect, pain and cognitive control in the cingulate cortex. Nature reviews. Neuroscience, 12(3):154-67.

Shenhav, A., M. Botvinick, and J. Cohen

2013. The Expected Value of Control: An Integrative Theory of Anterior Cingulate Cortex Function. Neuron, 79(2):217-240.

Shenhav, A., S. Musslick, F. Lieder, W. Kool, T. L. Griffiths, J. D. Cohen, and M. M. Botvinick 2017. Toward a rational and mechanistic account of mental effort. Annual review of neuroscience, 40:99-124.

Sheth, S. A., M. K. Mian, S. R. Patel, W. F. Asaad, Z. M. Williams, D. D. Dougherty, G. Bush, and E. N. Eskandar

2012. Human dorsal anterior cingulate cortex neurons mediate ongoing behavioural adaptation. Nature, 488(7410):218-221.

Shidara, M. and B. J. Richmond

2002. Anterior Cingulate: Single Neuronal Signals Related to Degree of Reward Expectancy. Science, 296(5573):1709-1711.

Silton, R. L., W. Heller, D. N. Towers, A. S. Engels, J. M. Spielberg, J. C. Edgar, S. M. Sass, J. L. Stewart, B. P. Sutton, M. T. Banich, and G. A. Miller 2010. The time course of activity in dorsolateral prefrontal cortex and anterior cingulate cortex during top-down attentional control. NeuroImage, 50(3):1292-1302.

Simon, J. R. and A. P. Rudell

1967. Auditory S-R compatibility: The effect of an irrelevant cue on information processing. Journal of Applied Psychology, 51(3):300-304.

Singer, W. and C. M. Gray

1995. Visual Feature Integration and the Temporal Correlation Hypothesis. Annual Review of Neuroscience, 18(1):555-586.

Smith, E. H., G. P. Banks, C. B. Mikell, S. S. Cash, S. R. Patel, E. N. Eskandar, and S. A. Sheth 2015. Frequency-Dependent Representation of Reinforcement-Related Information in the Hu- 
man Medial and Lateral Prefrontal Cortex. The Journal of neuroscience : the official journal of the Society for Neuroscience, 35(48):15827-36.

Smith, E. H., G. Horga, M. J. Yates, C. B. Mikell, G. P. Banks, Y. J. Pathak, C. A. Schevon, G. M. McKhann, B. Y. Hayden, M. M. Botvinick, and Others 2019. Widespread temporal coding of cognitive control in the human prefrontal cortex. Nature neuroscience, Pp. 1-9.

Smittenaar, P., T. H. B. FitzGerald, V. Romei, N. D. Wright, and R. J. Dolan 2013. Disruption of dorsolateral prefrontal cortex decreases model-based in favor of modelfree control in humans. Neuron, 80(4):914-919.

Sridharan, D., D. J. Levitin, and V. Menon

2008. A critical role for the right fronto-insular cortex in switching between centralexecutive and default-mode networks. Proceedings of the National Academy of Sciences, 105(34):12569-12574.

Stacey, W. C. and D. M. Durand

2000. Stochastic resonance improves signal detection in hippocampal CA1 neurons. Journal of Neurophysiology, 83(3):1394-1402.

Stafford, T., L. Ingram, and K. N. Gurney

2011. Piéron's Law Holds During Stroop Conflict: Insights Into the Architecture of Decision Making. Cognitive Science, 35(8):1553-1566.

Stan development, T.

2016. RStan: the R interface to Stan. R package version, 2(1).

Strauss, E., E. M. S. Sherman, O. Spreen, and O. Spreen

2006. A compendium of neuropsychological tests : administration, norms, and commentary. Oxford University Press.

Stroop, J. R.

1935. Studies of interference in serial verbal reactions. Journal of Experimental Psychology, 18(6):643-662. 
Stuss, D. T.

2011. Functions of the Frontal Lobes: Relation to Executive Functions. Journal of the International Neuropsychological Society, 17(05):759-765.

Stuss, D. T. and M. P. Alexander

2007. Is there a dysexecutive syndrome? Philosophical transactions of the Royal Society of London. Series B, Biological sciences, 362(1481):901-15.

Tang, D., L. Hu, and A. Chen

2013. The neural oscillations of conflict adaptation in the human frontal region. Biological Psychology, 93(3):364-372.

Team, R. and Others

2015. RStudio: integrated development for R. RStudio. Inc., Boston, MA, 639:640.

Terney, D., L. Chaieb, V. Moliadze, A. Antal, and W. Paulus

2008. Increasing Human Brain Excitability by Transcranial High-Frequency Random Noise Stimulation. Journal of Neuroscience, 28(52):14147-14155.

Thielscher, A., A. Antunes, and G. B. Saturnino

2015. Field modeling for transcranial magnetic stimulation: A useful tool to understand the physiological effects of TMS? In 2015 37th Annual International Conference of the IEEE Engineering in Medicine and Biology Society (EMBC), volume 2015, Pp. 222-225. IEEE.

To, W. T., J. Eroh, J. Hart, and S. Vanneste

2018. Exploring the effects of anodal and cathodal high definition transcranial direct current stimulation targeting the dorsal anterior cingulate cortex. Scientific Reports, 8(1):4454.

Turi, Z., G. G. Ambrus, K. Janacsek, K. Emmert, L. Hahn, W. Paulus, and A. Antal 2013. Both the cutaneous sensation and phosphene perception are modulated in a frequencyspecific manner during transcranial alternating current stimulation. Restorative neurology and neuroscience, 31(3):275-285.

Turi, Z., G. Csifcsák, N. M. Boayue, P. Aslaksen, A. Antal, W. Paulus, J. Groot, G. E. Hawkins, 
B. Forstmann, A. Opitz, and Others

2019. Blinding is compromised for transcranial direct current stimulation at $1 \mathrm{~mA}$ for $20 \mathrm{~min}$ in young healthy adults. European Journal of Neuroscience, 50(8):3261-3268.

Uhlhaas, P. J. and W. Singer

2013. High-frequency oscillations and the neurobiology of schizophrenia. Dialogues in clinical neuroscience, 15(3):301.

Ullsperger, M., H. A. Harsay, J. R. Wessel, and K. R. Ridderinkhof

2010. Conscious perception of errors and its relation to the anterior insula. Brain structure \& function, 214(5-6):629-43.

Ulrich, R., H. Schröter, H. Leuthold, and T. Birngruber

2015. Automatic and controlled stimulus processing in conflict tasks: Superimposed diffusion processes and delta functions. Cognitive Psychology, 78:148-174.

van Driel, J., I. G. Sligte, J. Linders, D. Elport, and M. X. Cohen

2015. Frequency Band-Specific Electrical Brain Stimulation Modulates Cognitive Control Processes. PloS one, 10(9):e0138984.

van Veen, V., J. D. Cohen, M. M. Botvinick, V. Stenger, and C. S. Carter

2001. Anterior Cingulate Cortex, Conflict Monitoring, and Levels of Processing. NeuroImage, 14(6):1302-1308.

Vehtari, A., A. Gelman, and J. Gabry

2017. Practical Bayesian model evaluation using leave-one-out cross-validation and WAIC. Statistics and computing, 27(5):1413-1432.

Veniero, D., C. S. Y. Benwell, M. M. Ahrens, and G. Thut

2017. Inconsistent Effects of Parietal $\alpha$-tACS on Pseudoneglect across Two Experiments: A Failed Internal Replication. Frontiers in psychology, 8:952.

Venkatraman, V. and S. A. Huettel

2012. Strategic control in decision-making under uncertainty. European Journal of Neuroscience, 35(7):1075-1082. 
Villamar, M. F., M. S. Volz, M. Bikson, A. Datta, A. F. Dasilva, and F. Fregni 2013. Technique and considerations in the use of $4 \times 1$ ring high-definition transcranial direct current stimulation (HD-tDCS). Journal of visualized experiments : JoVE, (77):e50309.

Vogt, B. A. and M. Gabriel, eds.

1993. Neurobiology of Cingulate Cortex and Limbic Thalamus. Boston, MA: Birkhäuser Boston.

Vogt, B. A., L. Vogt, N. B. Farber, and G. Bush

2005. Architecture and neurocytology of monkey cingulate gyrus. The Journal of Comparative Neurology, 485(3):218-239.

Vöröslakos, M., Y. Takeuchi, K. Brinyiczki, T. Zombori, A. Oliva, A. Fernández-Ruiz, G. Kozák, Z. T. Kincses, B. Iványi, G. Buzsáki, and A. Berényi

2018. Direct effects of transcranial electric stimulation on brain circuits in rats and humans. Nature Communications, 9(1):483.

Voytek, B. and R. T. Knight

2015. Dynamic Network Communication as a Unifying Neural Basis for Cognition, Development, Aging, and Disease. Biological Psychiatry, 77(12):1089-1097.

Wagenmakers, E.-J. and S. Farrell

2004. AIC model selection using Akaike weights. Psychonomic bulletin \& review, 11(1):192196.

West, R. and C. Alain

1999. Event-related neural activity associated with the Stroop task. Cognitive Brain Research, $8(2): 157-164$.

West, R., K. Jakubek, N. Wymbs, M. Perry, and K. Moore 2005. Neural correlates of conflict processing. Experimental brain research, 167(1):38-48.

Westbrook, A., D. Kester, and T. S. Braver

2013. What Is the Subjective Cost of Cognitive Effort? Load, Trait, and Aging Effects Revealed by Economic Preference. PLoS ONE, 8(7):e68210. 
White, C. N., M. Servant, and G. D. Logan

2018. Testing the validity of conflict drift-diffusion models for use in estimating cognitive processes: A parameter-recovery study. Psychonomic Bulletin \& Review, 25(1):286-301.

Wunderlich, K., P. Dayan, and R. J. Dolan

2012. Mapping value based planning and extensively trained choice in the human brain. Nature neuroscience, 15(5):786.

Yao, Y., A. Vehtari, D. Simpson, A. Gelman, and Others 2018. Using stacking to average Bayesian predictive distributions (with discussion). Bayesian Analysis, 13(3):917-1007.

Yavari, F., A. Jamil, M. Mosayebi Samani, L. P. Vidor, and M. A. Nitsche 2018. Basic and functional effects of transcranial Electrical Stimulation (tES)An introduction. Neuroscience \& Biobehavioral Reviews, 85:81-92.

Yeung, N., M. M. Botvinick, and J. D. Cohen 2004. The Neural Basis of Error Detection: Conflict Monitoring and the Error-Related Negativity. Psychological Review, 111(4):931-959. 


\section{List of Figures}

1 Regions of cognitive control network . . . . . . . . . . . . . . 3

2 Anatomy and functions of DLPFC and dACC ............ 5

3 Conflict adaptation effect . . . . . . . . . . . . . . 7

4 Stroop task during DLPFC stimulation . . . . . . . . . . . . . . . 25

$5 \quad$ Electric field over DLPFC . . . . . . . . . . . . . . 26

6 DLPFC stimulation affects response times . . . . . . . . . . . . 35

$7 \quad$ DMC parameter recovery . . . . . . . . . . . . . . 43

8 Experimental procedure including stimulation and EEG . . . . . . . . . . 51

9 Electric field over dACC . . . . . . . . . . . . . . 52

10 Region of interest in time-frequency analysis . . . . . . . . . . . 55

$116 \mathrm{~Hz}$ stimulation to dACC affects Stroop effect . . . . . . . . . . 58

12 Stroop-like theta power difference itemized by stimulation . . . . . . . . . 62

13 Influence of stimulations on theta power differences $\ldots \ldots \ldots$

14 Experimental procedure during learning and transfer phase . . . . . . . 75

15 Stimulation montages, protocols and simulations . . . . . . . . . 76

16 Posterior predictive checks for logistic regression model . . . . . . . . . 82

17 Accuracy levels of card types between sessions . . . . . . . . . . . . 84

18 Accuracy and learning rate compared between sessions . . . . . . . . . 85

19 Model predictions misfit observed data . . . . . . . . . . . . . . . 90

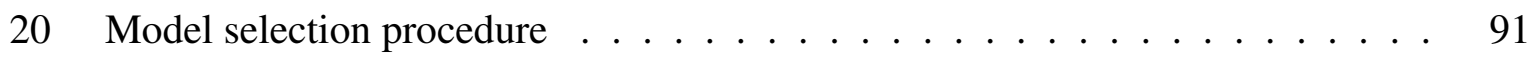

21 Probabilities of Go-responses . . . . . . . . . . . . . . . . 91

22 All individual responses . . . . . . . . . . . . . . . . . . . 92

23 Comparison of average accuracies . . . . . . . . . . . . . . 92

24 Comparison of learning rates . . . . . . . . . . . . . . . . 93

25 Perceptual adverse effects during tACS . . . . . . . . . . . . . . 93

26 The subjective ranking of the cards . . . . . . . . . . . . . . 95 


\section{List of Tables}

1 Accuracy and response times of experiment 1 and $2 \ldots \ldots 29$

2 Statistics - accuracy and response times of experiment $1 \ldots \ldots 32$

3 Statistics - accuracy and response times of experiment 2 . . . . . . . . 34

4 Statistics - accuracy and response times of experiments combined . . . . . 37

5 Demographics of theta tACS over DLPFC . . . . . . . . . . . . . . . 44

6 Arousal and sleep of theta tACS over DLPFC . . . . . . . . . . . . . 45

7 Accuracy and response times of stimulation phase . . . . . . . . . . 57

8 Statistics - response times of stimulation phase f . . . . . . . . . . 59

9 Accuracy and response times of before and after stimulation phase . . . . . 60

10 Statistics - response times before and after stimulation . . . . . . . . . . . 61

11 Statistics - non-phase locked theta power . . . . . . . . . . . . 67

12 Statistics - response times of stimulation phase . . . . . . . . . 81 


\section{A ACKNOWLEDGMENTS}

\section{A Acknowledgments}

I am very grateful to Prof. Andrea Antal for giving me the opportunity to work on this project and for her support throughout the last three and a half years. I also owe my gratitude to my direct collaborators Tarana Nigam, Iris Steinmann, Micha Siegle, Niklas Henneberg and Zsolt Turi who contributed majorly to all projects during the past three and a half years.

Furthermore, I want to thank all my colleagues from the department of Clinical Neurophysiology for instructions, comments, support and their collegiality.

This dissertation marks an important milestone for the educational training that began in Göttingen in 2014. I also want to express my gratitude to the staff of the IMPRS for Neurosciences for accepting me into this distinguished program and for the academic and financial support. Expressed officially but with the same gratitude: This work was partly supported by the Ph.D. program "Neurosciences" - International Max Planck Research School at the Georg August University Göttingen.

Lastly and most importantly, I want to thank my friends and family for their support and guidance. My friends in Göttingen and my SO have turned this city into a great home in the past six years. 


\section{B Declaration of Contribution}

I hereby declare that I wrote this thesis independently and with no other sources and aids than quoted.

Albert Lehr

Göttingen, 2nd of April, 2020 


\section{Curriculum Vitae}

\section{Albert Lehr}

\section{Personal Information}

E-Mail_ alehrresearch@gmail.com

Nationality German

Date / place of birth 23. May 1992 / Berlin, Germany

\section{Education}

2016 - present Dr. rer. nat., University of Göttingen, Germany

Dissertation: "Modulation of neuronal excitability in the cognitive control network by electrical stimulation"

Supervisor: Prof. Andrea Antal

2014 - 2016 Master's degree of Neuroscience, University of Göttingen, Germany

Master thesis: "Developing no report readouts for conscious visibility"

Supervisor: Prof. Melanie Wilke

2011 - 2014 Bachelor's degree of Molecular Biomedicine, University of Bonn,

Germany

Bachelor thesis: "The influence of the desmin cytoskeleton on the contractility of murine cardiomyocytes"

Supervisor: Prof. Rainer Meyer

\section{Publications}

de Lara, G. A., I. Alekseichuk, Z. Turi, A. Lehr, A. Antal, and W. Paulus

2018. Perturbation of theta-gamma coupling at the temporal lobe hinders verbal declarative memory. Brain stimulation, 11(3):509-517. 
Lehr, A., N. Henneberg, T. Nigam, W. Paulus, and A. Antal

2019. Modulation of Conflict Processing by Theta-Range tACS over the Dorsolateral Prefrontal Cortex. Neural Plasticity, 2019:1-13.

Turi, Z., M. Mittner, A. Lehr, H. Bürger, A. Antal, and W. Paulus

Theta-gamma cross-frequency transcranial alternating current stimulation over the trough impairs cognitive control. Submitted to eNeuro, doi: 10.31234/osf.io/4y3uh.

Lehr, A., M. Siegle, W. Paulus, and A. Antal

Theta transcranial alternating current stimulation decreases conflict effect in Stroop task. In Preparation.

\section{$\underline{\text { Poster presentation }}$}

July 2018 FENS, Forum 2018, Berlin, Germany

\section{Talk}

December 2018 Multi-centre Initiative, Transcranial Brain Stimulation in Cognitive Neuroscience Workshop, Rovereto, Italy

"Theta-gamma phase-coupling stimulation improves spatial working memory"

\section{$\underline{\text { Scientific volunteering }}$}

2018 - 2019 Ph.D. representative of IMPRS for Neurosciences

May 2018 8th Neuroscience Conference Neurizons, Göttingen, Germany 


\section{$\underline{\text { Scholarships }}$}

2014 - 2015 Stipend by the International Max Planck Research School Neurosciences, Göttingen

2014 RISE weltweit scholarship, Deutscher Akademischer Auslandsdienst, Germany

2013 RISE weltweit scholarship, Deutscher Akademischer Auslandsdienst, Germany

\section{$\underline{\text { Software experience }}$}

Programming in R, Matlab, Python

Inkscape for vector design

Lyx (knitr), Latex, Word for typesetting 